\title{
Musculoskeletal modelling of an ostrich (Struthio camelus) pelvic limb: Influence of limb orientation on muscular capacity during locomotion
}

John R Hutchinson, Jeffery W Rankin, Jonas Rubenson, Kate H Rosenbluth, Robert A Siston, Scott L Delp

We developed a three-dimensional, biomechanical computer model of the 36 major pelvic limb muscle groups in an ostrich (Struthio camelus) to investigate muscle function in this, the largest of extant birds and model organism for many studies of locomotor mechanics, body size, anatomy and evolution. Combined with experimental data, we use this model to test two main hypotheses. We first query whether ostriches use limb orientations (joint angles) that optimize the moment-generating capacities of their muscles during walking or running. Next, we test whether ostriches use limb orientations at mid-stance that keep their extensor muscles near maximal, and flexor muscles near minimal, moment arms. Our two hypotheses relate to the control priorities that a large bipedal animal might evolve under biomechanical constraints to achieve more effective static weight support. We find that ostriches do not use limb orientations to optimize the moment-generating capacities or moment arms of their muscles. We infer that dynamic properties of muscles or tendons might be better candidates for locomotor optimization. Regardless, general principles explaining why species choose particular joint orientations during locomotion are lacking, raising the question of whether such general principles exist or if clades evolve different patterns (e.g. weighting of muscle force-length or force-velocity properties in selecting postures). This leaves theoretical studies of muscle moment arms estimated for extinct animals at an impasse until studies of extant taxa answer these questions. Finally, we compare our model's results against those of two prior studies of ostrich limb muscle moment arms, finding general agreement for many muscles. Some flexor and extensor muscles exhibit self-stabilization patterns (posture-dependent switches between flexor/extensor action) that ostriches may use to coordinate their locomotion. However, some conspicuous areas of disagreement in our results illustrate some cautionary principles. Importantly, tendon-travel empirical measurements of muscle moment arms must be carefully designed to preserve 3D muscle geometry lest their accuracy suffer relative to that of anatomically realistic models. The dearth of accurate experimental measurements of 3D moment arms of muscles in birds leaves uncertainty regarding the relative accuracy of different modelling or experimental datasets such as in ostriches. Our 
model, however, provides a comprehensive set of 3D estimates of muscle actions in ostriches for the first time, emphasizing that avian limb mechanics are highly threedimensional and complex, and how no muscles act purely in the sagittal plane. A comparative synthesis of experiments and models such as ours could provide powerful synthesis into how anatomy, mechanics and control interact during locomotion and how these interactions evolve. Such a framework could remove obstacles impeding the analysis of muscle function in extinct taxa. 


\section{Musculoskeletal modelling of an ostrich (Struthio camelus) pelvic limb: Influence of limb} orientation on muscular capacity during locomotion

John R. Hutchinson ${ }^{1,2}$, Jeffery W. Rankin ${ }^{1}$, Jonas Rubenson ${ }^{3,4}$, Kate H. Rosenbluth ${ }^{2}$, Robert A. Siston $^{2,5}$, Scott L. Delp ${ }^{2}$

${ }^{1}$ Structure and Motion Laboratory, Department of Comparative Biomedical Sciences, The Royal Veterinary College, University of London, Hatfield, Hertfordshire, AL9 7TA, United Kingdom. ${ }^{2}$ Bioengineering Department, 318 Campus Drive, Stanford University, Stanford, California, 94305, USA.

${ }^{3}$ School of Sport Science, Exercise and Health, The University of Western Australia, Perth, WA, 6009, Australia.

${ }^{4}$ Department of Kinesiology, 29B Recreation Building, The Pennsylvania State University, University Park, PA, 16802, USA.

${ }^{5}$ Department of Mechanical and Aerospace Engineering, The Ohio State University, Columbus, $\mathrm{OH}, 43210$, USA.

Phone: +44-1707-666-313; Fax: +44-1707-666-371; email jhutchinson@rvc.ac.uk

Revised manuscript for PeerJ; www.peerj.org 


\section{Introduction}

As the largest living avian bipeds, ostriches (Struthio camelus Linnaeus 1758) are important for understanding how body mass influences locomotor mechanics in birds. In addition, ostriches are among the fastest of living terrestrial animals, and are the fastest living (perhaps even the fastest ever) bipedal runners. These birds can reach maximum speeds $>15 \mathrm{~ms}^{-1}$ (Alexander et al., 1979); similar to another biped that is coincidentally of similar size: red kangaroos (Macropus rufus) (Bennett \& Taylor, 1995). Examination of their locomotor dynamics may reveal some of the complex factors that determine maximum running speed in land animals and guide the development of fast running machines. Ostriches are also of similar body size to humans, which other than birds are the only obligate striding bipeds today, making comparisons of bipedal locomotor function in these two species possible (e.g., Gatesy and Biewener, 1991; Rubenson et al., 2011). Additionally, as the largest extant birds, ostriches are important "endpoints" for studies of body size effects on locomotion (e.g., Maloiy et al., 1979; Gatesy et al., 2009; Brassey et al., 2013a,b; Kilbourne, 2013). Furthermore, ostriches are members of the ratite bird clade, whose evolution from basal flying birds into large cursorial flightless animals has been of longstanding scientific interest. However, the evolutionary patterns and processes that produced the diversity of living ratites and their unusual locomotor mechanisms remain uncertain (Baker et al., 2014 and references therein). In turn, ratite birds including ostriches occupy relatively basal positions in extant avian phylogeny (e.g., Clarke and Cracraft, 2001; Baker et al., 2014). Despite their remarkable cursorial specializations and evolutionarily increased body size, ostriches can offer clues to the structure and function of earliest crown clade birds, and thereby about the evolution of avian locomotion from their theropod dinosaur forebears (e.g., Gatesy, 1990). Ostriches have also often been employed as analogues for dinosaur locomotion (e.g., Russell, 1972; Paul, 1998) despite some major anatomical differences (Gatesy, 1990,1995; Hutchinson and Gatesy, 2000; Hutchinson, 2002; Gatesy et al., 2009; Hutchinson \& Allen, 2009), so ostriches and extinct dinosaurs may be reciprocally informative.

A general problem facing those interested in examining the above questions in detail is that ostrich locomotion, including pelvic limb structure-function relationships during movement, remains incompletely understood. Numerous studies have empirically investigated the locomotor kinematics and kinetics of ostriches (e.g., Alexander et al., 1979; Gatesy and Biewener, 1991; Abourachid, 2001; Abourachid and Renous, 2000; Rubenson et al., 2004,2007,2011, Smith et al., 2006,2007,2010,2013; Jindrich et al., 2007; Schaller et al., 2009,2011) and much focus has been given to the whole-body mechanics and energetics of ostriches (e.g., Fedak and Seeherman, 1979, Fedak et al., 1982; Rubenson et al., 2004, Watson et al., 2011). However, detailed understanding of the interactions between the various components of the musculoskeletal system and the environment during these movements remain poorly understood. Due to the complex, non-linear dynamics of the musculoskeletal system, correlating whole-body level measures of locomotion to specific muscle function-structure relationships cannot yet be adequately performed.

Furthermore, ostrich myology was not carefully described until recently (Weissengruber et al., 2003; Gangl et al., 2004; Zinoviev, 2006; also knee joint functional morphology by Fuss, 1996; foot/ankle function by Schaller et al., 2009,2011). Previous myological studies were marred by errors in interpreting ostrich anatomy and by confusing application of mammalian anatomy to ostriches (e.g., Haughton, 1864; Macalister, 1864) or provided only a superficial 
treatment of proximal pelvic limb anatomy (e.g., Mellett, 1994). Even Gadow (1880), a classic comparative reference, contains antiquated concepts of homology (Rowe, 1986). These prior studies of ostrich myology augment comparative work on the pelvic limb myology of other ratites (e.g., Haughton, 1867a,b; Gadow, 1880; McGowan, 1979; Vanden Berge, 1982; Patak and Baldwin, 1998; Picasso 2010,2012; Chadwick et al., 2014; Lamas et al., 2014; Regnault et al., 2014). Additionally, data are available on muscle physiology in ostriches (Velotto and Crasto, 2004) and other ratites (e.g., McGowan, 1979; Patak and Baldwin, 1993), although biomechanical data characterizing muscle force-velocity and force-length relationships for avian pelvic limb muscles are scant (e.g., Nelson et al., 2004).

This body of prior research provides the strong foundation necessary for detailed examination of ostrich limb muscle function using anatomically-realistic biomechanical modelling in order to advance understanding of how the largest living bird supports its body weight and moves itself with its pelvic limb muscles. Here, to provide new insight into ostrich locomotor structure-function relationships, we investigate how pelvic limb muscle functions relate to limb orientation (i.e., posture/pose or joint angles; Gatesy, 1995; Gatesy et al., 2009) in ostriches. To do this, we integrate data from experimentally measured joint kinematics and ground reaction forces with a biomechanical computer model that was constructed by digitizing the bones, muscles, and tendons of an adult ostrich. By replicating ostrich structure and behaviour, the musculoskeletal model provides estimates of individual muscle moment arms (Pandy, 1999) and maximum capacity for moment generation during those behaviours: quantities that are otherwise difficult or impossible to accurately measure non-invasively, especially for all thirty-six major pelvic limb muscles simultaneously. Here we use this model to address some fundamental mysteries about locomotion in ostriches as well as birds, non-avian dinosaurs and bipeds.

Larger mammalian species tend to have straighter limbs than smaller species to improve their effective mechanical advantage during movement (Biewener, 1989, 1990). Yet it remains unclear how much of this improvement is achieved by reducing the moment arms of ground reaction forces about the limb joints using less flexed limb joint orientations or by increasing muscle moment arms via increased anatomical leverage (e.g., relatively larger trochanters) or straightened limb orientation (i.e., which shifts muscles further away from joint centres; Hutchinson et al., 2005). Our ostrich musculoskeletal model will facilitate discerning this relationship in birds, for whom it seems a similar pattern to mammals of having improved mechanical advantage in larger species holds (Gatesy and Biewener, 1991; Hutchinson, 2004; Günther et al., 2004; Brassey et al., 2013a; Kilbourne et al., 2013).

Previous studies (e.g., Hutchinson et al., 2005; and references therein) suggested that limb antigravity muscle moment arms (or moment-generating capacity; Full and Ahn, 1995) may peak in very upright limb orientations, which intimates that smaller animals with more crouched poses (e.g., birds) employ sub-optimal joint angles for supporting their body weight (Biewener, 1989; Günther et al., 2004), presumably as a tradeoff to provide other benefits such as increased manoeuvrability (e.g., Daley and Usherwood, 2010). As the largest living birds, ostriches stand and move with straighter limbs than smaller birds (Gatesy and Biewener, 1991). However ostriches are bipeds that still habitually support themselves with markedly flexed hip and knee joints, which make them a useful case study of this mechanical relationship between posture and antigravity muscle capacity. Understanding this relationship impacts the broader question of why animals choose certain postures - do they select postures that favour larger moment arms for economical force production (e.g., Fujiwara, 2009; Fujiwara et al., 2011; Fujiwara and 
122 Hutchinson, 2012) or other factors such as muscle force-length properties (e.g., McClearn, 1985; 123 Lieber \& Boakes, 1988a,b; Lieber \& Brown, 1992; Lieber \& Shoemaker, 1992; Lieber, 1997)?

The moment arms of ostrich pelvic limb muscles have been studied before with two different methodologies: two-dimensional (2D) experimental ("tendon travel") measurements of de-fleshed limbs (Smith et al., 2007) and 3D musculoskeletal computer models constructed from literature data, dissections and scanned skeletons (Bates and Schachner, 2012). The question remains open, how accurate are these experimental measurements and models, and how consistently can different researchers construct such models given the inherent subjectivity involved? This methodological question impacts many of the questions above; a weak model impairs the ability to test hypotheses. Here, we use our 3D musculoskeletal model and previously collected experimental data to address three main questions related to the issues described above: (1) Do ostriches adopt limb orientations during walking or running that optimize their capacity to generate maximal moments about the pelvic limb joints? (2) Are the moment arms of limb muscles maximized (for antigravity/extensor muscles; or minimized in the case of antagonistic flexor muscles) at mid-stance of locomotion (optimizing weight support), or at highly extended limb orientations, as prior studies of dinosaurs inferred (e.g., Hutchinson et al., 2005)? Finally, (3) how accurate or repeatable are estimates of limb muscle moment arms in ostriches using different methods?

We also integrate our results with previous studies of ostriches and other large birds

\section{Materials and Methods}

\section{Animals}

Experimental biomechanics data were collected from three female adult ostriches (70.0, $78.7,75.9 \mathrm{~kg}$ body mass), from which a single representative animal's data was used as model input $(78.7 \mathrm{~kg})$. Birds were housed in a large outdoor paddock $\left(5000 \mathrm{~m}^{2}\right)$ and provided with unlimited access to food and water. All experiments were performed in accordance with the Animal Ethics Committee of the University of Western Australia. Architectural and geometric data for the muscle-tendon units, and skeletal dimensions used in the computer model, were measured in a third female ostrich $(65.3 \mathrm{~kg})$, which had no musculoskeletal pathologies and was culled from a commercial ostrich herd (The Ostrich Meat Company, Merced, California). We subsequently verified these anatomical data by qualitative comparisons with three other adult specimens of qualitatively similar size, as well as literature descriptions (Gangl et al., 2004; Zinoviev, 2006).

\section{Kinematic measurements}


The experiments and 3D kinematic analyses are described in detail elsewhere (Rubenson et al., 2004,2007,2011). Briefly, the birds were trained to walk and run across a $50 \mathrm{~m}$ long fenced runway surfaced with high-density rubber matting $(10 \mathrm{~mm}$ thickness $)$. An eleven-parameter direct linear transformation (DLT) was used to construct a 3D image volume from two highspeed cameras $(200 \mathrm{~Hz})$ positioned at $45^{\circ}$ angles to the runway (Peak Motus; Peak Performance, Centennial, CO). The DLT was calculated using a custom-built 48-point moveable calibration frame. The video footage from the two cameras was gen-locked and synchronized manually using a hand held switch that caused a barcode in each video field to turn white. All data collection was performed after sunset under artificial lighting. A wide range of speeds was recorded in prior studies (Rubenson et al., 2004,2007,2011), but we only used representative (i.e., 1 trial/gait) kinematic data for a single walking trial at $1.22 \mathrm{~ms}^{-1}$ and slow running (at 3.46 $\mathrm{ms}^{-1}$ ). These two trials were within 1 S.D. of the mean kinematic data for running trials from Rubenson et al. (2007) and previously unpublished walking data from the same experimental analysis (see Results).

The 3D position and orientation of the ostrich limb segments were determined by videotaping clusters of non-linear, retro-reflective markers placed on the pelvis, femur, tibiotarsus, and tarsometatarsus and a single marker placed on the end of the third phalanx. These markers were used to define segment rigid-body Technical Coordinate Systems (TCS; Cappozzo et al., 1995). Prior to walking and running trials, static calibration trials were performed in order to identify several key anatomical landmarks necessary to construct segment Anatomical Coordinate Systems (ACS; see Rubenson et al. (2007,2011) for details). Anatomical landmarks were identified using a 6-marker pointer device and expressed in the segment TCSs. The static calibration trials thus allowed for the reconstruction of each segment ACS across walking/running strides using the motion data of the segment marker clusters alone.

Three-dimensional marker trajectory data were filtered using a fourth order zero-lag Butterworth low-pass filter (4-12 Hz) and compiled in c3d format (Motion Lab Systems, USA). Kinematics were computed using BodyBuilder modelling software (Oxford Metrics; Oxford, UK). Joint angles were calculated by determining the Euler angles associated with the transformation between the ACSs of the proximal and distal segments of a joint (Grood and Suntay, 1983). Segment ACSs in both the model (below) and in the experimental animals were constructed using the same landmarks (anatomical landmarks and numerically derived axes) and same ordered set of rotations between proximal and distal ACSs. Thus, 3D joint motion was defined equivalently in the musculoskeletal model and in the experimental animals and we were able to use experimentally derived joint angles as input into our musculoskeletal model to estimate muscular mechanics in vivo during walking and running.

\section{$3 D$ coordinate systems for anatomical dissection}

We used a Polaris optical tracking system (Northern Digital Inc., Waterloo, Ontario) to record the positions of anatomic landmarks and relevant joint kinematics during dissection sessions. This tracking system is accurate to within $1.5 \mathrm{~mm}$ with the $1.5 \mathrm{~m}^{3}$ measurement volume used in this study (Traxtal Inc., Toronto, Ontario), and in order to ensure this accuracy we performed appropriate calibrations before collecting our data.

We first skinned the right pelvic limb of the ostrich specimen. Before dissection of the muscles, we attached LED-emitting reference frames (AdapTrax trackers, Traxtal Inc., Toronto, Ontario) to each bone segment using orthopaedic bone screws. Each reference frame contained a cluster of LEDs that allowed the tracking system to record the 3D position and orientation of 
214 each segment (establishing the segment TCSs for the dissections, comparable to that for the 215 experiments). Figure 1 shows the apparatus we used. We used a digitizing probe (Northern 216 Digital Inc., Waterloo, Ontario) to digitize the 3D coordinates of the musculoskeletal geometry

217 in each session relative to these trackers. Unlike the LED-emitting reference frames, the

218 digitizing probe had a cluster of highly reflective spheres, making it an untethered and mobile tool. When these spheres were visible to the tracking system, the 3D position of the tip of the probe (calibrated in advance) could be recorded with respect to the TCS. Three rigid permanent points (marked with a drill as points on the bones) were measured on each segment to provide a local bone coordinate system for all digitizing/dissection sessions. This step allowed the TCS to be removed from the bone and reattached in a different area to facilitate the dissection process while still preserving the overall relationship of digitized points on a given bone between sessions.

Building a musculoskeletal model required points to be expressed in the segment ACSs (Figure 2 and Rubenson et al., 2007, 2011). The pelvis reference frame was defined as follows: the origin at the midline of the pelvis halfway between the left and right side hip joint centres; the unit vector SUL SYN (x-axis; positive being cranial); the cross-product of the unit vector SUL IL and the $\mathrm{x}$-axis (y-axis; positive being dorsal), and cross-product of the $\mathrm{x}$-axis and $\mathrm{y}$-axis (z-axis; positive being to the right). To locate the hip joint centres, we digitized 10-20 points in and around the acetabulum and femoral head, and then used least-squares optimization to fit a sphere to each of the two resulting point clouds. The centre of this best-fit sphere was the hip joint centre. To establish the reference frames for the other segments, we first estimated the medial-lateral joint rotational axis for the remaining joints by flexing and extending each joint and recording the $3 \mathrm{D}$ position and orientation of the distal bone with respect to the proximal one as a series of homogeneous transformation matrices. With these transformation matrices, we were able to calculate the average kinematic screw (helical) axes (Bottema and Roth, 1990) that best approximated the flexion-extension axis between those segments.

The femur coordinate system was defined as: the origin at the proximal joint centre; the segment $z$-axis along the medial-lateral joint rotational axis (positive being lateral); the $y$-axis as the cross-product of the $z$-axis and the unit vector between the proximal and distal joint centres; and the $\mathrm{x}$-axis as the cross-product of the $\mathrm{y}$ - and $\mathrm{x}$-axes. The tibiotarsus and tarsometatarsus coordinate systems were defined as: the origin at the proximal joint centre; the $y$-axis as unit vector between the proximal and distal joint centres; the segment $\mathrm{z}$-axis as the cross product of the medial-lateral joint rotational axis and the $y$-axis; and the $\mathrm{x}$-axis as the cross-product of the $\mathrm{y}$ and z-axes. The pes coordinate system was defined as: the origin at the proximal joint centre; the segment $\mathrm{x}$-axis as the unit vector between the proximal joint centre and the end of the segment; the $\mathrm{z}$-axis as the cross product of the medial-lateral joint rotational axis and the $\mathrm{x}$-axis; and the $\mathrm{y}$ axis as the cross-product of the $\mathrm{x}$ - and $\mathrm{z}$-axes. Putting any digitized points into these ACSs required two linear transformations: from the TCS into the local bone coordinate system and subsequently into the ACS. Table 1 provides data on axis positions used in the final model.

\section{Anatomical digitization and musculoskeletal model construction}

We began by dissecting the specimen proceeding from superficial to deep structures. The positions of muscles and bone geometry or other relevant anatomical features on each limb segment were measured using the digitizing probe. In particular, before removing muscles we digitized the circumferences of muscle origins and insertions as well as the 3D paths of the muscles from origin to insertion, using from 1-30 (depending on extent of the structure) $\mathrm{x}, \mathrm{y}, \mathrm{z}$ 
260

coordinate points to characterize each structure of interest. In addition, we measured other musculoskeletal features used as references, such as bone surfaces (for later alignment of complete 3D bone images from CT data), condylar contours, and those ligaments that influence muscle-tendon unit paths. All 3D points for use in the model were converted into the ACS.

After dissection the bones were de-fleshed and macerated, but with articular cartilages and menisci remaining as intact as possible. The bones were then CT scanned (1.5-3 $\mathrm{mm}$ slices, 120-130 kPa, 109-150 mA, on a Picker PQ5000 CT scanner) and the resulting slices digitized in Mimics software (Materialise, Inc; Leuven, Belgium) to produce rendered 3D polygonal meshes. Ultimately ASCII (ASC) format images were used (decimated to $<50,000$ polygons each) as bone images in the model. Importantly, the bones were only visual aids and not inherent obstacles to joint or muscle motion, but they are crucial for visualizing musculoskeletal function.

We used these anatomical data and bone images to construct a 3D musculoskeletal computer model of the right pelvic limb using SIMM software (Musculographics, Inc.; Chicago, IL; Delp et al., 1990, 1992; Delp and Loan, 1995, 2000). The right limb was mirrored as a left limb. The digitized muscle paths were used as a template for developing the muscle paths in the final model (Figures 3, 4). Paths were represented using a combination of "via points" (i.e., static points fixed relative to a segment) and "wrapping surfaces" that prevented translation of points outside of a predefined area (see Delp and Loan, 1995, 2000 for details; also Hutchinson et al., 2005, for a similar procedure we used for Tyrannosaurus rex). Table 2 presents the muscles modelled, with abbreviations used throughout the paper. Table 3 describes all the muscle wrapping surfaces assumed in the model and Figure 5 displays examples.

Inevitably, because the 3D musculoskeletal geometry was complex, we had to cautiously judge where to position wrapping surfaces and what size and shape they should be. Additional ostrich cadaveric material was used along with the literature (Gangl et al., 2004; Zinoviev, 2006) to qualitatively refine the model as we iteratively progressed, checking that paths and attachments were represented reasonably and consistently. We took care to visualize the model in many different 3D joint positions to ensure that muscles did not pass through areas occupied by other soft tissues or especially bones and to eliminate other numerical errors generated by interactions of the muscle-tendon unit paths with wrapping surfaces (e.g., "loops" in muscles caused by contradictory constraints in the model). Importantly, because we intended to compare our model's results with data from Smith et al. (2007) and Bates and Schachner (2012), we kept our model construction blind to the results of these studies, avoiding any comparisons and indeed finishing the major steps in completing our model before these studies were published.

\section{Muscle-tendon unit architecture and physiology}

After we dissected, digitized, and removed the muscles, we separated them from their proximal/distal tendons and other connective tissue. We then used digital calipers $( \pm 0.1 \mathrm{~mm})$, an electronic balance $( \pm 0.001 \mathrm{~g})$, and a protractor $\left( \pm 1^{\circ}\right)$ to measure muscle fascicle lengths $(L)$, masses $\left(m_{\text {musc }}\right)$, and resting pennation angles $(\theta)$ for calculating physiological cross-sectional area $\left(A_{\text {phys }}\right)$, taking an average of five randomized measurements for $L$ and $\theta$ in larger muscles.

Using water displacement (immersing sectioned muscles in graduated cylinders) to calculate muscle belly (sans tendon) density (d) from (volume $m_{m u s c}{ }^{-1}$ ), we obtained a mean value of $1.0645 \times 10^{3}(\mathrm{n}=10 ; \mathrm{S} . \mathrm{D} .=0.0347) \mathrm{kg} \mathrm{m}^{-3}$, matching measurements of mammalian muscle (Mendez and Keys, 1960; Brown et al., 2003a). Hence we assumed a conventional value of $d$ as $1.06 \times 10^{3} \mathrm{~kg} \mathrm{~m}^{-3}$. As commonly practiced, we assumed $L$ to be equivalent to optimal fiber length 
305

306

307

308

309

310

311

312

313

314

315

316

317

318

319

320

321

322

323

324

325

326

327

328

329

330

331

332

333

334

335

336

337

338

339

340

341

342

343

344

345

346

347

348

349

350

( $l_{o}{ }^{m} ;$ Zajac, 1989). We could thus calculate $A_{\text {phys }}$ as (e.g., Alexander et al., 1979; Lieber \& Boakes, 1988a; Brown et al., 2003b; Hutchinson, 2004):

$$
A_{\text {phys }}=m_{\text {musc }} \cos \theta(L d)^{-1} \quad \text { (Equation 1) }
$$

Equation 2 then estimates $F_{\max }$, maximum isometric force capacity:

$$
F_{\max }=3.0 \times 10^{5} \mathrm{~m}^{-2} A_{\text {phys }} \quad \text { (Equation 2) }
$$

In which the constant is isometric stress under maximal activation (Medler, 2002; Nelson et al., 2004). Note that the musculoskeletal model varies $\theta$ with $L$ to maintain constant muscle thickness (Zajac, 1989). Muscle maximal contraction velocity was not calculated in the present model, but could be added (see Smith et al., 2006 for example) for more complex simulations, using published fibre type data for ostriches (Velotto and Crasto, 2004) and ostrich or other avian muscle force-velocity data (e.g., Nelson et al., 2004). We also did not include measurements of tendon force-length data here, but either dimensionless estimates (Zajac, 1989) or specific measurements can be added to future simulations. For simplicity, we chose to focus in this initial study on near-isometric muscle action and progress to more complex, dynamic parameters in later work.

Muscles were identified as in Table 2 following Gangl et al. (2004) and Zinoviev (2006) (see Appendix 1 for additional details). For simplicity, we combined some muscle heads into single functional muscles in the model (cf. Gangl et al., 2004:table 1) and omitted some tendinous/minute muscles which were grouped with other similar muscles nearby or omitted in the case of M. popliteus (rotates fibula around tibia; Fuss, 1996); these simplifications are outlined in the Supplementary Text. However, some muscles (e.g., M. iliofibularis, M. iliotrochantericus caudalis) were large enough that separation into two heads was deemed important, as some heads might have very different flexor/extensor moment arms than more cranial/caudally-positioned ones.

\section{Limb muscle biomechanics: Calculations and hypothesis testing}

The musculoskeletal model was then imported into OpenSim (opensim.stanford.edu) software in order to take advantage of the programme's established analysis capabilities. OpenSim uses the 'virtual work' method (change of muscle-tendon unit length per unit joint rotation) explained by Delp and Loan $(1995,2000)$ and Pandy (1999) to compute muscular moment arms over a range of motion. Maximal muscular moments then can be estimated using muscle $F_{\max }$ and potentially $l_{o}^{m}$ (see above and Zajac, 1989).

To test whether ostrich muscle moment-generating capacity is optimized to match peak loads during walking and running (our Question 1), we compared the results from estimated maximal muscle moments to experimentally-calculated internal and external moments (Rubenson et al., 2011), addressed in the Discussion. First, each muscle's maximal isometric muscle force $\left(F_{\max }\right)$ was multiplied by the flexor/extensor moment arm calculated by OpenSim (i.e., from the individual trials' limb joint angle input data and the model's resulting moment arm output data), for each pose adopted throughout the representative walking and running gait cycle trials (every $1 \%$ of gait cycle) to obtain the relationship between locomotor kinematics and isometric muscle moments. Second, OpenSim was used to calculate individual muscle moments directly, taking into account muscle force-length relationships (set as dimensionless in a Hill 
377

378

379

380

381

382

383

384

385

386

387

388

389

390

391

392

393

394

395

396

model as per Zajac, 1989), in order to provide a more realistic estimate of the variation of maximal moment-generating capacity throughout the same gait cycles. Both approaches were static, ignoring time/history-dependent influences on muscles. The second approach allowed non-isometric muscle action to be represented, but did not incorporate force-velocity effects, which would require a more dynamic simulation to resolve. Total extensor and flexor maximal moments were calculated in OpenSim as well as the net (extensor + flexor) maximal moment.

To determine if ostrich limb muscle moment arms peak at extended limb orientations or at mid-stance of locomotion (our Question 2), we used the model to calculate the mean moment arm of all extensor or flexor muscles across the full range of motion of each joint (estimated from osteological joint congruency as in Bates and Schachner (2012)) in flexion/extension (set at constant values for mid-stance of running in other degrees of freedom), summed these mean moment arms, and divided that sum by the summed maximal moment arms for each muscle across the same range of motion (as in Hutchinson et al., 2005). We then inspected whether our representative mid-stance poses in walking or running matched maximal or minimal averaged moment arms corresponding to those poses.

To compare the degree of matching between muscle moment arms in our model and the experimental data of Smith et al. (2007) and Bates and Schachner (2012) (our Question 3), we obtained the published experimental and modelling data (K.T. Bates, provided by request), transformed their joint angle definitions to be consistent with our model definitions, and plotted the muscle moment arms vs. each joint angle with our moment arm data (also see Supplementary Figures S1-S4), restricting the other studies' ranges of motion to those presented in the original studies. For the knee and joints distal to it, in this study we focus only on flexor/extensor moment arms for simplicity and because the importance of long-axis and ab/adduction muscle (vs. passive tissue) moments at these distal joints is unclear, although our model could be adjusted to calculate those non-sagittal moment arms and moments.

\section{Results}

Here we present our data for addressing our main questions, proceeding in order with maximal muscular moments, maximal/minimal moment arms, and then general moment arm patterns compared with other studies'. The model is downloadable from the repositories at https://simtk.org/home/opensim and Figshare (Hutchinson et al., 2015) and can be manipulated in open source software OpenSim. Supplementary Movie S1 shows the model animated through the representative running stride (from Rubenson et al. (2007)). Supplementary Figure S5 shows the kinematic data for the walking trial vs. a larger dataset (not shown in Rubenson et al. (2007), but obtained from the same methods and experiment). In that representative trial, the pelvic pitch angle is $\sim 10^{\circ}$ more tilted (pitched upward) than the mean-- reasons for this isolated deviation are unclear but also very unlikely to influence our findings here. Both individual walking and running trials' data are supplied in the repository (Hutchinson et al., 2015).

\section{Maximal muscular moments}

Our 3D ostrich limb model predicted how the maximal capacity to generate muscle moments should vary with limb orientation during walking and running (Figures 6,7). Maximal flexor moments increase if force-length properties are ignored (treating all muscles as isometric). This indicates that most muscles in the model are at disadvantageously short fibre lengths during locomotion, with walking having a generally greater capacity for flexor moment generation 
397 (especially about the hip) than running. These curves do not change much across the gait cycle.

398 The pattern for extensor moments is more complex. Peak capacity tends to be in late swing phase 399 (reasonably consistent across all joints). Force-length properties here provide an advantage, 400 presumably because the muscles are lengthened. Data during the stance phase do not support the 401 hypothesis, regardless of assumptions about muscle-force length states, that postures used around mid-stance of walking or running optimize the moment-generating capacity of pelvic limb muscles in ostriches: the maximal moments early or late in stance phase, and late in swing phase, are of similar or greater magnitudes. The relatively flattened shapes of most moment curves without force-length properties enforced ("Fmax"; dotted lines in Figures 6,7) indicate that muscle moment arm variation across postures used in vivo during locomotion is a smaller contributor to moment generation than force-length properties ("F-L"; solid lines) in Struthio.

\section{Maximal/minimal muscle moment arms and limb orientation}

Do ostriches' limb muscle moment arms peak at very extended limb orientations or at mid-stance of walking/running (Figure 8)? We find that the mean hip extensor moment arms decrease from a peak at full extension as hip joint flexion increases, and the hip flexors behave similarly. However, knee and ankle moment arms each exhibit different patterns. The knee extensor and flexor moment arms tend to peak at moderate knee flexion angles $\left(\sim 60-90^{\circ}\right)$, as do the ankle extensors (plantarflexors), but the ankle flexors have a near-plateau for most angles, quickly decreasing with extreme dorsiflexion $\left(>100^{\circ}\right.$ ankle angle).

When the poses that ostriches use during periods of peak limb loading (near mid-stance of walking and running; Rubenson et al., 2007) are compared against these patterns (Figure 8), it becomes evident that there is no clear optimization of muscle moment arms for supportive (large extensor or small flexor values) roles during these periods of potential biomechanical constraints. This is in agreement with the maximal moment data from Figures 6 and 7. Hip extensors and flexors as well as ankle extensors are relatively far ( $\sim 60-85 \%$ of maximal mean moment arms) from optimal values at mid-stance of walking and running. Knee extensor/flexor moment arms are closer to maximal values, especially for walking. However, the co-contraction of multiarticular hip extensor/knee flexors (e.g. ILFB, FCLP) against knee extensors would eliminate associated benefits-i.e., the ratio of peak knee extensor to peak knee flexor moment arms would have not have minimized the net knee extensor moments required at mid-stance of either walking or running. At moderate knee flexion values, both the capacity of muscles to extend and to flex the knee are near-maximal (Figure 8).

\section{Moment arms: general trends and comparisons with prior studies}

Figures 9-11 show our results for hip flexion/extension moment arms of ostrich muscles, with comparable data from Smith et al. (2007) and Bates and Schachner (2012) also plotted if available (abbreviated in this section as S.E.A. and B.A.S. respectively). Here we focus on the major findings. The two AMB muscles (Figure 9) compare reasonably well among all three studies, showing a decrease of hip flexion moment arms at strongly flexed limb poses and in some cases (our AMB1,2 and the AMB of B.A.S.) a switch from flexor to extensor action with flexion $\left(\sim 30-90^{\circ}\right)$. The IC muscles likewise have reasonably comparable results, but only our IC muscle switches action at extreme flexion. Our model agrees well with the data of S.E.A. and especially B.A.S. for the IL muscle, including its decreasing hip extensor moment arm with increasing hip flexion and a switch from hip extensor to flexor action at typical in vivo positions $\left(\sim 40-70^{\circ}\right)$. We have similar findings for the ILFB muscle, although no switch to hip flexor 
443 moment arms is observed in either of the two parts of this muscle in our model (S.E.A. and

444 B.A.S. represented it as one part) (Figure 9). IFE, IFI, ISF and OM muscles (Figure 10). We find fair agreement among studies for the IFE (note confusion caused by misidentification of muscles in prior studies-- see Appendix 1; the "IFE - Smith" in Fig. 10 is equivalent to our IFE and ITC), ITC, IFI, ITM and ITCR muscles' general changes of moment arms. Our IFE moment arm values are smaller than for S.E.A. and B.A.S. apparently because of the aforementioned identification issue (the top left panel in Fig. 10 shows our IFE plotted against S.E.A.'s IFE+ITC combined). Notably, the curves for the two parts of ITC in our data and those of B.A.S. are remarkably similar (and consistent with S.E.A.'s experimental data for their "IFE - Smith" as well as "ITC - Smith") despite the subjectivity inherent in partitioning this large muscle into two paths. These moment arms grade from flexor to extensor action with strong flexion $\left(\sim 40-70^{\circ}\right)$. A similar trend is evident for the ITM and ITCR muscles (but note the identification issues outlined in Appendix 1; S.E.A.'s "ITC" is actually the ITM, which their data otherwise lacks, so the top right panel in Fig 10 compares their actual ITM ["ITC - Smith"] vs. our ITM). The antagonistic OM and ISF muscles concur less closely between the latter two studies, however, displaying more convex curves tending to indicate hip flexor action in our data, with more concave, flattened arcs favouring hip extensor action in B.A.S. (Figure 10).

The "hamstring", caudofemoral and adductor hip muscles uniformly display extensor action, befitting their more caudal paths relative to the hip, but agree less well among studies than the prior muscles (Figure 11). Our data for the FCM, FCLP, CFP and PIFML muscles portray peak moment arms at low hip extension angles $\left(\sim 0-30^{\circ}\right)$, decreasing with flexion away from these ranges. These trends qualitatively agree with the S.E.A. and B.A.S. data, but moment arm values tend to be substantially smaller in those data, especially for the FCLP and FCM muscles. Our PIFML data show less variation with joint angle than the S.E.A. and B.A.S. data because we had to constrain this muscle's path in 3D to avoid it cutting through bones or other obstacles in some poses. Note also how the S.E.A. results in general show strong changes with joint angles, whereas the more constrained muscle geometry of our model and B.A.S.'s results in more modest changes (Figure 11).

Long-axis rotation (LAR; in Figures 12,13) moment arms for hip muscles only allow comparisons between our data and those of B.A.S . Furthermore, considering that B.A.S. plotted these moment arms against hip flexion/extension joint angle (modified data shown; Karl T. Bates, pers. comm., 2015), we show them that way here but also plot them against hip LAR joint angle in the Supporting Information (Supplementary Figures S1,S2); however we do not discuss the latter results here. For the AMB1,2 muscles we find consistently weak, near-zero LAR action (lateral/external rotation), whereas B.A.S. showed a steeply decreasing hip medial/internal LAR moment arm as the hip is flexed (Figure 12). In contrast, our IC and IL muscle data agree well with B.A.S.'s in having a shallow increase of the medial/internal LAR moment arm with hip flexion, although B.A.S.'s data much more strongly favour a medial rotator function for the IC muscle. Our results for the two parts of the ILFB muscle are very different from B.A.S.'s in trending toward stronger medial/internal rotation function as the hip is flexed, whereas B.A.S.'s favour lateral/external rotation. The results for the OM muscle have better matching between studies, indicating a lateral/external rotation action for this large muscle. Likewise, our ISF data and those of B.A.S. match fairly closely, with consistent lateral/external rotator action. The FCM and FCLP muscles have among the largest LAR moment arms for all muscles $(\sim 0.08 \mathrm{~m}$; also 
489

490

491

492

493

494

495

496

497

498

499

500

501

502

503

504

505

506

507

508

509

510

511

512

513

514

515

516

517

518

519

520

521

522

523

524

525

526

527

528

529

530

531

532

533

534

observed for our ILp muscle) in our data, but both muscles reduce their lateral rotator action with increasing hip flexion. In B.A.S.'s data a weaker, opposite (medial/internal rotator) trend with hip flexion was found for the FCM, whereas the FCL muscle maintained a small lateral/external rotator action (Figure 12).

The uniarticular hip muscles' LAR moment arms of our model tend to switch less often (at in vivo hip joint angles $\sim 30-60^{\circ}$; e.g. Supplementary Figure S5) from medial to lateral rotation or vice versa (Figure 13). The IFI, however, remains mainly as a weak medial rotator except at extreme hip flexion $\left(>60^{\circ}\right)$. B.A.S.'s data favoured stronger medial/internal rotation moment arms for the IFI but otherwise had a similar pattern. Our IFE muscle's data indicate a switch from lateral rotation into medial rotation near a $30^{\circ}$ hip flexion angle, matched fairly closely by B.A.S.'s data. Our results for the two-part ITC muscle concur qualitatively with B.A.S.', consistently having a strong medial/internal rotator action but smaller at more extended joint angles. As in B.A.S.'s data, but featuring smaller moment arms, our data show that the CFP and PIFML muscles have consistent lateral/external rotation action in ostriches; decreasing with increased hip flexion. The ITM and ITCR's medial rotator moment arms peak at hip angles of 30-60, then decrease; a pattern qualitatively matched by B.A.S.'s data. (Figure 13).

Abduction and abduction moment arms for the hip muscles show strong postural dependency like the LAR moment arms do (Figures 14-15). Again, as for the LAR data above, we supply these data plotted against abduction/adduction hip joint angle in the Supporting Information (Supplementary Figures S3, S4), but we do not discuss those results here. The PIFML muscle has a discontinuity in its hip abductor moment arm (Fig. S4) in our model at extreme hip abduction angles $\left(>-40^{\circ}\right)$ but this is well outside normal in vivo abduction angles used $\left(<25^{\circ}\right.$; Rubenson et al., 2007). The two AMB muscles in our model have peak adductor moment arms at different flexion angles $\left(\sim 30^{\circ}\right.$ and $\left.80^{\circ}\right)$, then decrease. B.A.S.'s data (modified data shown; Karl T. Bates, pers. comm., 2015) generally follow our AMB1 muscle's. Our IC muscle has a similar adductor moment arm curve as our AMB2, and a similar divergence from B.A.S.'s results, which remain close to a zero moment arm. Our IL muscle parts (ILa,p) agree well with B.A.S.'s, showing them to act as abductors. Both our ILFB muscle parts (ILFBa,p) have little variation in their hip abductor actions, whereas B.A.S.'s representation had a $\sim 100 \%$ larger moment arm but otherwise was similar. The OM muscle, which runs very close to the plane of the acetabulum, is an adductor at extended joint angles and an abductor at flexed angles in both our model and in B.A.S.'s data. Whilst the ISF muscle is almost exclusively a hip abductor in our model, it was exclusively an adductor in the B.A.S. model. The FCL and FCM muscles compare only qualitatively between our data and B.A.S.'s, remaining as hip abductors. It is noteworthy that throughout the full ranges of hip motion we examined, most muscles would act as hip abductors; the dorsal AMB2 and IC muscles are the only consistently strong hip adductors (Figure 14; Supplementary Figures S3, S4).

Uniarticular "deep dorsal" and antagonistic muscles show similar trends as the above muscles for adduction/abduction capacities (Figure 15). The IFI has weak adductor action, vs. a smaller, near-zero value (but similar trend) in B.A.S.'s data, whereas our data and B.A.S.'s agree well on the hip abductor moment arm of the IFE. Our representations of the ITCa/p muscle parts switch from abduction to adduction function as hip flexion surpasses $45-60^{\circ}$; B.A.S.'s model did this switch to a stronger degree. Postacetabular muscles such as the CFP and PIFML in our model are almost exclusively hip abductors, much as in B.AS.'s model. Finally, our results also generally have a good match to B.A.S.'s in the case of the ITM and ITCR muscles, which convert from abductor to adductor action at $10-40^{\circ}$ hip angles (Figure 15). 
535

536

537

538

539

540

541

542

543

544

545

546

547

548

549

550

551

552

553

554

555

556

557

558

559

560

561

562

563

564

565

566

567

568

569

570

571

572

573

574

575

576

577

578

579

580

We only focused on flexion/extension moment arms for more distal joints, starting with the knee (Figures 16,17). Good agreement between knee extensor moment arms for the AMB2 (dorsal) muscle is evident with the two other studies. The AMB1 (ventral) component only has data from our model (mainly a weak knee flexor), as does the IC (very weak knee flexor/extensor at flexed/extended angles). We estimate a larger knee extensor moment arm for the FMTL muscle but this is because of misidentification of part of that muscle in the S.E.A. and B.A.S. data (only a distal head was included in this muscle; see Appendix 1). Similar differences of anatomical representation are likely explanations for the deviation between our result (weak knee flexor) and S.E.A.'s (strong knee extensor) for the FMTM (see Discussion). Our model presents slightly different moment arms for its two IL muscle heads, peaking in extensor values at 30-90 flexion, whereas B.A.S. had identical moment arms increasing throughout extension.

We estimate the knee flexor moment arms as identical (peaking at $90-120^{\circ}$ flexion) for the two parts of the ILFB muscle in our model, which match S.E.A.'s data well, whereas B.A.S. had moment arms switching from extensor to flexor at $70^{\circ}$ of knee flexion, peaking at quite extended knee poses (Figure 16) (see Discussion). Other "hamstring" muscles (no comparable data for S.E.A. or B.A.S.) include the FCM and FCLP, which shift steeply from knee extensor to flexor moment arms at high flexion angles and then peak near $90^{\circ}$ in its flexor moment arm value (Figure 16). The FL muscle shows an almost mirror image pattern, acting as a knee extensor. Muscles running past the ankle joint (Figure 17), such as the FPD3 and FPD4 groups, have a similar pattern to the FCM and FCLP at the knee, but the TCf muscle has almost no knee moment arm; consistently acting as a very weak extensor. Finally, parts of the gastrocnemius muscle group (e.g. GIM) reach peak knee flexor moment arms of about $0.07 \mathrm{~m}$ at intermediate knee flexion angles $\left(60-90^{\circ}\right)$. The data for S.E.A. and B.A.S. and for our GL muscle remain(ed) near smaller knee flexor values, with less postural variation (Figure 17).

Ankle musculature displays fairly congruent patterns in our model and S.E.A. and B.A.S.'s data (Figures 18,19). The TCf and TCt heads generally have an ankle extensor action, like the EDL muscle group does, albeit with some switches to extensor action with extreme (dorsi)flexion in the B.A.S. dataset (and our TCf). Surprisingly, ankle extensors reveal more variation: our FDL's ankle extensor moment arm is almost twice as large of that in the S.E.A. and B.A.S. data, showing little change with ankle posture, whereas the B.A.S. dataset exhibited a decreased moment arm with flexion. Our other digital flexor muscles (FPD3, FPD4) and those of S.E.A. display roughly similar values but opposite trends, increasing their moment arms with ankle flexion in our model. Our FL muscle's extensor moment arm is smaller than those of S.E.A. and B.A.S. The model of B.A.S. had a M. fibularis brevis (FB) muscle (Figure 18), which is reduced to a ligament in Struthio and thus not included in our model; no studies have data for the ligamentous M. plantaris (Zinoviev, 2006). The extensor moment arms for our gastrocnemius muscles are all identical and fairly constant with ankle flexion, whereas the curves for the data of S.E.A. and B.A.S. increased steadily and tended to be larger (Figure 19).

Digital flexor muscle moment arms all stay fairly constant (slight increase with extension of the MTP joint) in our model whereas they showed a stronger decrease in S.E.A.'s experiment (Figure 20). Our EDL muscle has stronger moment arms than in S.E.A.'s data but a similarly shallow curve. Finally, our FL muscle exhibits digital flexor moment arms similar to those of the other digital flexors.

\section{Discussion}


581

582

583

584

585

586

587

588

589

590

591

592

593

594

595

596

597

598

599

600

601

602

603

604

605

606

607

608

609

610

611

612

613

614

615

616

617

618

619

620

621

622

623

624

625

626

The results of our combined experimental and theoretical approach show first that, while ostrich limb muscles are capable of generating large flexor and extensor moments about their limb joints during locomotion (Figures 6,7), they do not seem to match maximal muscle moment-generating capacity with instants of peak loading in walking or slow running. Second, the moment arms of ostrich flexor/extensor muscles often change greatly with limb orientation, but they are not consistently matched to minimize the former and maximize the latter during key periods of weight support in locomotion (Figure 8). Third, there is mostly reasonable consistency in three different studies of ostrich muscle moment arms (Figures 9-20), indicating at least fair repeatability with distinct methods, but still some striking disagreements, especially in the littleexplored area of non-flexor/extensor muscle mechanics. We explore these topics in more detail below and then consider broader issues related to our findings.

\section{Maximal muscle moments and kinematics}

Our Question 1 asked, "Do ostriches adopt limb orientations during walking or running that optimize their capacity to generate maximal moments about the pelvic limb joints?" We find no convincing evidence of such optimization -- maximal capacities to produce joint moments often peak either early in stance phase or during swing phase (Figures 6,7). In both cases, net joint moments obtained from inverse dynamics analysis are low (Rubenson et al., 2011). Peak flexor moments (requiring extensor/antigravity muscle activity) occur at or near mid-stance in running ostriches (Rubenson et al., 2011:figure 7), reaching magnitudes $>-2 \mathrm{Nm} \mathrm{kg}^{-1}$ (i.e. $>-150$ $\mathrm{Nm}$ for our subject). Note that these magnitudes are far below the capacities of hip, knee and ankle muscles $(>400 \mathrm{Nm}$; Figures 6,7$)$ but approach those of the digital flexors $(\sim 150-200 \mathrm{Nm}$; Figure 7). On this basis, we infer that either passive tissues (including muscle passive forcelength properties) play an important role in balancing moments about the tarsometatarsophalangeal joint in running ostriches (cf. Haughton, 1864; Schaller et al., 2009,2011 ), especially at faster speeds, or that muscle moment-generating capacity is near its limits for this joint in particular, even at slower speeds. Nevertheless, more proximal limb muscles seem further from their moment-generating limits.

In his classic biomechanical analysis of ostrich anatomy, Haughton (1864) assumed that "the greatest possible amount of muscular force shall be expended in straightening or unbending the legs", and thus that early and late stance respectively placed the greatest demands on these forces. Available data no longer support this notion, but there is no question that ostriches have muscle masses able to produce greater moments (and work) in extension than in flexion, as Haughton explained, but by a factor of about three times for the hip and knee rather than ten (vide Smith et al., 2006,2007).

There are several potential explanations for our observations that lead us to a negative answer to our study's first question. First, we have only examined walking and slow running. Near maximal speed, moment capacity and requirements around mid-stance might be more closely matched (e.g. Hutchinson, 2004), as forces surely increase. At a duty factor of 0.42 , Rubenson et al. (2011) obtained peak vertical ground reaction forces of 1500-2000 N or about 2.17-2.89 times body weight (BW), whereas Alexander et al. (1979) estimated 2.7 BW peak forces for an ostrich at near top speed (duty factor 0.29 ). The latter study used an equation that probably underestimates peak forces for ostriches, as Rubenson et al.'s (2011) data show (peak forces are 16-55\% greater than predicted from duty factor). Second, our present model is still static, not considering force-velocity or other dynamic interactions that would alter moment- 
627 generating capacities. It is possible that these parameters, or highly complex interactions (e.g., 628 muscle moment arms and "power amplification"), could be more influential than the isometric and force-length properties that our model considers. Third, entirely different factors could determine locomotor and postural optimization, such as energetic costs or stability/manoeuvrability (e.g., Daley and Usherwood, 2010).

Comparison of our results with other studies of the relationship between limb orientation and muscle mechanics reveal a fourth potential explanation, that the optimization of anatomy, posture, physiology and other factors in locomotor dynamics could be highly species-, task-, limb-, joint- or muscle-specific. Lieber and colleagues (Lieber \& Boakes, 1988a,b; Mai \& Lieber, 1990; Lieber \& Brown, 1992; Lieber \& Shoemaker, 1992) conducted an elegant series of studies that constitute a model system for addressing this issue. They elucidated that maximal moment production by the semitendinosus muscle in frog hindlimbs showed a strong dependence on muscle isometric force capacity and moment arms. Some of these studies found less dependence of moment production on joint angle-dependent moment arm values (e.g., Lieber \& Boakes, 1988a,b), but this dependency varied for the hip and knee joints (Mai \& Lieber, 1990; Lieber and Shoemaker, 1992) - and might be expected to vary for other muscles, too. Indeed, the moment arm did not vary much with knee joint angle for the semitendinosus (e.g., $0.37-0.44 \mathrm{~cm}$ about knee, across $10-160^{\circ}$ range of flexion/extension; Lieber \& Boakes, 1988a:Figure6A) so this muscle could not contribute much variation to muscle moment production. One might predict more dependency of maximal muscle moment production on moment arms for muscles that have more variable moment arms, but this has not been conclusively determined. Lieber \& Brown (1992) found that there was no simple relationship between muscle fibre length and moment arm in seven frog hindlimb muscles, with differences evident between muscles acting about the hip and knee, suggesting diverse adaptations to moment production demands.

The aforementioned studies' reviews of numerous others in humans, cats and other species likewise note some variability and uncertainty in what factors determine maximal moment capacity in limbs, so consensus has been elusive. Furthermore, Brown et al.'s (2003b) modelling/experimental study of horse forelimbs found that while the moment-producing capacities of flexor muscles were determined mostly by muscle properties (e.g., $F_{\max }$ or muscle force-length), muscle moment arms could have greater effects on moments than those properties for some extensor muscles. Young et al. (1993) also found variability from $50-100 \%$ of resting fascicle length in the amount of length change that cat ankle muscles used throughout their range of motion. Thus strict "sarcomere equivalency" (constant usage of maximal muscle range of motion; e.g. discussions in McClearn, 1985; Lieber, 1997; Bates \& Schachner, 2012) is not expected, but an approximate "tuning" of moment arms to muscle fibre lengths (and joint ranges of motion) is expected at least in some cases (see Sellers et al. (2013) for an example of how such information could be useful). Lieber and Shoemaker (1992) explained how a greater muscle fibre length to moment arm ratio would cause muscle force-length properties to become less influential on muscle moment production. Therefore, in the long-fibred proximal muscles of ostriches and other birds that run closer to the hip and knee joints and thus have smaller moment arms (e.g. Smith et al., 2006, 2007), individual muscles' moment arms might be quite influential. This speculation has yet to be conclusively tested, let alone integrated into studies of whole limbs and locomotor dynamics.

Regardless, recent studies of the hindlimbs of mice (Lieber, 1997), rats (Johnson et al., 2008), and chimpanzees (O'Neill et al., 2013), as well as horse forelimbs (Brown et al., 2003a,b) favour some optimization of locomotor tasks and muscle moment arms, as do broad comparative 
673

674

675

676

677

678

679

680

681

682

683

684

685

686

687

688

689

690

691

692

693

694

695

696

697

698

699

700

701

702

703

704

705

706

707

708

709

710

711

712

713

714

715

716

717

718

studies of elbow muscles by Fujiwara and colleagues (Fujiwara, 2009; Fujiwara et al., 2011; Fujiwara and Hutchinson, 2012) and a comparative study of mammalian carnivores (McClearn, 1985). So far, however, general principles that extend across lineages, behaviours or anatomies remain elusive. We consider this ambiguity's effect on inferences about extinct taxa further below.

\section{Muscle moment arm-joint angle dependencies}

Next, we posed Question 2: Are the moment arms of limb muscles maximized (for antigravity/extensor muscles; or minimized in the case of antagonistic flexor muscles) at midstance of locomotion (optimizing weight support), or at highly extended limb orientations, as prior studies of dinosaurs inferred (e.g., Hutchinson et al., 2005)?

The peak extensor muscle moment arms that ostrich pelvic limb antigravity muscles have about the hip joint lie close to a completely columnar (i.e., vertical or $0^{\circ}$ ) hip angle, approximately $5^{\circ}$ (Figure 8), similar to prior results for Tyrannosaurus rex (Hutchinson et al., 2005). Ostriches, however, do not stand or normally move with such extended hip joints (Rubenson et al., 2007). We suspect this difference is because of their two orders of magnitude smaller body size (65+ $\mathrm{kg}$ vs. $\sim 6000+\mathrm{kg}$ ) and hence the lack of necessity for extreme postural changes to maintain lower muscle stresses in order to maintain locomotor performance (Biewener, 1989, 1990). However, ostriches may also have a greater importance for nonisometric muscle force-length properties in determining the limb orientation used (Figures 6,7), as per the section above. Such speculations can be tested better once such physiological data exist for ostrich muscles. Our data also do not strongly support Smith et al.'s (2007) suggestion that hip extensor (or other muscle) moment arms are at peak values toward the end of stance phase (Figures 6-8).

Overall, unfortunately the factors that determine limb orientation in locomoting ostriches, as the largest extant striding biped (and theropod dinosaur) available for study, remain inconclusive, leaving the application of such principles to reconstructing limb orientations and locomotion in extinct theropods (e.g., Hutchinson et al., 2005; Gatesy et al., 2009) on shakier empirical and theoretical ground. However, this uncertainty is not cause for cynicism. It is an opportunity for future improvement, especially given the dearth of comparative studies that focus on how musculoskeletal mechanics relate to limb orientation, and the technical difficulties inherent to measuring or modelling muscle moment arms and other properties. Furthermore, quantitative biomechanical studies of extant or fossil organisms should still be considered a major step forward from past qualitative, intuitive or subjective functional studies.

\section{How accurate and repeatable are estimates of ostrich limb muscle moment arms?}

Our study's Question 3 dealt with a methodological comparison among the three main studies of ostrich pelvic limb muscle moment arms. Agreement seems fair overall, especially for flexion/extension actions. However, several main messages emerge from our comparisons, some of which were also voiced by the other two studies of ostrich pelvic limb moment arms (Smith et al., 2007; Bates \& Schachner, 2012; here "S.E.A." and "B.A.S."). Circumstantial support for all three methods' accuracy additionally comes from tendon travel measurements of cranial and caudal parts of the IL muscle in guineafowl by Carr et al. (2011). General patterns (their Figure 7) for the IL moment arms about the knee (concave arc, peaking $\sim 100^{\circ}$ knee angle in flexion) and the hip (increasing with extension) agree reasonably well with these three ostrich studies (Figures 12, 16). However, all moment arms for the ostrich IL muscle infer a switch to hip flexor 
719

720

721

722

723

724

725

726

727

728

729

730

731

732

733

734

735

736

737

738

739

740

741

742

743

744

745

746

747

748

749

750

751

752

753

754

755

756

757

758

759

760

761

762

763

764

action in strongly flexed poses, and little or no levelling off of the moment arm curve at strong hip flexion angles.

Key areas of disagreement between our results and those of B.A.S. and/or S.E.A. include occasionally major differences in if, or how, muscles switch between flexion and extension (e.g., the AMB1 and AMB2, IC, ILFB about the hip; Figures 9-11), whether certain muscles are flexors or extensors (e.g., the OM; see "Implications for ostrich limb muscle function" below), or the absolute magnitudes or relative trends in the data (e.g., our near-constant moment arms about the ankle for the FDL and gastrocnemius muscles; Figures 18,19; and for the digital muscles, Figure 20). We also found some differences in LAR and ab/adduction moment arms about the hip for B.A.S.'s data, but these are likely explained by differing muscle paths (e.g. via points and wrapping); see Figures 12-15. Bates and Schachner (2012) acknowledge that their model could not use both via points and wrapping surfaces for the same muscle. This limitation explains the switch of their ILFB knee moment arm from flexor to extensor with knee flexion (unlike Smith et al.'s (2007) data); our model only exhibits this switch at extreme knee flexion $\left(\sim 150^{\circ}\right.$ vs. $90^{\circ}$; Figure 16). Similarly, our FDL's ankle extensor moment arm was nearly constant (Fig. 18), as in Smith's data, but the modelling limitation might explain why Bates and Schachner's (2012) moment arm curve showed a stronger decrease with ankle flexion.

Contrastingly, the "M. femorotibialis medialis" (see Appendix 1; equivalent to part of our FMTL; Figure 16, "FTE-Bates") muscle's moment arm increased with knee extension in B.A.S.'s model, following a pattern similar to other knee extensors', but S.E.A. found a progressive decline of that muscle part's ("FMTM-Smith", Figure 16) knee extensor moment arm with knee extension. By comparing homologous muscles, it is evident that S.E.A.'s experimental data ("FMTM-Smith", Figure 16) match our model somewhat (FMTL; Figure 16; i.e. solid red and dashed blue lines toward the top of the upper right hand plot). Furthermore, the two distal parts of FMTL ("FTE" in Figure 16; B.A.S. and S.E.A. data) match each other somewhat. However, only our model represents the anatomy and function (weak knee flexion) of the actual medial head of M. femorotibialis (FMTM; Figure 16), which was not distinct in the data of B.A.S or S.E.A. Thus, a combination of incorrect classification of muscles (Appendix 1) and methodological differences explains divergence between some of the three studies' results. We noted similar problems with misidentifications (IFE, ITC, ITM muscles; Appendix 1) above in describing the results shown in Figure 10.

We raise the point here of the mistaken notion that tendon travel estimates of moment arms are free of errors in joint centre estimation, which is often repeated (e.g., Smith et al., 2007; Channon et al., 2010). This is only partly correct-- by using the virtual work principle that moment arms are equal to the change in musculotendinous length ("tendon travel") per unit of joint rotation, tendon travel experiments do minimize some errors in measuring moment arms, but still require joint centre estimates to calculate joint rotations, forming the traditional x-axis of moment arm vs. joint angle plots. Admittedly, all studies may carry this burden of error; our model is different in using empirically measured 3D joint axes. Yet typical tendon travel experiments still introduce another error, by introducing inevitable kinematic cross-talk between flexion/extension movements and motions about other planes (i.e. limb joints that are manually flexed/extended through a range of motion will also involve some motion in LAR and ab/adduction-- see Rubenson et al., 2007). Only the most rigorously constrained studies, which measure joint axes and constrain motions to strictly flexion-extension planes, avoid this problem. Models like ours and B.A.S.'s can explicitly avoid it. Our model's data show that, because moment arms of muscles co-vary with all joint angles (flex/extension, ab/adduction, long-axis 
765

766

767

768

769

770

771

772

773

774

775

776

777

778

779

780

781

782

783

784

785

786

787

788

789

790

791

792

793

794

795

796

797

798

799

800

801

802

803

804

805

806

807

808

809

810

rotation) (Figures 12-15, S1-S4), changes in multiple joint angles at once (i.e., kinematic crosstalk) will tend to produce different moment arms than changes in one angle at a time.

Bates and Schachner (2012:p.1342) inferred that, because their model's outputs matched experimental data, "predicted abduction/adduction and long-axis rotation moments are good estimates". We find some important differences between our results and those of either or both studies that somewhat weaken this inference (Figures 12-15). However, we reiterate and celebrate that all three studies discussed here obtain broadly similar results for most muscles, in particular emphasizing that moment arms are not constant for most muscles. Furthermore, Smith et al. (2007) and Bates and Schachner (2012) noted limitations similar to those mentioned above.

Some comparative data exist from studies of other ratites or more restricted datasets for ostriches. Hutchinson (2004a) provided estimates weighted by physiological cross-sectional area $\left(\mathrm{A}_{\text {phys }}\right)$ of "antigravity" (extensor) muscle moment arms for a $2 \mathrm{D}$ model of an ostrich, using the same specimen and hence congruent with our results. Alexander et al. (1979) created a simple 2D geometric model of an ostrich whose limb dimensions were similar to ours (cf. their Table I and our Table 1), obtaining comparable muscle moment arm measurements: femorotibialis $5.1 \mathrm{~cm}$ about the knee (ours $\sim 5 \mathrm{~cm}$ ), gastrocnemius $3.8 \mathrm{~cm}$ about the ankle (ours $\sim 4 \mathrm{~cm}$ ), and digital flexors $3.2 \mathrm{~cm}$ about the MTP joint (ours $\sim 2 \mathrm{~cm}$ ). This ostrich was $64 \%$ of the body mass of ours but had muscle masses about $85 \%$ of ours, helping to explain its $\sim 30 \%$ greater $\mathrm{A}_{\text {phys }}$ and $\mathrm{F}_{\max }$ values (in addition, surely, to shorter muscle fascicles; Equations 1,2), which are differences most likely attributable to its wild-caught status as opposed to our specimen's farm provenance.

Goetz et al. (2008) created a musculoskeletal model of an emu using a procedure grossly similar to ours, and obtained similar results - e.g. ILFB muscle having the largest hip extensor moment arm (but see Appendix 1). Troy et al. (2009) assumed that only the IFE, ITM, ITCR and PIFML muscles (homologous to ours) would resist hip adduction in their simplified emu model, but our analysis reveals that several more hip abductors exist, namely the IL, ILFB, FCM/L and CFP muscle groups (Table 4; Figures 14,15). See Lamas et al. (2014) for more consideration of emu pelvic limb muscle functions.

There are anatomical and methodological reasons to suggest that musculoskeletal modelling approaches (this study and Bates \& Schachner (2012)) can sometimes be more reliable than tendon-travel-based empirical data (e.g. Smith et al., 2007; also possibly Carr et al., 2011). First, the typical experimental method, as applied to date with birds, removes surrounding muscles and replaces whole 3D muscle bellies with quasi-2D strings (often lacking key "via points" or wrapping surfaces) that must alter the lines of action and thus moment arms of the in situ muscles. The potential for such alteration is experimentally testable, but existing studies of moment arms in various species (especially humans) already give strong precedent for this inference: analogous comparisons of "straight-line" 2D measurements (e.g., Jensen \& Davy, 1975) vs. 3D medical imaging or in situ measurements (e.g., Young et al., 1993; Arnold et al., 2001) vs. anatomically-realistic 3D musculoskeletal models (Delp et al., 1999; Arnold et al., 2000; Kargo \& Rome, 2002; Brown et al., 2003b; Burkholder \& Nichols, 2004; Blemker et al., 2006; O'Neill et al., 2013) show better agreement between the latter two methods, and areas where simple 2D measurements are imprecise or misleading. To be fair, poorly designed musculoskeletal models can have the same problems - faithful representations of anatomical geometry are vital for both methods.

Bates and Schachner (2012, their electronic supplementary material) note that their model obtained an "extremely close match to the experimental data, particularly given the level of intra- 
811 specific variation present in muscle moment arms for ostriches". Smith et al. (2007) did attribute

812 some of their experimental variation to individual differences in morphology that might alter

813 moment arms, but as they noted some of the variation may have instead been due to

814 experimental error. Young et al. (1993), using a rigorous, validated in situ experimental

815 apparatus for muscles crossing cat ankle joints, still found some individual variability that they

816 attributed to muscles tethered by soft tissues as opposed to those restricted by bony wrapping

817 surfaces or travelling in simple straight lines without any obstacles. We caution that these

818 different sources of error need to be weighed separately; individual variability is an interesting

819 biological reality, but experimental error needs minimizing. We suspect that some of the issues

820

821

822

823

824

825

826

827

828

829

830

831

832

833

834

835

836

837

838

839

840

841

842

843

844

845

846

847

848

849

850

851

852

853

854

855

856 raised above about unrealistic representation of musculoskeletal anatomy in tendon travel experiments might be due to experimental error. Bates and Schachner (2012) also raised the latter issue, finding that trends evident in Smith et al.'s (2007) moment arm data would leave the hip extensors too weak to balance the hip joint moments during stance phase, with a premature shift to hip flexor action. Our results (Figs. 9-20) indicate that tendon travel measurements of moment arms often exhibit steeper changes with joint angle in some cases, or constant moment arms where models show good reason to suspect variable moment arms with joint angle.

O'Neill et al.'s (2013; also Howlowka \& O'Neill, 2013) musculoskeletal modelling and experimental analysis of chimpanzee pelvic limb moment arms came to similar conclusions as we have here, concerning model vs. tendon travel estimates of moment arms (see also Brown et al., 2003a,b for horses). Importantly, they noted that non-linear trends in moment arm vs. joint angle data are to be expected, so tendon travel studies that enforce the fitting of largely linear (or simple polynomial) curves to their data may introduce errors. O'Neill et al. (2013) also cautioned that (p.3721) "the 3D orientation of the pelvis and thigh segments during tendon-excursion experiments can have a significant effect on moment arm metrics." Channon (2010) used cubic spline functions to characterize gibbon hindlimb muscle moment arms, presenting an improvement over the method used for ostriches by Smith et al. (2007) and considering some of the potential errors in tendon travel methods. Tightly controlled experiments with cats (Young et al., 1993) have shown good agreement with model-based moment arm data (Burkholder and Nichols, 2004).

Nonetheless, there is room for improvement in all methodologies. In particular, human experiments have shown that moment arms may depend on muscle activation levels (Maganaris, 2004; Tsaopoulos et al., 2007), a phenomenon that few moment arm studies have assimilated. Numerous studies have also shown that sarcomere or fibre length changes in real 3D muscles, as opposed to line segments, may be highly heterogeneous and complex (e.g., Blemker et al., 2005; Carr et al., 2011).

The accuracy of avian musculoskeletal models remains unresolved. Studies of moment arms for ostriches and other avian species badly need stronger validation tests - ideally $3 \mathrm{D}$, in vivo and across a range of behaviours - to test where different methods succeed or fail. O'Neill et al. (2013) noted that model-based estimates of moment arms should be less sensitive to the origins of muscles than to the insertions, and given that those insertions tend to be more easily circumscribed in ostriches (as in chimpanzees), a focus on improving the placement of muscle insertions could be powerful. At present, there is no published gold standard measurement that the aforementioned studies can be compared against, and thus it is unclear which estimates of moment arms are truly more accurate and reliable than others. Our judgements above might prove to be incorrect. We assume here, except where noted, that our moment arm estimates are generally an improvement over previous studies' because they are 3D, based on precise, subject- 
857 specific anatomical measurements of a single cadaver in situ, and incorporate modern data on the 858 3D complexity of avian limb joint axes. However, our assumption of improved accuracy 859 demands a test against a gold standard, with clear criteria for what a "good" agreement between

\section{Model assumptions and potential refinements}

Some simplifications of joint systems were necessary in our model but could be improved with later iterations. The tibio-fibular articulation is slightly mobile in ostriches (Fuss, 1996) and other birds, but we maintained it as an immobile joint. Likewise, the (proximal; see Chadwick et al., 2014; Regnault et al., 2014) patella surely translates (and perhaps rotates) during knee flexion/extension in birds as in humans (e.g. Walker et al., 1988; Suzuki et al., 2012) but we maintained it in the same resting position (with respect to the femur), represented simply by a wrapping surface. Adding such translation would influence the moment arm curves for knee extensor muscles. The intertarsal (ankle) joint's motions during swing phase (extreme dorsiflexion) seemed unrealistic, laterally rotating the tarsometatarsus to a seemingly disarticulated position (see Supplementary Movie S1), but we kept this as-is in the model rather than invent a subjective solution, as it would have minimal influence on our results here and maintained strict fidelity to our anatomical and kinematic data. Future implementations of the model emphasizing ankle joint mechanics (especially in the swing phase when dorsiflexion is prominent) may need to adjust these kinematics. The proximal interphalangeal joint of digit III was kept immobile in the model because our kinematic data lacked its angular motions, but the model has the capacity to allow the joint to flex and extend if desired (Table 1; Hutchinson et al., 2015), and could involve internal mechanisms such as those described by Schaller et al. (2011) if necessary for research questions addressed with it.

Our model's muscles were simplified, as the Methods and Supplementary Text explain. Our digitizing procedure, performed in 2002, was simplistic (similar to that of Burkholder and Nichols, 2004), whereas more recent techniques have fused CT and MRI imaging modalities to produce quite accurate and complex 3D musculoskeletal models (e.g., Zarucco et al., 2005, Harrison et al., 2014). Real muscles have complex 3D structure but we have simplified them into basic Hill model muscles of 2D structure. Internal tendons were observed in some muscles (e.g., M. iliotrochantericus caudalis, Mm. gastrocnemii, many digital flexors; Gangl et al., 2004). The Hill model does not discretely represent these features, which can affect muscle forces and gearing. Ligaments and other passive tissues were not represented in our model, and these would be particularly important features to consider in a complete dynamic model, as Haughton (1864) suggested and Schaller et al. (2009) demonstrated experimentally. Finally, a test of the validity of our moment arm estimates against a "gold standard" empirical measurement (see above) with explicit criteria for an acceptable level of precision would be valuable.

Yet like any model, simple or complex, our model is a useful starting point for a continually iterative process of improvement (enhanced by the open format of our model; Hutchinson et al., 2015) that progressively approximates reality. It has the advantage of being able to estimate muscle forces, moments, and length changes that are unlikely or impossible to be measurable in vivo, especially simultaneously across a full stride.

\section{Implications for ostrich limb muscle function}

Table 4 shows our classification of the major actions of ostrich pelvic limb muscles (also see Lamas et al. (2014) for emu muscles). This approach, as opposed to the classical perspective 
903 in comparative anatomy and biomechanics, emphasizes the three-dimensional nature of avian

904 limbs and the capacity of muscles to generate moments about many joints and degrees of

905 freedom (see also the categorization for cat ankle muscles provided by Young et al. (1993); also

906 model-based assessments using isometric force-fields by Kargo \& Rome (2002) and endpoint

907 forces by Burkholder \& Nichols (2004)). Standard functional anatomy papers tend to emphasize

908 flexion/extension and only present other actions (or any actions about other joints than those

909 deemed to be the main joints of action) in a seemingly arbitrary fashion (e.g., Smith et al., 2007:

910 table 2; cf. our Table 4), with at least the implicit assumption that non-parasagittal actions are

911 less important. Our approach also reveals how the functions of some muscles have been

912 misclassified before (or remain uncertain), for ostriches and perhaps for other avian species-- to

913 the degree that ostriches are representative of ancestral muscle functions, which remains to be

914 determined. The degree of uncertainty about avian pelvic limb muscle function particularly

915 applies to 3D actions about the hip joint; many muscles have actions (e.g., flexion/extension) that

916 switch depending on the joint angles adopted.

917 Examples of somewhat surprising 3D actions of hip muscles include the M. obturatorius

918 medialis (OM), which we find to mainly fulfill a lateral rotator and flexor action at the hip,

919 whereas Smith et al. (2006, 2007), Bates and Schachner (2012), Bates et al. (2012a,b) favour an

920 extensor action of this muscle (in addition to lateral rotation) in ostriches and other archosaurs. In

921 the model of B.A.S., this seems to be at least partly due to a distal insertion of the OM muscle on

922 the femur, rather than very proximally onto the trochanteric crest (Gangl et al., 2004; Zinoviev,

923 2005). Furthermore, some "antigravity muscles" act in one direction synergistically with other

924 muscles, resisting gravity, but in other direction(s) they act antagonistically, requiring greater

925 moments from other "antigravity" muscles. Clear examples include the PIFML and CFP

926 muscles, which are presumably active in hip extension but incur lateral rotator and abductor

927

928

929

930

931

932

933

934

935

936

937

938

939

940

941

942

943 moments about the hip (Table 4). These muscles probably oppose other antigravity muscles such as the ITC in medial rotation, whereas they would oppose potential hip extensors and adductors such as AMB1 (cf. moment data in Rubenson et al., 2011:fig. 7), indicating the complex dynamics of hip musculature.

The analysis of Rubenson et al. (2011:figure 7) indicates that hip adduction, not abduction, must be resisted during stance phase in locomoting ostriches, and thus abductor muscle activity is predicted, a moment that many hip extensors create anyway. However, hip adduction capacity is far more limited-- only the IC, AMB1, 2 and IFI muscles have clear actions in hip adduction. Smith et al. (2006:table 2) assigned adductor actions to other muscles such as the flexor cruris (FC) heads, PIFML and OM whereas we find these to be abductors. Indeed, the actions of the two heads of AMB may oppose each other (Table 4), so it would be interesting to know how they are coordinated. The ITC muscle's parts have clear roles in medial rotation, but their actions in flexion/extension vary with limb posture (see also Gatesy, 1994), rendering it less clear whether (or when) they play a predominant hip extensor (e.g. Rubenson et al., 2006) or hip flexor (e.g. Smith et al., 2006, 2007) role in ostriches or other birds. How any birds balance this complex interaction of long-axis and ab/adductor moments at the hip or other joints remains

944

945

946

947 almost unexplored (but see Gatesy 1994), yet modelling (and simulation) approaches such as ours offer one way to tease apart the complexity. Bates and Schachner (2012) found that Alligator and Struthio had similar hip extensor moment arms but there were large abduction and small adduction moment arms in their ostrich, along with large long-axis rotation moment arms.

948 The functional and evolutionary implications of these differences remain unclear, and dependent on understanding force balance about the hip joint in extant archosaurs such as Struthio. 
949

950

951

952

953

954

955

956

957

958

959

960

961

962

963

964

965

966

967

968

969

970

971

972

973

974

975

976

977

978

979

980

981

982

983

984

985

986

987

988

989

990

991

992

993

994

Complex function is not restricted to proximal muscles, however. Complex anatomy of distal limb muscles is a pernicious problem in avian locomotor biomechanics, and difficult to render realistically in biomechanical models such as ours. As the Supplementary Text describes, we could not model all origins (or subdivided tendons; e.g., digital flexors) of all muscle parts. Indeed, in some cases the origins are diffuse - e.g. M. gastrocnemius medialis originates mainly from the medial side of the proximal tibiotarsus, but the surrounding fascia it is attached to continue proximally past the knee joint, via the proximal patella and other structures. It is not clear if some of these distal muscles exert important moments about the knee joint (some forces may be going directly to their distal origins from the tibiotarsus), and the dynamics of the patella (not represented in our model except as a static wrapping surface) further complicates matters. Thus it is unclear how forces are balanced across ostrich (or other avian) knees, complicating comparisons with other species (e.g. Higham et al., 2008; Andrada et al., 2013).

Young et al. (1993) and Johnson et al. (2012) noted that some muscles in cat and rat hindlimbs seemed to have intrinsically stabilizing properties, shifting from flexor to extensor moment arms in a linear fashion with increasing joint flexion. Eight modelled ostrich limb muscles also show this pattern: the AMB1, AMB2, IC, ITCa, ITCp, ITM, ITCR and ISF exhibit stabilization function in flexion-extension (Figures 9,10). Weaker evidence for self-stabilization is present for the OM muscle in hip ab/adduction (Figure 14) and the four ankle flexors in flexion/extension (TCf, TCt, EDL, and FL; Figure 18), so any self-stabilization properties must be interpreted as being largely restricted to the hip's flexion-extension function (see also Table 4). Judging from these hip muscles' paths, their long lines of action (due to the elongate pelvis and limb) running close to the hip seem to predispose them to these intrinsic stabilization properties. These patterns deserve more examination in a comparative context with other species in the future -- for example, similar hip muscles show similar moment arm patterns that hint at intrinsic stabilization in various extinct dinosaurs and other archosaurian reptiles (Hutchinson et al., 2005,2008; Bates and Schachner, 2012; Bates et al, 2012a,b; Maidment et al., 2013).

\section{Musculoskeletal models of limb function: past, present and future}

A wide variety of studies have used musculoskeletal models to reconstruct limb function in extant and extinct animals, but there remains little agreement for standards of model design, analysis and validation. The same software (SIMM) or other packages (GaitSym, Anybody, varieties of Adams, etc.) has been used to estimate limb muscle moment arms in other extant species including chimpanzees and other hominins (O'Neill et al., 2013; Holowka and O'Neill, 2013; and references therein), horses (Brown et al., 2003a,b; Zarucco et al., 2006; Harrison et al., 2010), domestic cats (Burkholder \& Nichols, 2004), rats (Johnson et al., 2008), emus (Goetz et al., 2008), Alligator and ostriches (Bates and Schachner, 2012), frogs (Kargo and Rome, 2002; Kargo et al., 2002), cockroaches (Full \& Ahn, 1995) and others, in addition to simpler past approaches (e.g. for small mammals, McClearn, 1985). It is not clear which software packages most accurately estimate muscle moment arms, but our comparisons with the data from Bates and Schachner (2012) suggest some advantages of our approach. Regardless, comparative biologists seeking to harness the power of musculoskeletal modelling techniques have much to learn from the considerable progress made in analyzing and validating similar models of human limb muscles (e.g., Delp et al., 1990,1992,1999; Arnold et al., 2000,2001; Holzbauer et al., 2005; Nikooyan et al., 2011).

There has been a recent flourishing of musculoskeletal models of extinct taxa, especially hominins (e.g., Australopithecus in Nagano et al., 2005) and dinosaurs or other archosaurs. 
995 Theropod dinosaurs have featured prominently, including Allosaurus, Struthiomimus (Bates and

996 Schachner, 2012; Bates et al. 2012a), Tyrannosaurus (Hutchinson et al., 2005), and Velociraptor

997 (Hutchinson et al., 2008), among other taxa (Allen, 2010). More recently, sauropodomorph

998 (Mallison, 2010a,b) as well as ornithischian dinosaurs have enjoyed a new focus (Mallison,

999 2010c; Bates et al., 2012b; Fujiwara and Hutchinson, 2012; Maidment et al., 2013). Other extinct

1000 taxa studied, for example, includethe stem-crocodile Poposaurus (Bates and Schachner, 2012)

1001 and related taxa (Molnar, 2014), a pterosaur (Costa et al., 2013) and the early tetrapod

1002 Ichthyostega (Pierce et al., 2012). The detail and apparent effort put into these models varies

1003 widely, often depending on the research questions being asked. It remains debatable how

1004 detailed such models of extinct taxa need to be in order to be sufficiently accurate - are cruder

1005 models good enough for strictly comparative work? There are clear analogies with the usage of

1006 finite element models (e.g. Rayfield, 2007) —often, it is argued that simpler models are

1007 sufficient for simple comparative questions, whereas more complex questions such as bone

1008 failure (or, for musculoskeletal models, locomotor dynamics or performance?) require more

1009 realistic models.

1010 Whether the focus is on limb joint ranges of motion, muscle moment arms, or dynamic

1011 simulation, there is a need for more scrutiny of models of extant taxa to establish how accurate

1012 and reliable these modelling approaches truly are, and ultimately a renewed examination and

1013 synthesis of those findings with past studies of extinct taxa. Studies using models to estimate

1014 muscle moment arms need to consider not only this, but also how or whether those parameters

1015 actually matter for particular muscles, joints, behaviours or species (see also Bates and

1016 Schachner, 2012; Bates et al., 2012b; Maidment et al., 2013).

1017 To the degree that general principles of moment arm usage exist, they should clarify

1018 under what circumstances a moment arm is optimized to perform a certain function. An

1019 understanding of this link between muscle mechanics, kinematics and moment arms could link

1020 the disparate palaeobiological/comparative studies on limb joint ranges of motion and

1021 orientations (e.g., Mallison, 2010a,b,c; Pierce et al., 2012; Molnar, 2014) with those of limb

1022 muscle moment arms (Hutchinson et al., 2005,2008; Allen, 2010; Bates and Schachner, 2012;

1023 Bates et al., 2012b; Fujiwara and Hutchinson, 2012; Maidment et al., 2013). Such a synthesis

1024 could lead to a robust, sustainable future for comparative musculoskeletal modelling and

1025 simulation, and new insights into the evolution of musculoskeletal function and locomotor

1026 dynamics.

1027

1028

1029

1030

1031

1032

1033

1034

1035

1036

1037

1038

1039

1040

\section{Acknowledgments}

We thank Peter Loan for kind and patient technical support. Thanks to Aaron Daub for his proficient assistance in the ostrich digitization and dissection. This paper benefited from discussions with F. Clay Anderson, Allison Arnold, Silvia Blemker, Steve Gatesy, and Karl Bates, as well as with members of the Stanford Neuromuscular Biomechanics Laboratory and the Structure \& Motion Laboratory at The Royal Veterinary College. We graciously appreciate constructive reviews by Robert Kambic and Karl Bates, and comments from Diego Sustaita, that improved the manuscript.

\section{APPENDIX 1:}


1041

1042

1043

1044

1045

1046

1047

1048

1049

1050

1051

1052

1053

1054

1055

1056

1057

1058

1059

1060

1061

1062

1063

1064

1065

1066

1067

1068

1069

1070

1071

1072

1073

1074

1075

1076

1077

1078

1079

1080

1081

1082

1083

1084

1085

1086
A few observations from our dissections revealed differences in muscle identifications from previous studies, which themselves have had much disagreement (reviewed in Gangl et al., 2004; Zinoviev, 2006). However, generally our findings agree well with the excellent study by Gangl et al. (2004). Zinoviev (2006) made some amendments to the latter study that our dissections independently have confirmed.

First, rather than treat "M. pectineus" as a novel muscle unrelated to M. ambiens (e.g., Mellett, 1994; Gangl et al., 2004) or as a part of M. femorotibialis medialis (= FMTM or "internus"; Zinoviev, 2006), we refer to the muscle here as M. ambiens 1 (AMB1; for its pubic origin as in most other Reptilia; Hutchinson, 2002) and refer to the muscle with the derived iliac origin as M. ambiens 2 (AMB2; i.e., the dorsal head) (Table 2). We do not intend for this to be a formal nomenclatural change but it is used for convenience within this paper and to suggest that the homologies (and thus formal nomenclature) of these two muscle heads deserve reconsideration. Our nomenclature avoids confusion with the mammalian M. pectineus which has no homology with these muscles, and maintains the same nomenclature for the homologous M. ambiens (whether one or two parts) in extant Sauropsida. We deem these homologies and nomenclature marginally more parsimonious than other interpretations (reviewed by Zinoviev, 2006), the latest of which (favoured by Zinoviev, 2006) requires loss of the origin of M. ambiens from the pectineal (preacetabular) process that is plesiomorphically present in birds (Hutchinson, 2002) and gain of a new head of the FMTM muscle (the "pars pectineus"). In our scenario, mainly a new head of M. ambiens (AMB2) is required; a phenomenon that is not unknown in other birds and is pervasive in Crocodylia (Hutchinson, 2002).

Second, we agree with Zinoviev (2006) that Gangl et al. (2004) confused the two crura of "M. iliofemoralis externus" with M. iliotrochantericus caudalis, which engendered further errors in identifying other muscles. We have confirmed this from dissections of three additional ostrich cadavers, and hence the position of our M. iliotrochantericus caudalis (ITCa,ITCp in Table 2) differs from theirs (also data in Smith et al., 2006,2007). We consider M. iliofemoralis externus (IFE) to be weakly differentiated from the latter muscle, also intimated by Gangl et al. (2004:p.113; and Gadow, 1880), but we represent it in our model as a separate head (IFE), again agreeing with Zinoviev (2006). This is corroborated by the main muscle in question (ITC) having a large internal tendon, preacetabular position (between the heads of Mm. iliotibiales; IC and IL), and overlaying Mm. iliotrochanterici medius et cranialis (ITM, ITCR), which are traits diagnostic of M. iliotrochantericus caudalis, not "M. iliofemoralis externus" as in Gangl et al. (2004). This then explains why the latter study did not find M. iliotrochantericus medius - it identified it as M. iliotrochantericus caudalis, noting some fusion and distolateral insertion with M. iliotrochantericus cranialis, which are diagnostic of M. iliotrochantericus medius instead. Hence our M. iliotrochantericus medius (ITM) corresponds to their "M. iliotrochantericus caudalis"; but our M. iliotrochantericus cranialis (ITCR) corresponds to theirs. Smith et al. (2006,2006) perpetuated the errors caused by Gangl et al.'s misidentifications, which complicates comparisons with our data (see our Results section, Fig. 10). Bates and Schachner (2012) appear to have avoided these errors.

A third point of discordance between this study and Gangl et al.'s (2004) is that we consider the latter study's "Mm. femorotibiales externus et medius" to be two parts (superficial and deep) of M. femorotibialis lateralis (FMTL; vide Zinoviev, 2006), because this avian muscle typically originates on the lateral surface of the femur, deep to M. iliotibialis lateralis (IL), as the former two parts do. Gangl et al.'s (2004) “Mm. femorotibiales internus et accessorius” then correspond to our Mm. femorotibiales medialis et intermedius (FMTM, FMTIM), because their 
1087 topological connections more closely match these muscles in birds. Zinoviev (2006) again gave a 1088 detailed correction that we concur with, whereas Smith et al. $(2006,2007)$ and possibly Bates 1089 and Schachner (2012) used Gangl et al.'s (2004) misidentifications. Accepting these identifications renders ostrich anatomy more similar to other birds: M. iliotrochantericus caudalis remains large and originating dorsal to $\mathrm{M}$. iliotrochantericus medius, which originates cranial to M. iliotrochantericus cranialis, and the insertions of these muscles retain similar proximodistal positions (the first on the lateral surface of the proximal femur, the second two sequentially distal on the craniolateral edge of the trochanteric crest; Rowe, 1986; Hutchinson, 2002). Furthermore, Mm. femorotibiales in our view then match avian anatomical positions more reasonably.

However, ostrich pelvic muscle anatomy is still derived in the large relative size of M. iliofemoralis internus (IFI; "cuppedicus" of Rowe, 1986; Table 2) and the two distinct heads of M. ambiens (i.e., the novel dorsal head, AMB2). Additionally, M. iliotrochantericus medius (ITM) is slightly derived in having a markedly more dorsal position (required by the dorsally shifted M. ambiens and M. iliofemoralis internus (IFI)) than in typical neornithines, and Mm. femorotibiales have strong division of the lateral head (FMTL) into two parts, but not a novel "accessorius" head. Furthermore, M. obturatorius medialis $(\mathrm{OM})$ in ostriches is extraordinary in its mostly lateral origin, whereas normally in birds it is confined to the inside of the pelvis. Similar problems with muscle identifications vex other studies of ratite limb form and function. Patak \& Baldwin's (1998) anatomical description of emu pelvic limb muscles was used to formulate Goetz et al.'s (2008) musculoskeletal model of an emu, incorporating analogous errors that deserve correction in future studies (see Lamas et al., 2014). The issues outlined in this appendix drive home the point that a firm grasp of avian myology, ideally based upon understanding of fundamental groups of muscles likely present in the ancestral neornithine bird and hence homologous among its living descendants, is critical for any biomechanical and comparative analyses of muscle form, function and evolution (see also Zinoviev, 2006). 


\section{References}

1114

1115

1116

1117

1118

1119

1120

1121

1122

1123

1124

1125

1126

1127

1128

1129

1130

1131

1132

1133

1134

1135

1136

1137

1138

1139

1140

1141

1142

1143

1144

1145

1146

1147

1148

1149

1150

1151

1152

1153

1154

1155

1156

1157

1158
Abourachid, A. 2001. Kinematic parameters of terrestrial locomotion in cursorial (ratites), swimming (ducks), and striding birds (quail and guinea fowl). Comparative Biochemistry and Physiology A 131:113-119.

Abourachid, A., \& Renous, S. 2000. Bipedal locomotion in ratites (Paleognatiform) [sic]: examples of cursorial birds. Ibis 142:538-549.

Abourachid, A, Hackert, R., Herbin, M., Libourel, P. A., Lambert, F., Gioanni, H., Provini, P., Blazevic, P., \& Hugel, V. 2011. Bird terrestrial locomotion as revealed by 3D kinematics. Zoology 114: 360-368.

Alexander R. McN, Maloiy, G.M.O., Njau, R., \& Jayes, A.S. 1979. Mechanics of running of the ostrich (Struthio camelus). Journal of Zoology 187:169-178.

Allen, V. 2010. The evolution of avian hindlimb conformation and locomotor function. Unpublished PhD dissertation, The Royal Veterinary College, University of London, United Kingdom.

Andrada, E., Nyakatura, J. A., Bergmann, F., \& Blickhan, R. 2013. Adjustments of global and local hindlimb properties during terrestrial locomotion of the common quail (Coturnix coturnix). Journal of Experimental Biology 216: 3906-3916.

Arnold, A. S., \& Delp, S. L. 2001. Rotational moment arms of the medial hamstrings and adductors vary with femoral geometry and limb position: implications for the treatment of internally rotated gait. Journal of Biomechanics 34:437-447.

Arnold, A. S., Salinas, S., Asakawa, D. J., \& Delp, S. L. 2000. Accuracy of muscle moment arms estimated from MRI-based musculoskeletal models of the lower extremity. Computer Aided Surgery 5:108-119.

Baker, A.J., Haddrath, O., McPherson, J. R., \& Cloutier, A. 2014. Genomic support for a moatinamou clade and adaptive morphological convergence in flightless ratites. Molecular Biology and Evolution, in press.

Bates, K. T., \& Schachner, E. R. 2012. Disparity and convergence in bipedal archosaur locomotion. Journal of the Royal Society Interface 9: 1339-1353.

Bates, K. T., Benson, R. B., \& Falkingham, P. L. 2012a. A computational analysis of locomotor anatomy and body mass evolution in Allosauroidea (Dinosauria: Theropoda). Paleobiology 38: 486-507.

Bates, K. T., Maidment, S. C., Allen, V., \& Barrett, P. M. 2012b. Computational modelling of locomotor muscle moment arms in the basal dinosaur Lesothosaurus diagnosticus: assessing convergence between birds and basal ornithischians. Journal of Anatomy 220: 212-232. 
Bennett, M. B., \& Taylor, G. C. 1995. Scaling of elastic strain energy in kangaroos and the benefits of being big. Nature 378: 56-59.

Biewener, A. A. 1989. Scaling body support in mammals: limb posture and muscle mechanics. Science 245:45-48.

Biewener, A. A. 1990. Biomechanics of mammalian terrestrial locomotion. Science 250:10971103.

Blemker, S. S., Pinsky, P. M., \& Delp, S. L. 2005. A 3D model of muscle reveals the causes of nonuniform strains in the biceps brachii. Journal of Biomechanics 38: 657-665.

Bottema, O., \& Roth, B. 1990. Theoretical Kinematics. Vol. 1. Dover Publications.

Brassey, C. A., Kitchener, A. C., Withers, P. J., Manning, P. L., \& Sellers, W. I. 2013a. The role of cross-sectional geometry, curvature, and limb posture in maintaining equal safety factors: A computed tomography study. Anatomical Record 296: 395-413.

Brassey, C. A., Holdaway, R. N., Packham, A. G., Anné, J., Manning, P. L., \& Sellers, W. I. 2013b. More than one way of being a moa: Differences in leg bone robustness map divergent evolutionary trajectories in Dinornithidae and Emeidae (Dinornithiformes). PLoS one, 8(12): e82668.

Brown, N. A. T., Pandy, M. G., Buford, W. L., Kawcak, C. E., \& McIlwraith, C. W. 2003 a. Moment arms about the carpal and metacarpophalangeal joints for flexor and extensor muscles in equine forelimbs. American Journal of Veterinary Research 64:351-357.

Brown, N. A. T., Pandy, M. G., Kawcak, C. E., \& McIlwraith, C. W. 2003b. Force- and momentgenerating capacities of muscles in the distal forelimb of the horse. Journal of Anatomy 203:101113.

Burkholder, T. J., \& Nichols, T. R. 2004. Three-dimensional model of the feline hindlimb. Journal of Morphology 261: 118-129.

Cappozzo, A., Catani, F., Della Croce, F., \& Leardini, A. 1995. Position and orientation in space of bones during movement: anatomical frame definition and determination. Clin. Biomech. 10, 171-178.

1204

Carr, J. A., Ellerby, D. J., Rubenson, J., \& Marsh, R. L. 2011. Mechanisms producing coordinated function across the breadth of a large biarticular thigh muscle. Journal of Experimental Biology 214: 3396-3404.

Chadwick, K.P., Regnault, S., Allen, V., \& Hutchinson, J.R. 2014. Three-dimensional anatomy of the ostrich (Struthio camelus) knee joint. PeerJ 2:e706 http://dx.doi.org/10.7717/peerj.706 
1205 Channon, A. J., Crompton, R. H., Günther, M. M., \& Vereecke, E. E. 2010. Muscle moment 1206 arms of the gibbon hind limb: implications for hylobatid locomotion. Journal of Anatomy 216: 1207 446-462.

Costa, F. R., Rocha-Barbosa, O., \& Kellner, A. W. A. 2013. A biomechanical approach on the optimal stance of Anhanguera piscator (Pterodactyloidea) and its implications for pterosaur gait on land. Historical Biology, (ahead-of-print), 1-9.

\section{Cracraft, J., \& Clarke, J. 2001. The basal clades of modern birds. In: Gauthier JA, Gall LF,} editors. New perspectives on the origin and early evolution of birds: proceedings of the international symposium in honor of John H. Ostrom. New Haven, CT: Peabody Mus Nat Hist. p 143-156.

Daley, M. A., \& Usherwood, J. R. 2010. Two explanations for the compliant running paradox: reduced work of bouncing viscera and increased stability in uneven terrain. Biology Letters 6: 418-421.

Delp, S. L., \& Zajac, F. E. 1992. Force- and moment-generating capacity of lower-extremity muscles before and after tendon lengthening. Clinical Orthopaedics 284:247-259.

Delp, S. L., \& Loan, J. P. 1995. A graphics-based software system to develop and analyze models of musculoskeletal structures. Computers in Biology and Medicine 25:21-34.

Delp, S. L., \& Loan, J. P. 2000. A computational framework for simulating and analyzing human and animal movement. IEEE Computing in Science and Engineering 2:46-55.

Delp, S. L., Loan, J. P., Hoy, M. G., Zajac, F. E., Topp, E. L., \& Rosen, J. M. 1990. An interactive graphics-based model of the lower extremity to study orthopaedic surgical procedures. IEEE Transactions in Biomedical Engineering 37:757-767.

Delp, S. L., Hess, W. E., Hungerford, D. S., \& Jones, L. C. 1999. Variation of rotation moment arms with hip flexion. Journal of Biomechanics 32:493-501.

Fedak, M. A. \& Seeherman, H. J.. 1979. Reappraisal of energetics of locomotion shows identical cost in bipeds and quadrupeds including ostrich and horse. Nature 282:713-716.

Fedak, M. A., Heglund, N. C., \& C. R. Taylor. 1982. Energetics and mechanics of terrestrial locomotion. II. Kinetic energy changes of the limbs and body as a function of speed and body size in birds and mammals. Journal of Experimental Biology 79:23-40.

Fujiwara, S. I. 2009. Olecranon orientation as an indicator of elbow joint angle in the stance phase, and estimation of forelimb posture in extinct quadruped animals. Journal of Morphology 270: $1107-1121$. 
1249 Fujiwara, S. I., \& Hutchinson, J. R. 2012. Elbow joint adductor moment arm as an indicator of 1250 forelimb posture in extinct quadrupedal tetrapods. Proceedings of the Royal Society B:

1251 Biological Sciences 279: 2561-2570.

1252

1253

1254

1255

1256

1257

1258

1259

1260

1261

1262

1263

1264

1265

1266

1267

1268

1269

1270

1271

1272

1273

1274

1275

1276

1277

1278

1279

1280

1281

1282

1283

1284

1285

1286

1287

1288

1289

1290

1291

1292

1293

1294

Fujiwara, S. I., Endo, H., \& Hutchinson, J. R. 2011. Topsy-turvy locomotion: biomechanical specializations of the elbow in suspended quadrupeds reflect inverted gravitational constraints. Journal of Anatomy 219: 176-191.

Full, R., \& Ahn, A. 1995. Static forces and moments generated in the insect leg: comparison of a three-dimensional musculo-skeletal computer model with experimental measurements. Journal of Experimental Biology 198: 1285-1298.

Fuss, F. K. 1996. Tibiofibular junction of the South African ostrich (Struthio camelus australis). Journal of Morphology 227:213-226.

Gadow, H. 1880. Zur vergleichenden Anatomie der Muskulatur des Beckens und der hinteren Gliedmasse der Ratiten. Jena, G. Fischer.

Gangl D., Weissengruber, G.E., Egerbacher, M., \& Forstenpointer, G. 2004. Anatomical description of the muscles of the pelvic limb in the ostrich (Struthio camelus). Anatomia Histologia Embryologia 33:100-114.

Gatesy S. M. 1990. Caudofemoral musculature and the evolution of theropod locomotion. Paleobiology 16:170-186.

Gatesy, S. M. 1994. Neuromuscular diversity in archosaur deep dorsal thigh muscles. Brain Behavior and Evolution, 43: 1-14.

Gatesy, S.M. 1995. Functional evolution of the hindlimb and tail from basal theropods to birds. Pp. 219-234 in Thomason, J.J. (ed.), Functional Morphology in Vertebrate Paleontology. Cambridge University Press, UK.

Gatesy, S.M., \& Biewener, A.A. 1991. Bipedal locomotion - effects of speed, size and limb posture in birds and humans. Journal of Zoology 224:127-147.

Gatesy, S. M., Bäker, M., \& Hutchinson, J. R. 2009. Constraint-based exclusion of limb poses for reconstructing theropod dinosaur locomotion. Journal of Vertebrate Paleontology 29: 535544.

Goetz, J. E., Derrick, T. R., Pedersen, D. R., Robinson, D. A., Conzemius, M. G., Baer, T. E., \& Brown, T. D. 2008. Hip joint contact force in the emu (Dromaius novaehollandiae) during normal level walking. Journal of Biomechanics 41: 770-778.

Grood, E. S., \& Suntay, W. J. 1983. A joint coordinate system for the clinical description of three-dimensional motions: application to the knee. Journal of Biomechanical Engineering 105, $136-44$.

1295 
1296 Günther, M., Keppler, V., Seyfarth, A., \& R. Blickhan. 2004. Human leg design: optimal axial 1297 alignment under constraints. Journal of Mathematical Biology 48:623-646.

1298

1299

1300

1301

1302

1303

1304

1305

1306

1307

1308

1309

1310

1311

1312

1313

1314

1315

1316

1317

1318

1319

1320

1321

1322

1323

1324

1325

1326

1327

1328

1329

1330

1331

1332

1333

1334

1335

1336

1337

1338

1339

1340

1341
Haughton, R. 1864. On the muscular mechanism of the leg of the ostrich. Annals \& Magazine of Natural History $3^{\text {rd }}$ Series 15:262-272.

Haughton, R. 1867a. Notes on animal mechanics. No. X. Muscular anatomy of the emu (Dromaeus novae hollandiae). Proceedings of the Irish Academy of Science 9:487-497.

Haughton, R. 1867b. Notes on animal mechanics. No. XI. Muscular anatomy of the rhea (Struthio rhea). Proceedings of the Irish Academy of Science 9:497-504.

Higham, T. E., Biewener, A. A., \& Wakeling, J. M. 2008. Functional diversification within and between muscle synergists during locomotion. Biology Letters 4: 41-44.

Holowka, N. B., \& O'Neill, M. C. 2013. Three-dimensional moment arms and architecture of chimpanzee (Pan troglodytes) leg musculature. Journal of Anatomy 223: 610-628.

Holzbaur, K.R., Murray, W.M. \& Delp, S.L. 2005. A model of the upper extremity for simulating musculoskeletal surgery and analyzing neuromuscular control. Annals of Biomedical Engineering 33:829-840.

Hutchinson, J.R. 2002. The evolution of hindlimb tendons and muscles on the line to crowngroup birds. Comparative Biochemistry and Physiology A 133:1051-1086.

Hutchinson, J. R. 2004. Biomechanical modeling and sensitivity analysis of bipedal running ability. I. Extant taxa. Journal of Morphology 262:421-440.

Hutchinson, J. R., \& Allen, V. 2009. The evolutionary continuum of limb function from early theropods to birds. Naturwissenschaften 96: 423-448.

Hutchinson, J. R., \& Gatesy, S. M. 2000. Adductors, abductors, and the evolution of archosaur locomotion. Paleobiology 26: 734-751.

Hutchinson, J.R., Anderson, F.C., Blemker, S.S., \& Delp, S.L. 2005. Analysis of hindlimb muscle moment arms in Tyrannosaurus rex using a three-dimensional musculoskeletal computer model. Paleobiology 31:676-701.

Hutchinson, J. R., Miller, C., Fritsch, G., \& Hildebrandt, T. 2008. The anatomical foundation for multidisciplinary studies of animal limb function: examples from dinosaur and elephant limb imaging studies. In: Anatomical Imaging (eds. Fritsch, G. and Frey, R.), pp. 23-38. Springer, Japan.

Hutchinson, J.R., Rankin, J.W., Rubenson, J., Rosenbluth, K.H., Siston, R.A., Delp, S.L. 2015. Ostrich biomechanical computer model files and code. Figshare.

http://dx.doi.org/10.6084/m9.figshare. 1409443 
Jensen, R. H., \& Davy, D. T. 1975. An investigation of muscle lines of action about the hip: A 1345

Jindrich, D. L., Smith, N. C., Jespers, K., \& Wilson, A. M. 2007. Mechanics of cutting maneuvers by ostriches (Struthio camelus). Journal of Experimental Biology, 210: 1378-1390.

Johnson, W. L., Jindrich, D. L., Roy, R. R., \& Edgerton, V. R. 2008. A three-dimensional model of the rat hindlimb: musculoskeletal geometry and muscle moment arms. Journal of Biomechanics 41: 610-619.

Kambic, R. E., Roberts, T. J., \& Gatesy, S. M. 2014. Long-axis rotation: a missing degree of freedom in avian bipedal locomotion. Journal of Experimental Biology, jeb-101428; published online.

Kargo, W. J., \& Rome, L. C. 2002. Functional morphology of proximal hindlimb muscles in the frog Rana pipiens. Journal of Experimental Biology 205:1987-2004.

Kargo, W. J., Nelson, F. \& Rome, L. C. 2002. Jumping in frogs: assessing the design of the skeletal system by anatomically realistic modeling and forward dynamic simulation. Journal of Experimental Biology 205:1683-1702.

Kilbourne, B. M. 2013. On birds: scale effects in the neognath hindlimb and differences in the gross morphology of wings and hindlimbs. Biological Journal of the Linnean Society, 110: 1431 .

Lamas, L., Main, R.P., Hutchinson, J.R. 2014. Ontogenetic scaling patterns and functional anatomy of the pelvic limb musculature in emus (Dromaius novaehollandiae). PeerJ 2:e716 http://dx.doi.org/10.7717/peerj.716

Lieber, R. L., \& Boakes, J. L. 1988a. Sarcomere length and joint kinematics during torque production in frog hindlimb. American Journal of Physiology 254: C759-C768.

Lieber, R. L., \& Boakes, J. L. 1988b. Muscle force and moment arm contributions to torque production in frog hindlimb. American Journal of Physiology 254: C769-72.

Lieber, R. L., \& Brown, C. G. 1992. Sarcomere length-joint angle relationships of seven frog hindlimb muscles. Cells Tissues Organs 145: 289-295.

Lieber, R. L., \& Shoemaker, S. D. 1992. Muscle, joint, and tendon contributions to the torque profile of frog hip joint. American Journal of Physiology 263: R586-R586.

Lieber, R. L. 1997. Muscle fiber length and moment arm coordination during dorsi- and plantarflexion in the mouse hindlimb. Acta Anatomica 159:84-89.

Macalister, A. 1864. On the anatomy of the ostrich (Struthio camelus). Proceedings of the Royal Irish Academy 9: 1-24. 
Mai, M. T., \& Lieber, R. L. 1990. A model of semitendinosus muscle sarcomere length, knee and hip joint interaction in the frog hindlimb. Journal of Biomechanics 23: 271-279.

Maganaris, C. N. 2004. Imaging-based estimates of moment arm length in intact human muscletendons. European Journal of Applied Physiology 91:130-139.

Maidment, S. C., Bates, K. T., Falkingham, P. L., VanBuren, C., Arbour, V., \& Barrett, P. M. 2013. Locomotion in ornithischian dinosaurs: an assessment using three-dimensional computational modelling. Biological Reviews, published online. DOI: 10.1111/brv.12071

Mallison, H. 2010a. The digital Plateosaurus I: body mass, mass distribution, and posture assessed using CAD and CAE on a digitally mounted complete skeleton. Palaeontologia Electronica 13(13.2).

Mallison, H. 2010b. The digital Plateosaurus II: An assessment of the range of motion of the limbs and vertebral column and of previous reconstructions using a digital skeletal mount. Acta Palaeontologica Polonica, 55: 433-458.

Mallison, H. 2010c. CAD assessment of the posture and range of motion of Kentrosaurus aethiopicus Hennig 1915. Swiss Journal of Geosciences, 103: 211-233.

Maloiy, G. M. O., R. Mc N. Alexander, R. Njau, and A. S. Jayes. 1979. Allometry of the legs of running birds. Journal of Zoology 187:161-167.

McClearn, D. 1985. Anatomy of raccoon (Procyon lotor) and coati (Nasua narica and N. nasua) forearm and leg muscles: Relations between fiber length, moment-arm length, and joint-angle excursion. Journal of Morphology 183: 87-115.

McGowan, C. 1979. The hind limb musculature of the Brown Kiwi, Apteryx australis mantelli. Journal of Morphology 160:33-74.

Medler, S. 2002. Comparative trends in shortening velocity and force production in skeletal muscle. Am J Physiol Regulat Integr Comp Physiol 283:R368-R378.

Mendez, J., Keys, A. 1960. Density and composition of mammalian muscle. MetabolismClinical and Experimental 9:184-188.

Molnar, J. 2014. The biomechanics of vertebrae across evolutionary transitions between water and land: examples from early Tetrapod and Crocodylomorpha. Unpublished $\mathrm{PhD}$ dissertation, The Royal Veterinary College, University of London, United Kingdom.

Nagano, A., Umberger, B. R., Marzke, M. W. \& Gerritsen, K. G. M. 2005.

Neuromusculoskeletal computer modeling and simulation of upright, straight-legged, bipedal locomotion of Australopithecus afarensis. American Journal of Physical Anthropology 126: 2143313. 
Nelson, F. E., Gabaldón, A. M., \& Roberts, T. J. 2004. Force-velocity properties of two avian 1437

Nikooyan, A. A., Veeger, H. E. J., Chadwick, E. K. J., Praagman, M. \& van der Helm, F. C. T. 2011. Development of a comprehensive musculoskeletal model of the shoulder and elbow. Medical \& Biological Engineering \& Computing 49:1425-1435.

O'Neill, M. C., Lee, L. F., Larson, S. G., Demes, B., Stern, J. T., \& Umberger, B. R. 2013. A three-dimensional musculoskeletal model of the chimpanzee (Pan troglodytes) pelvis and hind limb. Journal of Experimental Biology 216:3709-3723.

Pandy, M. G. 1999. Moment arm of a muscle force. Exercise and Sport Science Reviews 27:79118.

Patak, A., \& Baldwin, J. 1993. Structural and metabolic characterization of the muscles used to power running in the emu (Dromaius novaehollandiae), a giant flightless bird. Journal of Experimental Biology 175:233-249.

Patak, A., \& Baldwin, J. 1998. Pelvic limb musculature in the emu Dromaius novaehollandiae (Aves: Struthioniformes: Dromaiidae): Adaptations to high-speed running. Journal of Morphology 238:23-37.

Paul, G.S. 1998. Limb design, function and running performance in ostrich-mimics and tyrannosaurs. Gaia 15:257-270.

Picasso, M. B. J. 2010. The hindlimb muscles of Rhea americana (Aves, Palaeognathae, Rheidae). Anatomia Histologia Embryologia 39: 462-472.

Picasso, M. B. J. 2012. Postnatal ontogeny of the locomotor skeleton of a cursorial bird: greater rhea. Journal of Zoology 286: 303-311.

Pierce, S. E., Clack, J. A., \& Hutchinson, J. R. 2012. Three-dimensional limb joint mobility in the early tetrapod Ichthyostega. Nature 486: 523-526.

Rayfield, E. J. 2007. Finite element analysis and understanding the biomechanics and evolution of living and fossil organisms. Annual Review of Earth and Planetary Sciences 35: 541-576.

Regnault, S., Pitsillides, A.A., Hutchinson, J.R. 2014. Structure, ontogeny and evolution of the patellar tendon in emus (Dromaius novaehollandiae) and other palaeognath birds. PeerJ 2:e711 http://dx.doi.org/10.7717/peerj.711

Rowe, T. 1986. Homology and evolution of the deep dorsal thigh musculature in birds and other Reptilia. Journal of Morphology 189: 327-346. 
1478 Rubenson, J., Heliams, D.B., Lloyd, D.G., \& Fournier, P.A. 2004. Gait selection in the ostrich: mechanical and metabolic characteristics of walking and running with and without an aerial phase. Proceedings of the Royal Society B: Biological Sciences 271:1091-1099.

Rubenson, J., Henry, H. T., Dimoulas, P. M., \& Marsh, R. L. 2006. The cost of running uphill: linking organismal and muscle energy use in guinea fowl (Numida meleagris). Journal of Experimental Biology 209: 2395-2408.

Rubenson, J., Lloyd, D. G., Besier, T. F., Heliams, D. B., \& Fournier, P. A. 2007. Running in ostriches (Struthio camelus): three-dimensional joint axes alignment and joint kinematics. Journal of Experimental Biology 210: 2548-2562.

Rubenson, J., Lloyd, D. G., Heliams, D. B., Besier, T. F., \& Fournier, P. A. 2011. Adaptations for economical bipedal running: the effect of limb structure on three-dimensional joint mechanics. Journal of the Royal Society Interface 8: 740-755.

Russell, D. A. 1972. Ostrich dinosaurs from the Late Cretaceous of Western Canada. Canadian Journal of Earth Sciences 9:375-402.

Schaller, N. U., Herkner, B., Villa, R., \& Aerts, P. 2009. The intertarsal joint of the ostrich (Struthio camelus): anatomical examination and function of passive structures in locomotion. Journal of Anatomy 214: 830-847.

Schaller, N. U., D'Août, K., Villa, R., Herkner, B., \& Aerts, P. 2011. Toe function and dynamic pressure distribution in ostrich locomotion. Journal of Experimental Biology 214: 1123-1130.

Sellers, W. I., Margetts, L., Coria, R. A., \& Manning, P. L. (2013). March of the titans: the locomotor capabilities of sauropod dinosaurs. PloS one 8(10): e78733.

Smith, N. C., Wilson, A. M., Jespers, K. J., \& Payne, R. C. 2006. Muscle architecture and functional anatomy of the pelvic limb of the ostrich (Struthio camelus). Journal of Anatomy, 209: 765-779.

Smith, N. C., Payne, R. C., Jespers, K. J., \& Wilson, A. M. 2007. Muscle moment arms of pelvic limb muscles of the ostrich (Struthio camelus). Journal of Anatomy 211:313-324.

Smith, N. C., Jespers, K. J., \& Wilson, A. M. 2010. Ontogenetic scaling of locomotor kinetics and kinematics of the ostrich (Struthio camelus). Journal of Experimental Biology 213: 13471355 .

Smith, N. C., \& Wilson, A. M. 2013. Mechanical and energetic scaling relationships of running gait through ontogeny in the ostrich (Struthio camelus). Journal of Experimental Biology 216: 841-849. 
1522 Suzuki, T., Hosseini, A., Li, J. S., Gill IV, T. J., \& Li, G. 2012. In vivo patellar tracking and 1523 patellofemoral cartilage contacts during dynamic stair ascending. Journal of Biomechanics 45:

1524 2432-2437.

1525

1526

1527

1528

1529

1530

1531

1532

1533

1534

1535

1536

1537

1538

1539

1540

1541

1542

1543

1544

1545

1546

1547

1548

1549

1550

1551

1552

1553

1554

1555

1556

1557

1558

1559

1560

1561 Zinoviev, A. V. 2006. Notes on the hind limb myology of the Ostrich (Struthio camelus).

1562 Ornithologia 33: 53-62.

Troy, K. L., Brown, T. D., \& Conzemius, M. G. 2009. Contact stress distributions on the femoral head of the emu (Dromaius novaehollandiae). Journal of Biomechanics 42: 2495-2500.

Tsaopoulos, D. E., Baltzopoulos, V., Richards, P. J., \& Maganaris, C. N. 2007. In vivo changes in the human patellar tendon moment arm length with different modes and intensities of muscle contraction. Journal of Biomechanics 40: 3325-3332.

Vanden Berge, J.C. 1982. Notes on the myology of the pelvic limb in kiwi (Apteryx) and in other birds. The Auk 99:309-315.

Velotto, S., \& Crasto, A. 2004. Histochemical and morphometrical characterization and distribution of fibre types in four muscles of ostrich (Struthio camelus). Anatomia Histologia Embryologia 33:251-256.

Walker, P. S., Rovick, J. S., \& Robertson, D. D. 1988. The effects of knee brace hinge design and placement on joint mechanics. Journal of Biomechanics 21:965-974.

Watson, R. R., Rubenson, J., Coder, L., Hoyt, D. F., Propert, M. W., \& Marsh, R. L. 2011. Gaitspecific energetics contributes to economical walking and running in emus and ostriches.

Proceedings of the Royal Society B: Biological Sciences 278: 2040-2046.

Weissengruber, G.E., Forstenpointner, G., \& Gangl, D. 2003. Gut zu Fuß - funktionellanatomische Aspekte des bipeden Laufens deim Afrikanischen Strauß (Struthio camelus Linné, 1758). Veterinary Medicine Austria Wiener Tierärztliche Monatsschrift 90:67-78.

Young, R. P., Scott, S. H., \& Loeb, G. E. 1993. The distal hindlimb musculature of the cat: multiaxis moment arms at the ankle joint. Experimental Brain Research 96: 141-151.

Zajac, F. E. 1989. Muscle and tendon: properties, models, scaling, and application to biomechanics and motor control. Critical Reviews in Biomedical Engineering 17:359-411.

Zarucco, L., Wisner, E. R., Swanstrom, M. D., \& Stover, S. M. 2006. Image fusion of computed tomographic and magnetic resonance images for the development of a three-dimensional musculoskeletal model of the equine forelimb. Veterinary Radiology \& Ultrasound 47: 553-562. 


\section{Table $\mathbf{1}$ (on next page)}

Table 1

Joint axes for the ostrich musculoskeletal model. Each joint centre is listed in $(x, y, z)$ coordinate space as a distance from the segment origin. The pes was $0.141 \mathrm{~m}$ long and an interphalangeal joint's location is noted here in the final row, but was not included in the model. Each joint was defined relative to the one proximal to it, with the pelvis segment placed at the origin of the world coordinate system. 
2

\begin{tabular}{lrrrll} 
Joint or segment & $\begin{array}{r}\text { centre } \\
\mathrm{x}(\mathrm{m})\end{array}$ & $\begin{array}{r}\text { centre } \\
\mathrm{y}(\mathrm{m})\end{array}$ & $\begin{array}{r}\text { centre } \\
\mathrm{z}(\mathrm{m})\end{array}$ & $\begin{array}{l}\text { Motion } \\
\text { axes }\end{array}$ & Ranges of motion $\left(^{\circ}\right)$ \\
\hline Pelvis & 0 & 0 & 0 & $\mathrm{x}, \mathrm{y}, \mathrm{z}$ & {$[-180 / 180 ;-180 / 180 ;-180 / 180]$} \\
Hip (acetabular/antitrochanteric) & 0 & 0 & 0.0355 & $\mathrm{x}, \mathrm{y}, \mathrm{z}$ & {$[-45 / 45 ;-45 / 45 ;-65 / 10]$} \\
Knee (femorotibial) & 0 & -0.2338 & 0.0543 & $\mathrm{x}, \mathrm{y}, \mathrm{z}$ & {$[-45 / 45 ;-45 / 45 ;-180 / 10]$} \\
Ankle (intertarsal) & 0 & -0.442 & 0 & $\mathrm{x}, \mathrm{z}$ & {$[-45 / 45 ;-10 ;-10 / 180]$} \\
Metatarsophalangeal (MTP III) & 0 & -0.426 & 0 & $\mathrm{z}$ & {$[5 ; 24 ;-180 / 90]$} \\
[Proximal interphalangeal (D III)] & {$[0$} & -0.089 & $0]$ & {$[\mathrm{z}]$} & {$[$ not estimated] }
\end{tabular}

\section{3}




\section{Table 2 (on next page)}

Table 2

Muscles included in the ostrich musculoskeletal model (ordered as anatomical/functional groups as per prior studies), with their associated abbreviations and physiological/architectural parameters. Data were obtained via dissection. Blank cells for muscle masses (ILp, ILFBp, ITCp, TCt) indicate that the second part of the muscle shares the mass value, which was divided equally to calculate $A_{\text {phys }}$ and hence $F_{\text {max }}$. 


\begin{tabular}{|c|c|c|c|c|c|}
\hline $\begin{array}{l}\text { Muscle } \\
\text { abbreviation }\end{array}$ & Muscle full name & $\begin{array}{l}\text { Muscle } \\
\text { mass; } \\
\mathrm{m}_{\text {musc }} \\
(\mathrm{kg})\end{array}$ & $\begin{array}{l}\text { Fascicle } \\
\text { length; } \\
\text { L (m) }\end{array}$ & $\begin{array}{l}\text { Pennation } \\
\text { angle; } \theta \\
\left({ }^{\circ}\right)\end{array}$ & $\begin{array}{l}\text { Maximal } \\
\text { isometric } \\
\text { force; } \\
\mathrm{F}_{\max }(\mathrm{N})\end{array}$ \\
\hline $\mathrm{IC}$ & M. iliotibialis cranialis & 0.3788 & 0.174 & 0 & 615 \\
\hline ILa & M. iliotibialis lateralis (cranial part) & 1.074 & 0.174 & 0 & 875 \\
\hline ILp & M. iliotibialis lateralis (caudal part) & & 0.174 & 0 & 875 \\
\hline AMB1 & M. ambiens, ventral (pubic) head & 0.093 & 0.039 & 10 & 672 \\
\hline AMB2 & M. ambiens, dorsal (iliac) head & 0.1994 & 0.044 & 15 & 1240 \\
\hline FMTL & M. femorotibialis lateralis & 0.3181 & 0.088 & 15 & 992 \\
\hline FMTIM & M. femorotibialis intermedius & 0.387 & 0.084 & 25 & 1180 \\
\hline FMTM & M. femorotibialis medialis & 0.272 & 0.089 & 30 & 753 \\
\hline ILFBa & M. iliofibularis (cranial part) & 1.0623 & 0.176 & 0 & 867 \\
\hline ILFBp & M. iliofibularis (caudal part) & & 0.176 & 0 & 867 \\
\hline ITCa & M. iliotrochantericus caudalis (cranial part) & 0.3114 & 0.064 & 25 & 622 \\
\hline ITCp & M. iliotrochantericus caudalis (caudal part) & & 0.064 & 25 & 622 \\
\hline IFE & M. iliofemoralis externus & 0.03264 & 0.025 & 25 & 331 \\
\hline ITM & M. iliotrochantericus medius & 0.0256 & 0.058 & 0 & 125 \\
\hline ITCR & M. iliotrochantericus cranialis & 0.0432 & 0.053 & 10 & 228 \\
\hline IFI & M. iliofemoralis internus & 0.0407 & 0.041 & 0 & 284 \\
\hline FCM & M. flexor cruris medialis & 0.1192 & 0.036 & 35 & 767 \\
\hline FCLP & M. flexor cruris lateralis pars pelvica & 0.3182 & 0.24 & 0 & 376 \\
\hline FCLA & M. flexor cruris lateralis pars accessoria & 0.0211 & 0.125 & 0 & 47.8 \\
\hline ISF & M. ischiofemoralis & 0.0348 & 0.033 & 15 & 290 \\
\hline PIFML & Mm. puboischiofemorales medialis + lateralis & 0.1273 & 0.089 & 15 & 389 \\
\hline $\mathrm{OM}$ & M. obturatorius medialis & 0.457 & 0.055 & 25 & 2160 \\
\hline CFP & M. caudofemoralis pars pelvica (et caudalis) & 0.3069 & 0.108 & 15 & 778 \\
\hline GL & M. gastrocnemius pars lateralis & 0.5706 & 0.12 & 20 & 1269 \\
\hline GIM & M. gastrocnemius pars intermedia & 0.2526 & 0.125 & 15 & 552 \\
\hline GM & M. gastrocnemius pars medialis & 0.762 & 0.094 & 20 & 2160 \\
\hline FL & M. fibularis longus & 0.4791 & 0.081 & 20 & 1570 \\
\hline FDL & M. flexor digitorum longus & 0.1424 & 0.048 & 20 & 782 \\
\hline FPPD3 & M. flexor perforans et perforatus digitorum 3 & 0.0822 & 0.025 & 30 & 798 \\
\hline FPD3 & M. flexor perforatus digitorum 3 & 0.1605 & 0.017 & 35 & 2220 \\
\hline FPD4 & M. flexor perforatus digitorum 4 & 0.0955 & 0.026 & 20 & 992 \\
\hline FHL & M. flexor hallucis longus & 0.0505 & 0.04 & 25 & 324 \\
\hline EDL & M. extensor digitorum longus & 0.115 & 0.049 & 30 & 576 \\
\hline TCf & M. tibialis cranialis (femoral head) & 0.165 & 0.045 & 25 & 474 \\
\hline $\mathrm{TCt}$ & M. tibialis cranialis (tibial head) & & 0.045 & 25 & 474 \\
\hline
\end{tabular}




\section{Table 3(on next page)}

Table 3

Muscle wrapping surfaces assumed in the ostrich musculoskeletal model, with dimensions.

Examples are in Figure 5. " $* "$ " the torus's outer and inner diameters, not radius and length, respectively. 


\begin{tabular}{|c|c|c|c|c|c|c|c|c|c|c|c|}
\hline Muscle(s) & Location & Shape & $\mathrm{r}(\mathrm{x})$ & $\mathrm{r}(\mathrm{y})$ & $\mathrm{r}(\mathrm{z})$ & $t(x)$ & $\mathrm{t}(\mathrm{y})$ & $\mathrm{t}(\mathrm{z})$ & Radius & Length & \\
\hline ILFB & pelvis & cylinder & 17.11 & 57.87 & -34.76 & 0.0309 & -0.0609 & 0.0622 & 0.095 & 1.000 & \\
\hline PIFML & pelvis & cylinder & 0.92 & -5.72 & -29.06 & 0.0020 & 0.0820 & 0.1000 & 0.170 & 0.500 & \\
\hline CFP & pelvis & cylinder & -8.51 & 0.41 & 35.68 & -0.0211 & 0.0722 & 0.1396 & 0.090 & 0.500 & \\
\hline ITC,ITM & femur & cylinder & -16.00 & 19.60 & 0.00 & 0.0086 & -0.0017 & -0.0067 & 0.020 & 0.500 & \\
\hline ITCR & femur & cylinder & -16.00 & -2.17 & -0.19 & 0.0395 & -0.0379 & -0.0034 & 0.020 & 0.500 & \\
\hline ITCR & femur & cylinder & 74.00 & 0.00 & -19.60 & 0.0086 & -0.0882 & 0.0181 & 0.055 & 0.500 & \\
\hline GL,GIM,FHL,FPD3 & femur & cylinder & 37.16 & 40.49 & -20.39 & -0.0197 & -0.2006 & 0.0799 & 0.015 & 0.200 & \\
\hline ILFBp & femur & torus & 51.43 & -21.08 & -22.24 & -0.0202 & -0.1827 & 0.0609 & $0.01^{*}$ & $0.08^{*}$ & \\
\hline FMTIM,FMTL & tibiotarsus & cylinder & 0.00 & 0.00 & 44.69 & -0.0014 & 0.0103 & 0.0093 & 0.038 & 0.500 & \\
\hline GL,GIM,GM & tibiotarsus & cylinder & 0.00 & 0.00 & 0.00 & -0.0058 & -0.4435 & -0.0090 & 0.040 & 0.150 & \\
\hline FP\&PD3,FPD3,FPD4 & tibiotarsus & cylinder & 6.47 & -7.64 & 40.43 & 0.0031 & -0.4537 & 0.0090 & 0.030 & 0.200 & \\
\hline FDL,FHL & tibiotarsus & cylinder & 0.00 & 0.00 & 40.00 & -0.0014 & -0.4501 & 0.0001 & 0.030 & 0.500 & \\
\hline AMB2 & tibiotarsus & cylinder & 0.00 & 0.00 & 0.00 & 0.0250 & 0.0126 & -0.0012 & 0.038 & 0.100 & \\
\hline IC & tibiotarsus & cylinder & 0.00 & 5.00 & 0.00 & -0.0143 & 0.0098 & -0.0054 & 0.030 & 0.500 & \\
\hline EDL,TCf,TCt & tibiotarsus & cylinder & 3.37 & -15.47 & 0.32 & 0.0111 & -0.4530 & 0.0009 & 0.020 & 0.100 & \\
\hline FP\&PD3,FPD3,FL & tarsometatarsus & cylinder & 0.64 & -6.17 & -1.51 & -0.0020 & -0.4296 & 0.0002 & 0.023 & 0.100 & \\
\hline FDL,FHL & tarsometatarsus & cylinder & -3.48 & -13.61 & -0.29 & 0.0025 & -0.4319 & -0.0024 & 0.022 & 0.100 & \\
\hline FPD4 & tarsometatarsus & cylinder & 0.00 & -35.00 & 0.00 & 0.0002 & -0.4326 & 0.0025 & 0.017 & 0.100 & \\
\hline EDL & tarsometatarsus & cylinder & -1.19 & -2.69 & 0.68 & 0.0029 & -0.4312 & -0.0152 & 0.023 & 0.100 & \\
\hline Muscle & Location & $\underline{\text { Shape }}$ & $\underline{\mathrm{r}(\mathrm{x})}$ & $\underline{\mathrm{r}(\mathrm{y})}$ & $\underline{\mathrm{r}(\mathrm{z})}$ & $\underline{t(x)}$ & $\underline{\mathrm{t}(\mathrm{y})}$ & $\underline{\mathrm{t}(\mathrm{z})}$ & $\frac{\text { Radius }}{\underline{(\mathrm{x})}}$ & $\frac{\text { Radius }}{(\mathrm{y})}$ & $\frac{\text { Radius }}{\underline{(\mathrm{z})}}$ \\
\hline IL & pelvis & ellipsoid & -2.79 & -1.44 & -100.93 & -0.0020 & -0.0020 & 0.0649 & 0.100 & 0.200 & 0.050 \\
\hline IFE & pelvis & ellipsoid & 0.00 & 0.00 & 0.00 & -0.0010 & 0.0110 & 0.0302 & 0.090 & 0.060 & 0.060 \\
\hline FCLP & pelvis & ellipsoid & -17.65 & 8.20 & -84.13 & -0.2258 & -0.0290 & 0.0532 & 0.075 & 0.500 & 0.050 \\
\hline
\end{tabular}




\section{Table 4(on next page)}

Table 4

Muscle actions, following results from Figures $9-20$, to describe the major 3D potential functions of each ostrich pelvic limb muscle. Classifications: $E=$ extensor, $F=f l e x o r, M=$ medial (internal) rotator, $L=$ lateral (external) rotator, $A B=$ abductor, $A D=$ adductor, $0=$ no moment arm per se despite crossing the joint. Blank cells indicate the muscle does not cross or act about the joint. " +" signs added to classifications indicate a major potential role in these functions based upon moment arm and muscle relative size (i.e., moment generation capacity), subjectively assessed. "I" combinations (F/E; M/L; AB/AD) indicate a strong sensitivity of muscle moment arm, and hence action, to joint angle. Annotation with an asterisk indicates a potential role for intrinsic stabilization about that axis of motion (see Discussion). "()" indicates that our model's single origin for each muscle (or part thereof) did not allow such an action, but sub-parts of those muscles might have such actions if modelled in more detail. 


\begin{tabular}{|c|c|c|c|c|c|c|}
\hline Muscle & $\begin{array}{l}\text { Action } \\
\text { Hip } \\
\text { F/E } \\
\end{array}$ & $\begin{array}{l}\text { Hip } \\
\text { LAR }\end{array}$ & $\begin{array}{l}\text { Hip } \\
\mathrm{Ab} / \mathrm{Ad}\end{array}$ & $\begin{array}{l}\text { Knee } \\
\text { F/E }\end{array}$ & $\begin{array}{l}\text { Ankle } \\
\text { F/E }\end{array}$ & $\begin{array}{l}\text { MTP } \\
\text { F/E }\end{array}$ \\
\hline IC & $\mathrm{F}+{ }^{*}$ & M & $\mathrm{AD}+$ & $\mathrm{F} / \mathrm{E}$ & & \\
\hline ILa & $\mathrm{F} / \mathrm{E}$ & $\mathrm{M} / \mathrm{L}$ & $\mathrm{AB}+$ & $\mathrm{E}+$ & & \\
\hline ILp & $\mathrm{E}+$ & $\mathrm{M} / \mathrm{L}$ & $\mathrm{AB}+$ & $\mathrm{E}+$ & & \\
\hline AMB1 & $E^{*}$ & $\mathrm{~L}$ & $\mathrm{AD}$ & $\mathrm{F}$ & & \\
\hline AMB2 & $\mathrm{F}^{*}$ & $\mathrm{M} / \mathrm{L}$ & $\mathrm{AD}$ & $\mathrm{E}$ & & \\
\hline FMTL & & & & $\mathrm{E}+$ & & \\
\hline FMTIM & & & & $\mathrm{E}$ & & \\
\hline FMTM & & & & $\mathrm{F}$ & & \\
\hline ILFBa & $\mathrm{E}$ & M & $\mathrm{AB}$ & $\mathrm{F}+$ & & \\
\hline ILFBp & $\mathrm{E}+$ & M & $\mathrm{AB}$ & $\mathrm{F}+$ & & \\
\hline ITCa & $\mathrm{F} / \mathrm{E}^{*}$ & $\mathrm{M}+$ & $\mathrm{AB} / \mathrm{AD}$ & & & \\
\hline ITCp & $\mathrm{F} / \mathrm{E}^{*}$ & $\mathrm{M}+$ & $\mathrm{AB} / \mathrm{AD}$ & & & \\
\hline IFE & F & $\mathrm{M} / \mathrm{L}$ & $\mathrm{AB}$ & & & \\
\hline ITM & $\mathrm{F} / \mathrm{E}^{*}$ & M & $\mathrm{AB} / \mathrm{AD}$ & & & \\
\hline ITCR & $\mathrm{F} / \mathrm{E}^{*}$ & $\mathrm{M}+$ & $\mathrm{AB} / \mathrm{AD}$ & & & \\
\hline IFI & F & $\mathrm{M} / \mathrm{L}$ & $\mathrm{AD}$ & & & \\
\hline $\mathrm{FCM}$ & $\mathrm{E}$ & M & $\mathrm{AB}$ & F & & \\
\hline FCLP & $\mathrm{E}+$ & $\mathrm{M}+$ & $\mathrm{AB}+$ & F & & \\
\hline FCLA & $\mathrm{E}$ & M & $\mathrm{AB}$ & & & \\
\hline ISF & $\mathrm{F} / \mathrm{E}^{*}$ & $\mathrm{~L}$ & $\mathrm{AB}$ & & & \\
\hline PIFML & E & $\mathrm{L}$ & $\mathrm{AB}$ & & & \\
\hline $\mathrm{OM}$ & $\mathrm{F}+$ & $\mathrm{L}+$ & $\mathrm{AB} / \mathrm{AD}^{*}$ & & & \\
\hline CFP & $\mathrm{E}$ & $\mathrm{L}$ & $\mathrm{AB}$ & & & \\
\hline GL & & & & F & $\mathrm{E}+$ & \\
\hline GIM & & & & F & E & \\
\hline GM & & & & $(\mathrm{F} / \mathrm{E})$ & $\mathrm{E}+$ & \\
\hline $\mathrm{FL}$ & & & & $\mathrm{E}$ & $\mathrm{F}^{*}$ & $\mathrm{~F}$ \\
\hline FDL & & & & & $\mathrm{E}+$ & $\mathrm{F}+$ \\
\hline FPPD3 & & & & $(\mathrm{F} / \mathrm{E})$ & $\mathrm{E}+$ & $\mathrm{F}+$ \\
\hline FPD3 & & & & $(\mathrm{F} / \mathrm{E})$ & $\mathrm{E}+$ & $\mathrm{F}+$ \\
\hline FPD4 & & & & (F) & $\mathrm{E}+$ & $\mathrm{F}+$ \\
\hline FHL & & & & (F) & E & $\mathrm{F}$ \\
\hline EDL & & & & & $\mathrm{F}^{*}{ }^{*}$ & $\mathrm{E}+$ \\
\hline TCf & & & & 0 & $\mathrm{~F}^{*}{ }^{*}$ & $\mathrm{E}+$ \\
\hline $\mathrm{TCt}$ & & & & & $\mathrm{F}^{*}{ }^{*}$ & $\mathrm{E}+$ \\
\hline
\end{tabular}


1

Figure 1

Digitizing apparatus used during anatomical dissection of ostrich. "LED Ref" indicates the proximal (in trochanteric crest of the femur) and distal (in tibiotarsus by the ankle) reference frames, "Dig. Probe" indicates the digitizing probe used to collect landmarks.

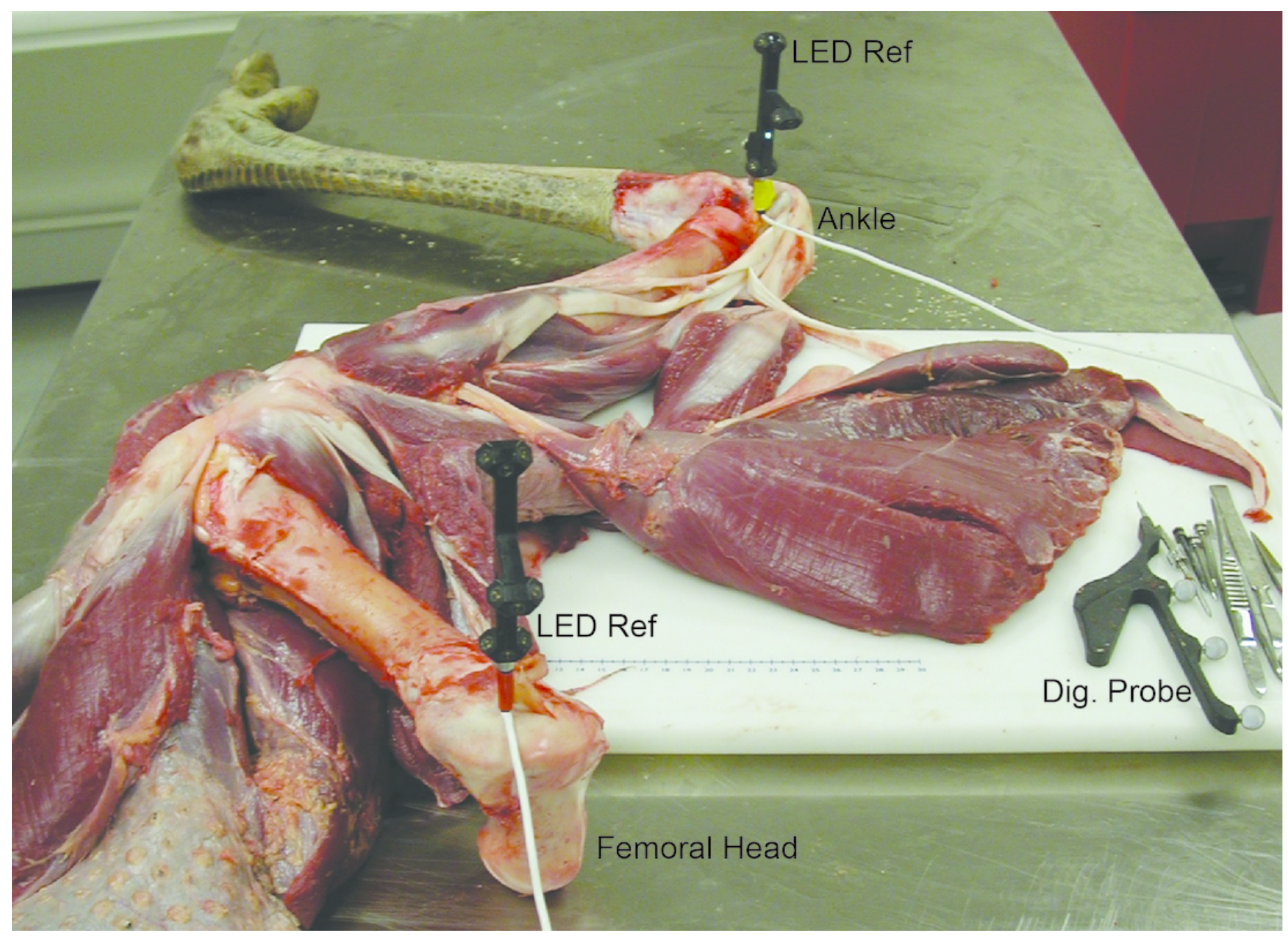


2

Figure 2

Ostrich model joint axes $(x, y, z)$ shown in right lateral $(A)$ and oblique right dorsolateral $(B)$ views. The $x$-axis corresponded to ab/adduction, the $y$-axis to long-axis rotation, and the zaxis to flexion/extension.
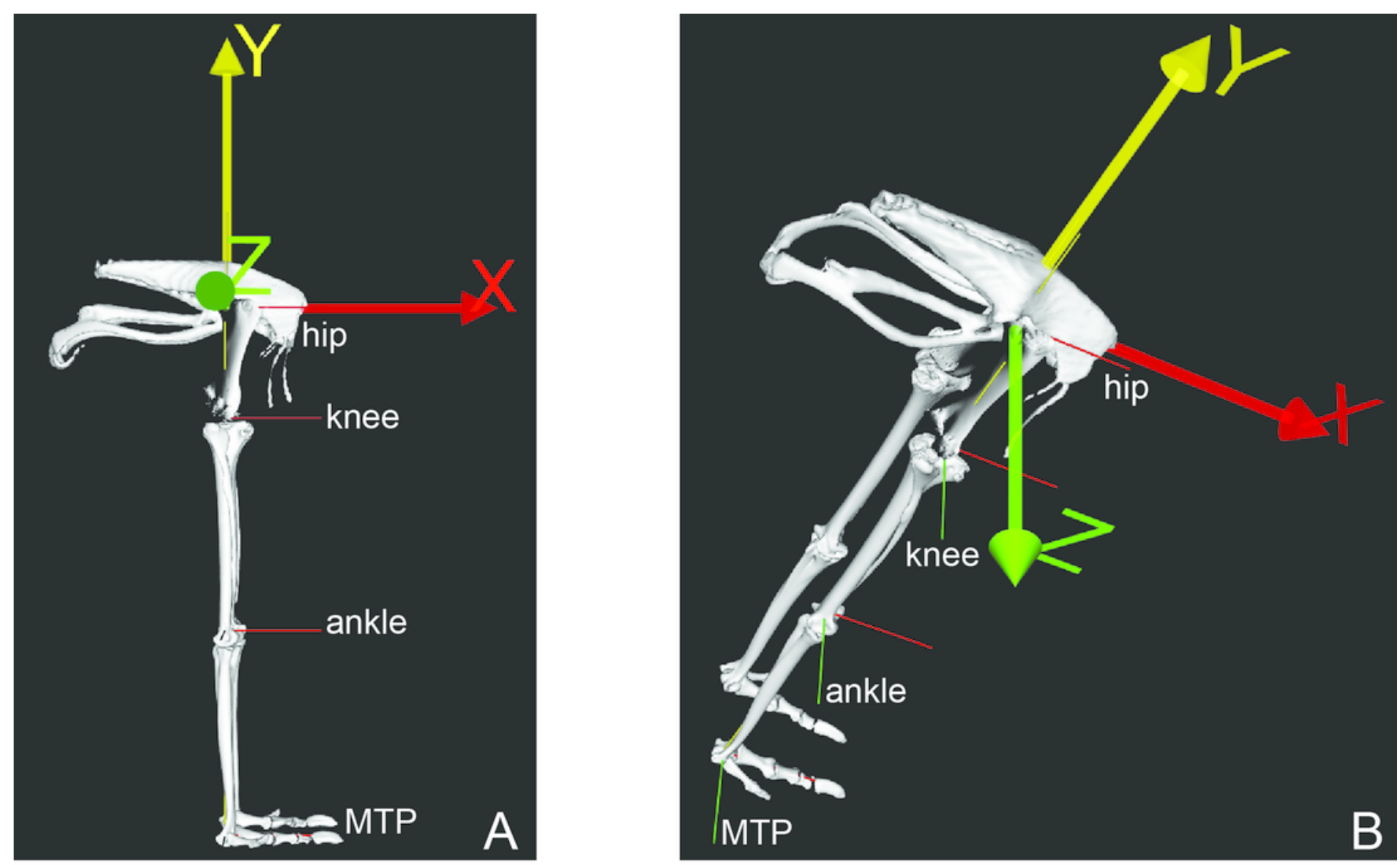
3

Figure 3

Ostrich musculoskeletal model in right lateral view, with muscle-tendon units labelled (red lines). See Table 2 for muscle abbreviations. 


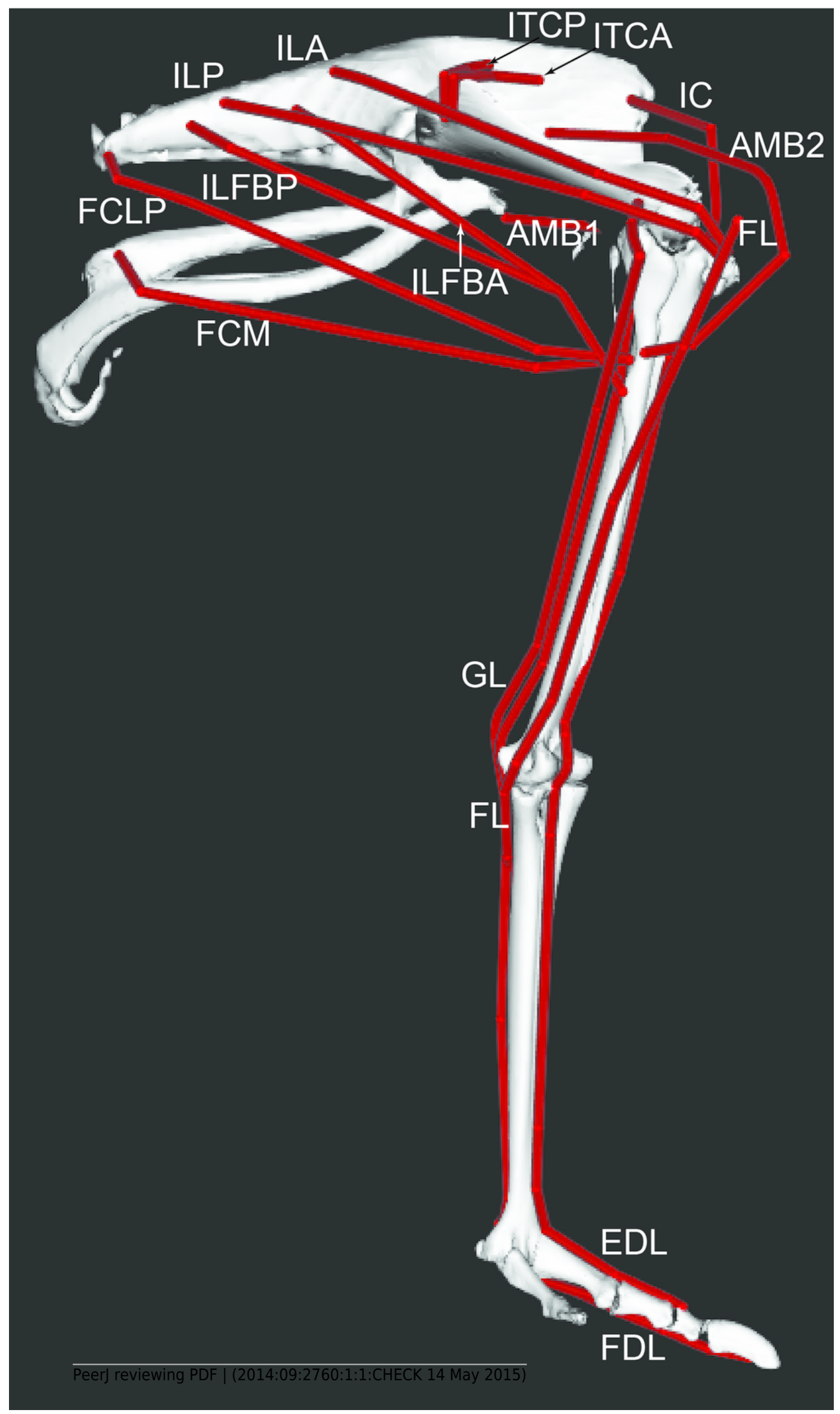


4

Figure 4

Ostrich musculoskeletal model in right caudolateral view, with muscle-tendon units labelled (red lines). See Table 2 for muscle abbreviations.

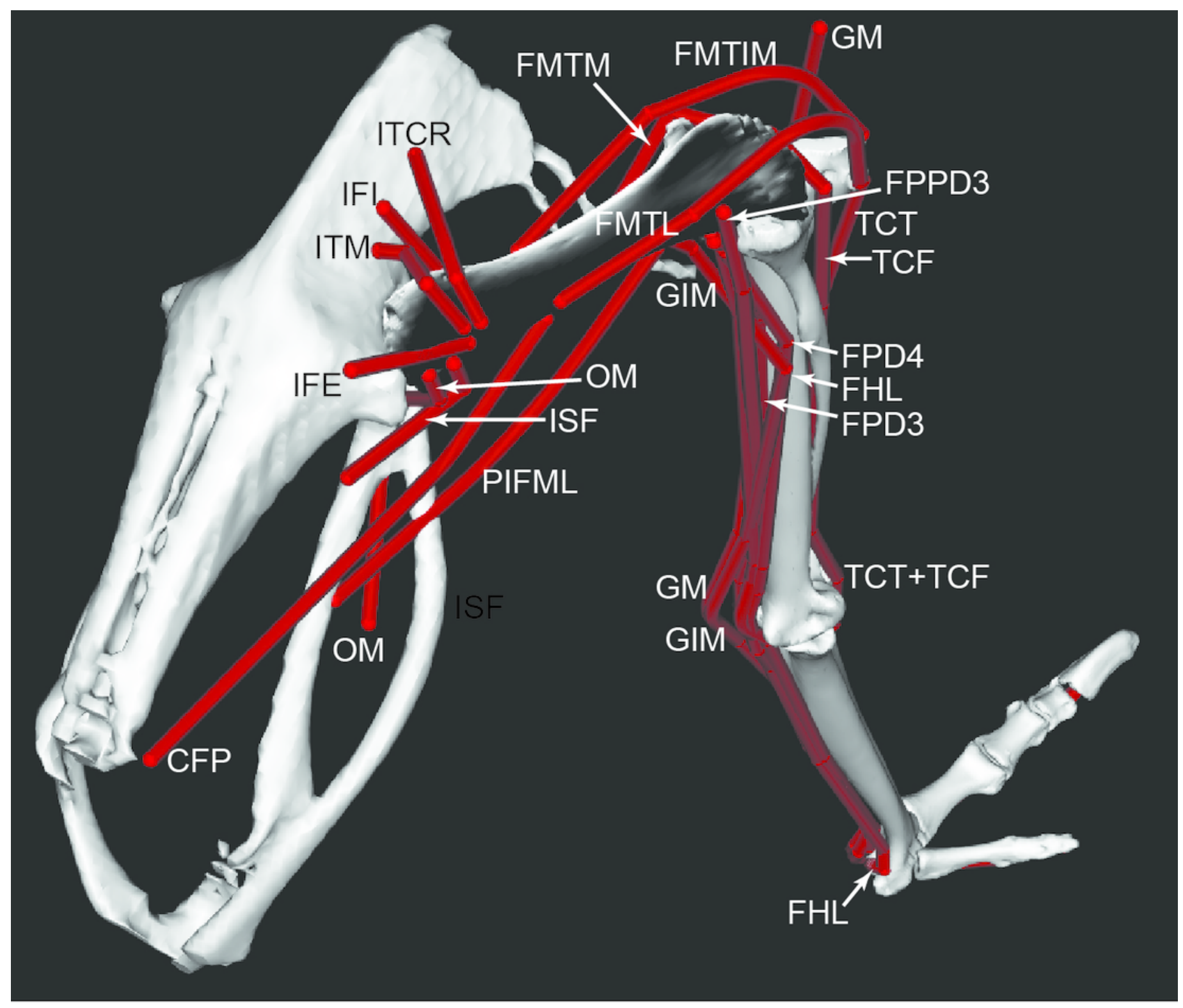




\section{5}

Figure 5

Ostrich musculoskeletal model: wrapping surface examples. See Table 2 for muscle abbreviations. Lateral (A), craniolateral (B), and caudolateral (C) views of eight muscle wrapping objects (in blue), as half and whole cylinders, ellipses and a torus. The PIFML and ILFB wrapping surfaces are shown as meshes, for added clarity. 

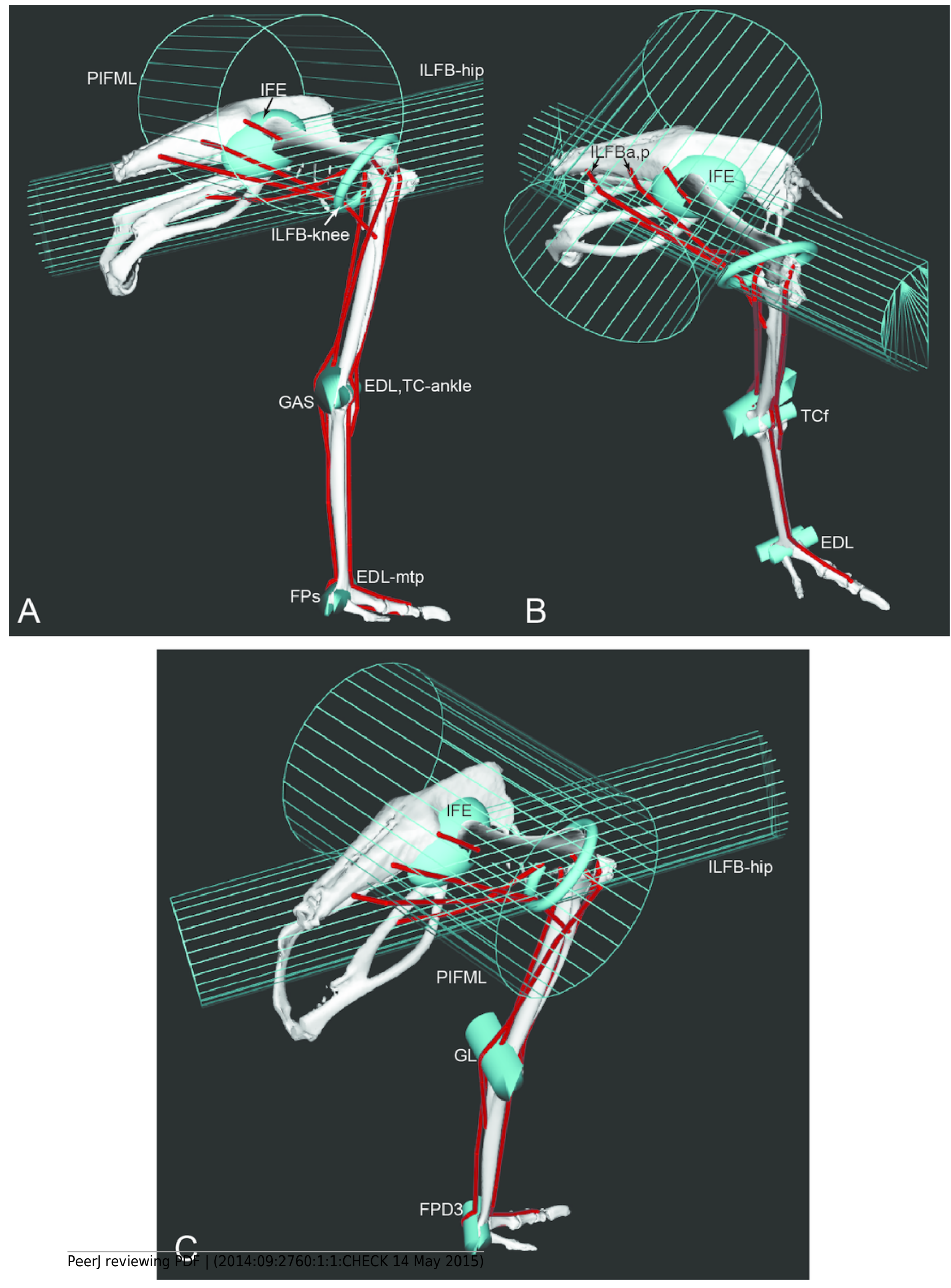


\section{6}

Figure 6

Maximal muscle moments about proximal limb joints (hip and knee), for representative walking and running trials (see Methods). "F-L" curves incorporate effects of muscle forcelength properties into moment calculations; "Fmax" curves only assume maximal isometric muscle stress and thus ignore F-L effects. The shaded area represents the stance phase, and the vertical dashed line is mid-stance (i.e., 50\%).
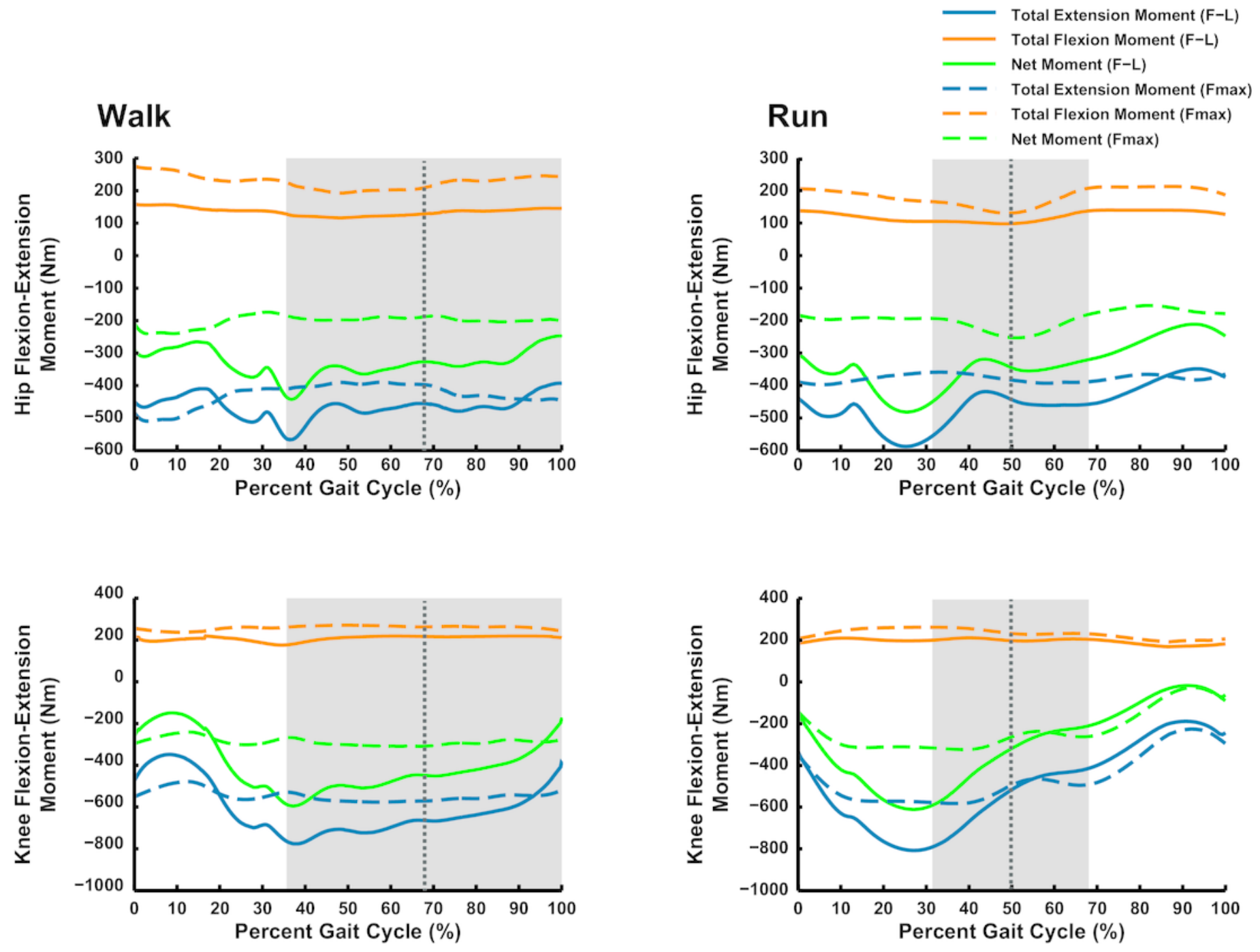


\section{7}

\section{Figure 7}

Maximal muscle moments about distal limb joints (ankle and metatarsophalangeal [MTP]), for representative walking and running trials (see Methods). See caption for Figure 6.
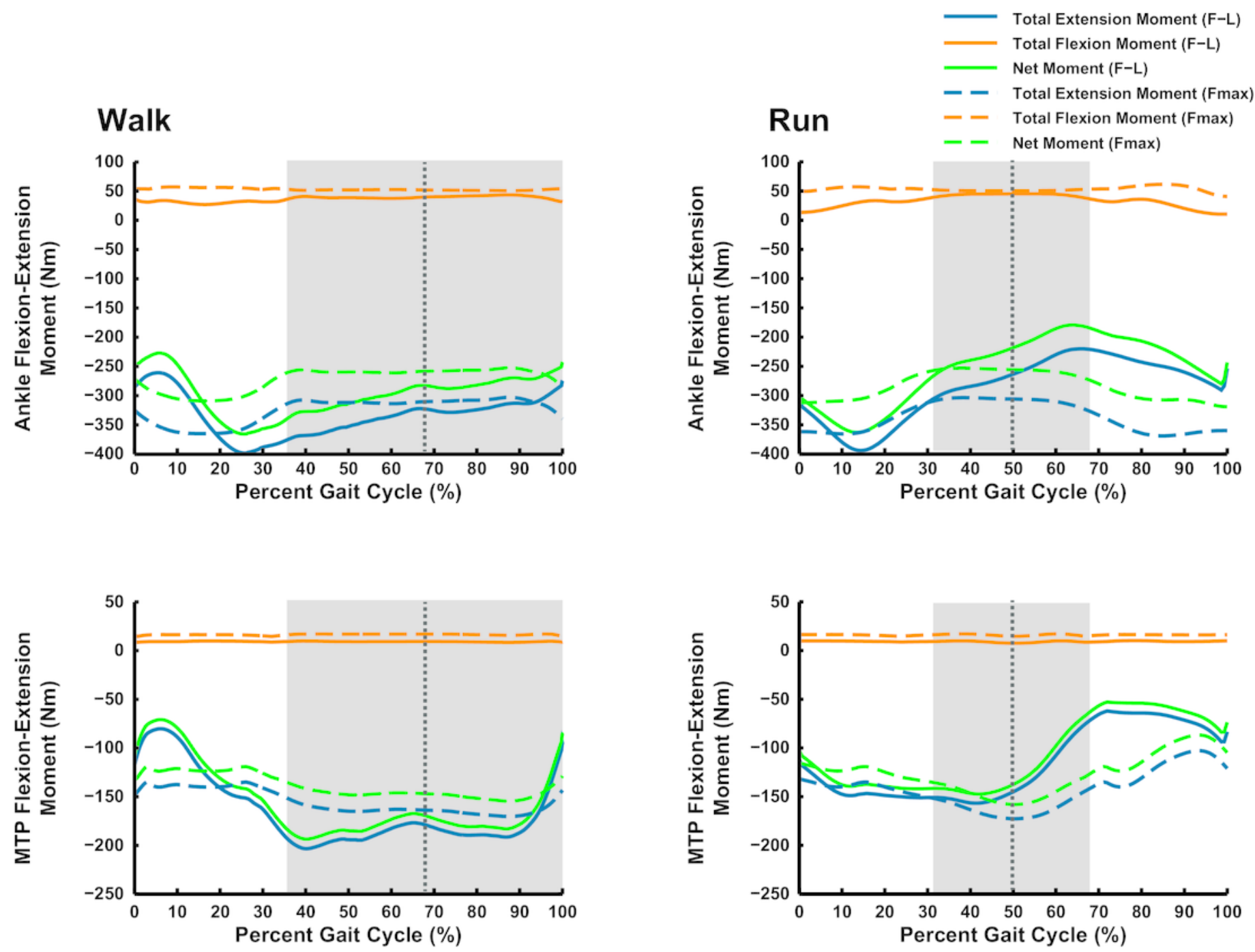
8

Figure 8

Sum of extensor moment arms (left column) or flexor moment arms (right column) normalized by sum of maximal extensor or flexor moment arms, plotted against extension or flexion joint angle for the hip, knee and ankle joints (MTP joint data follow Figure 20), with representative mid-stance limb poses for walking and running indicated. 

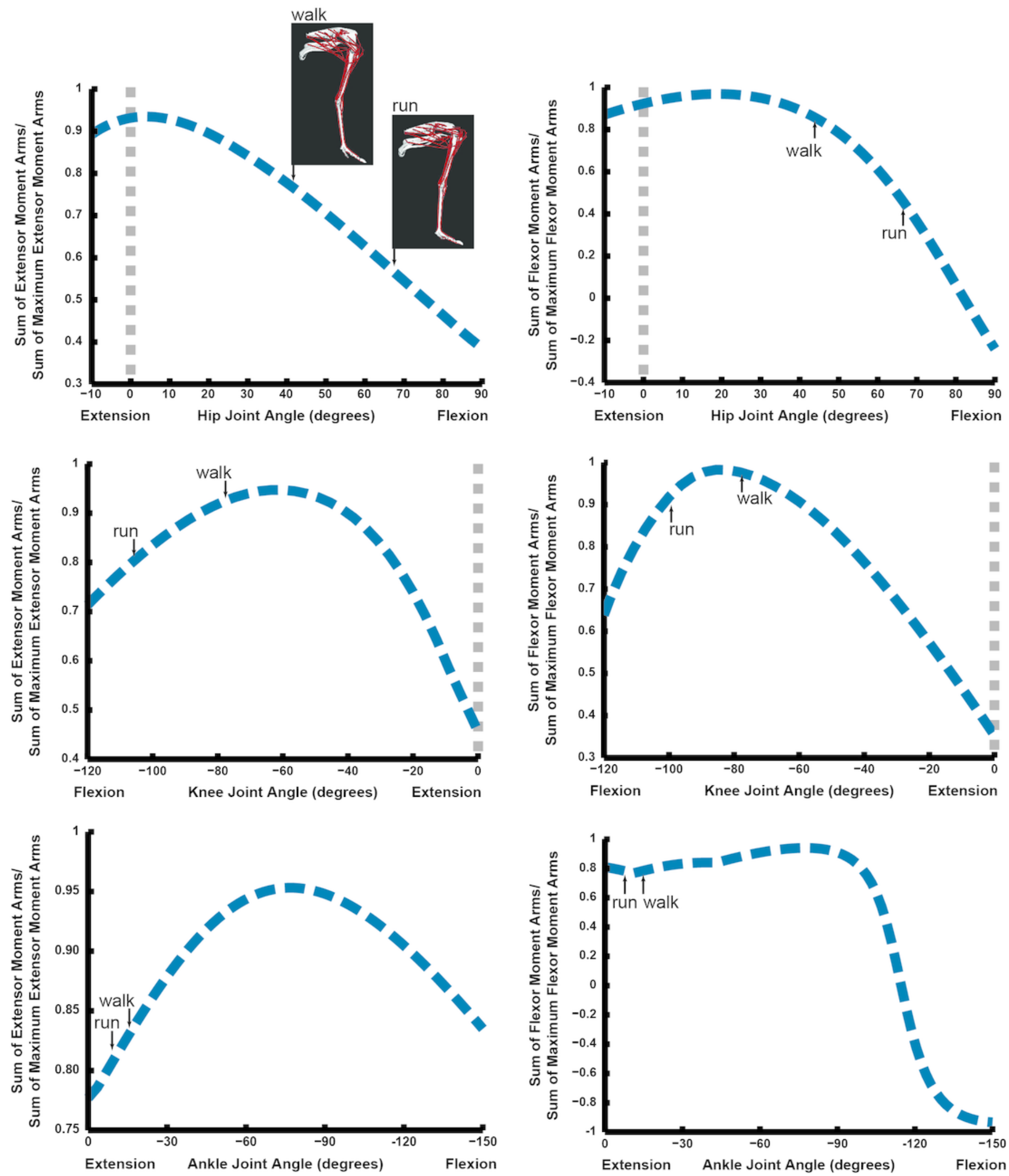


\section{9}

Figure 9

Hip flexor/extensor moment arms plotted against joint angle for key proximal thigh muscles in our model, with corresponding data from Smith et al. (2005) labelled as "Smith" and from Bates and Schachner (2012) labelled as "Bates". Extreme extended/flexed right hip joint poses shown along the $x$-axis. Muscle abbreviations are in Table 2. Colours and line solidity are kept as consistent as feasible to reflect the study (e.g. Smith in blue solid lines) and muscle (e.g. reddish shades for parts of the AMB muscle in our data).

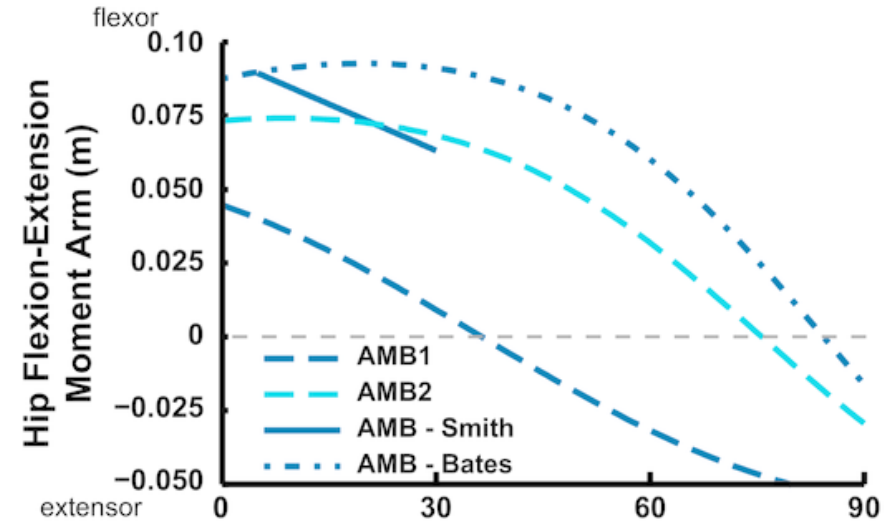

Hip Joint Angle (degrees)

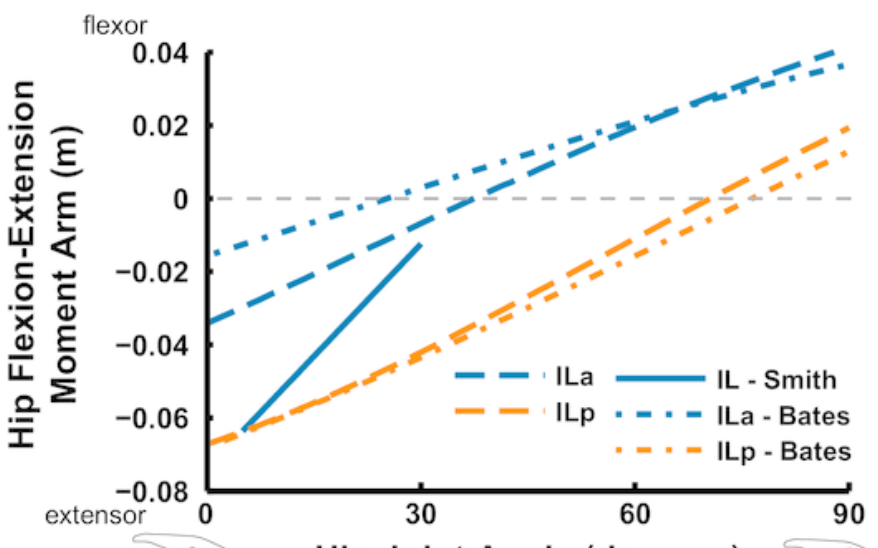

Hip Joint Angle (degrees)

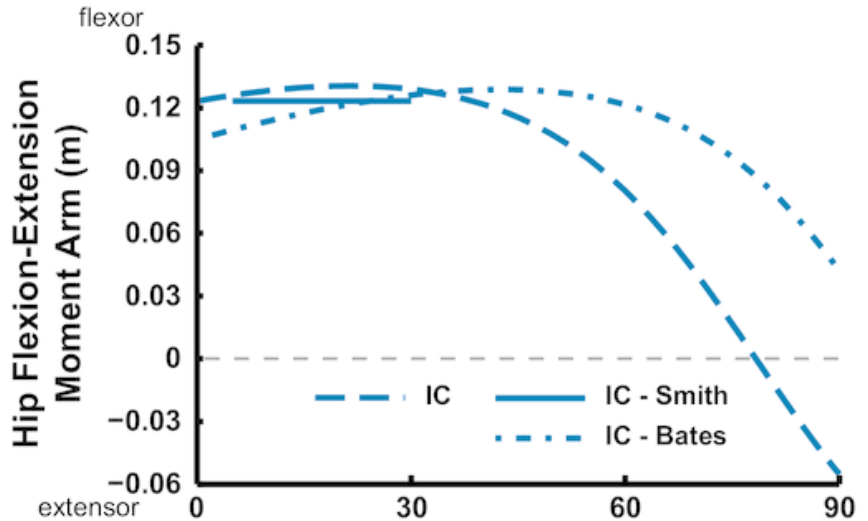

Hip Joint Angle (degrees)

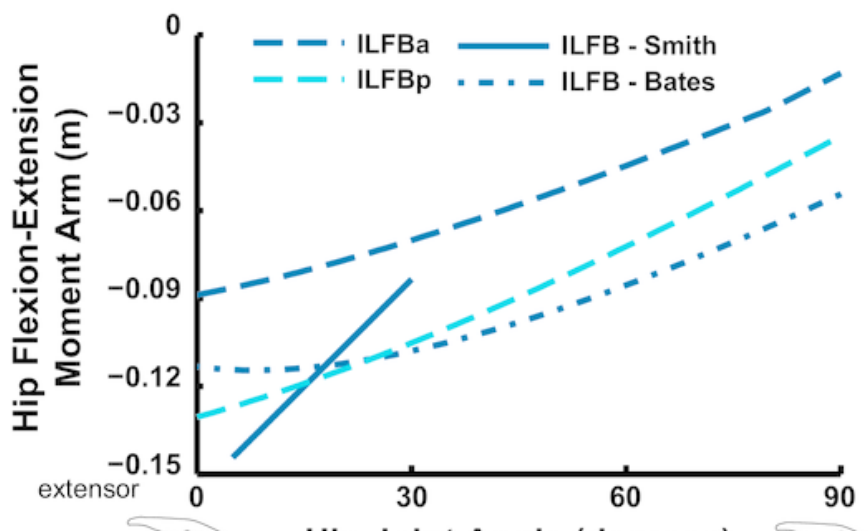

Hip Joint Angle (degrees) 
Figure 10

Hip flexor/extensor moment arms plotted against joint angle for key proximal thigh muscles. See caption for Figure 9. Dot-dashed lines represent "Bates" data here, whereas our data are in dashed lines.
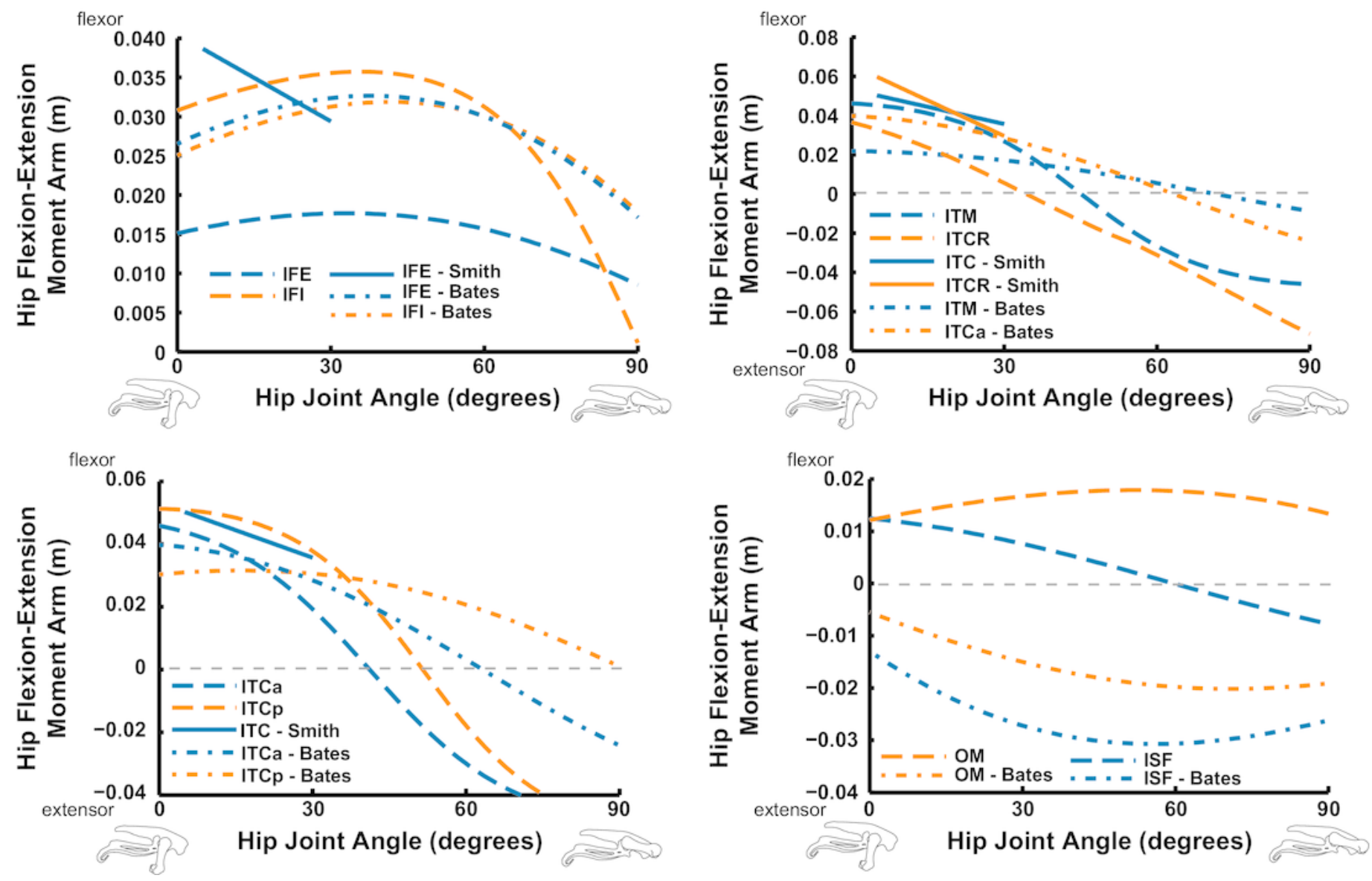
11

Figure 11

Hip flexor/extensor moment arms plotted against joint angle for key proximal thigh muscles.

See captions for Figures 9 and 10.
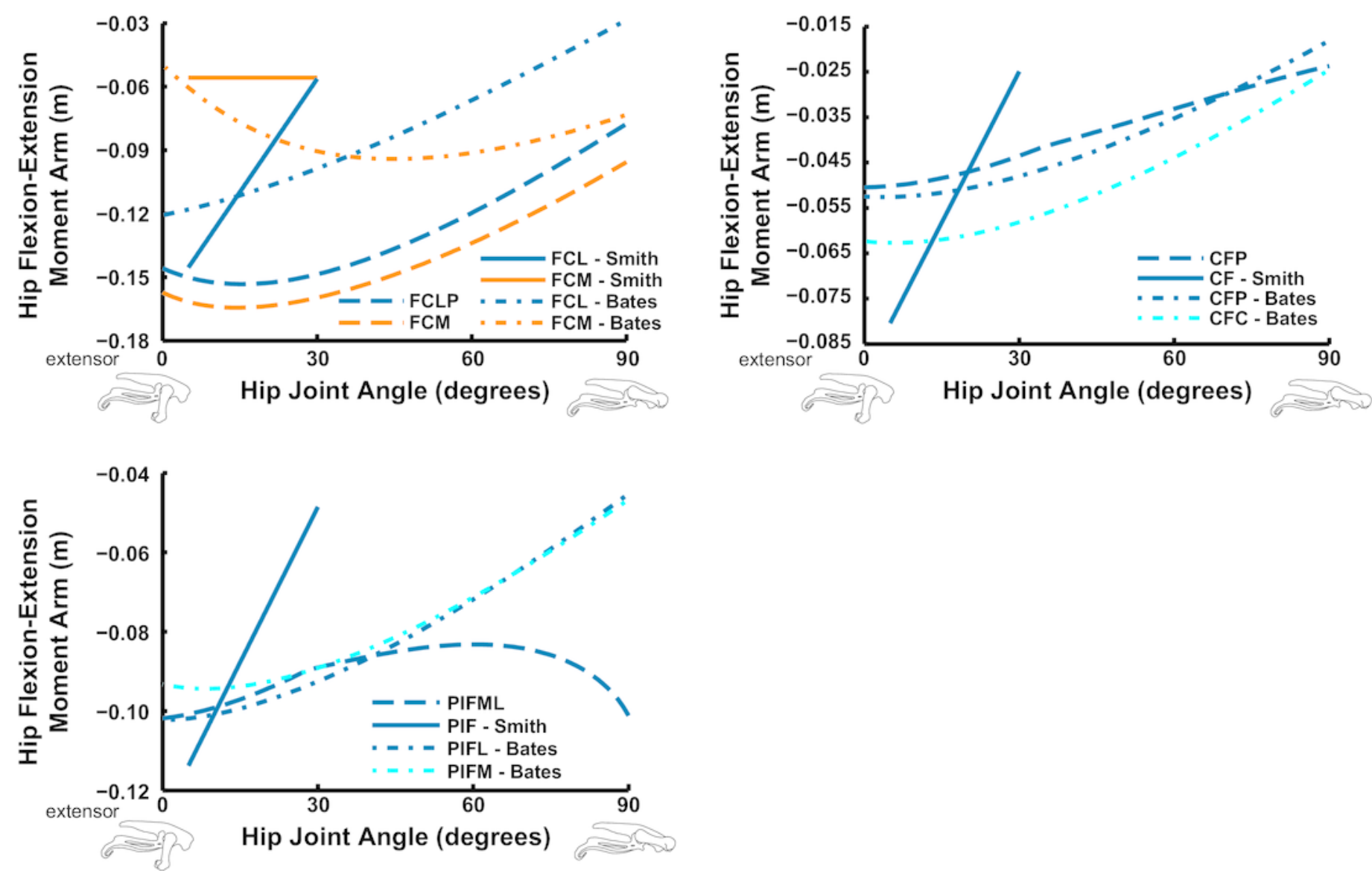
Figure 12

Hip long-axis rotation (LAR) moment arms plotted against hip flexion/extension joint angle for key proximal thigh muscles. See caption for Figure 9.
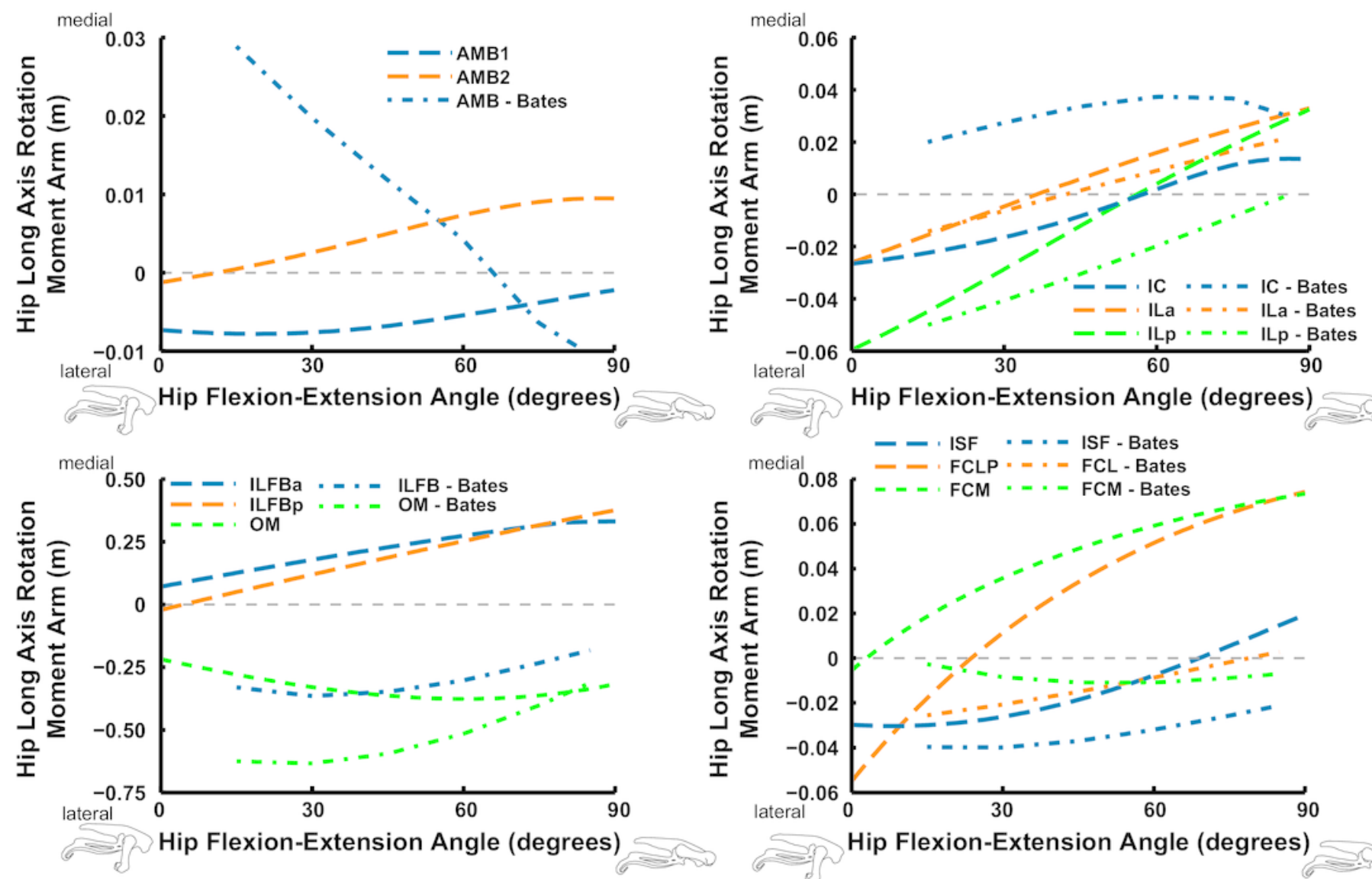

Hip Flexion-Extension Angle (degrees) 


\section{3}

Figure 13

Hip long-axis rotation (LAR) moment arms plotted against hip flexion/extension joint angle for key proximal thigh muscles. See caption for Figure 9.
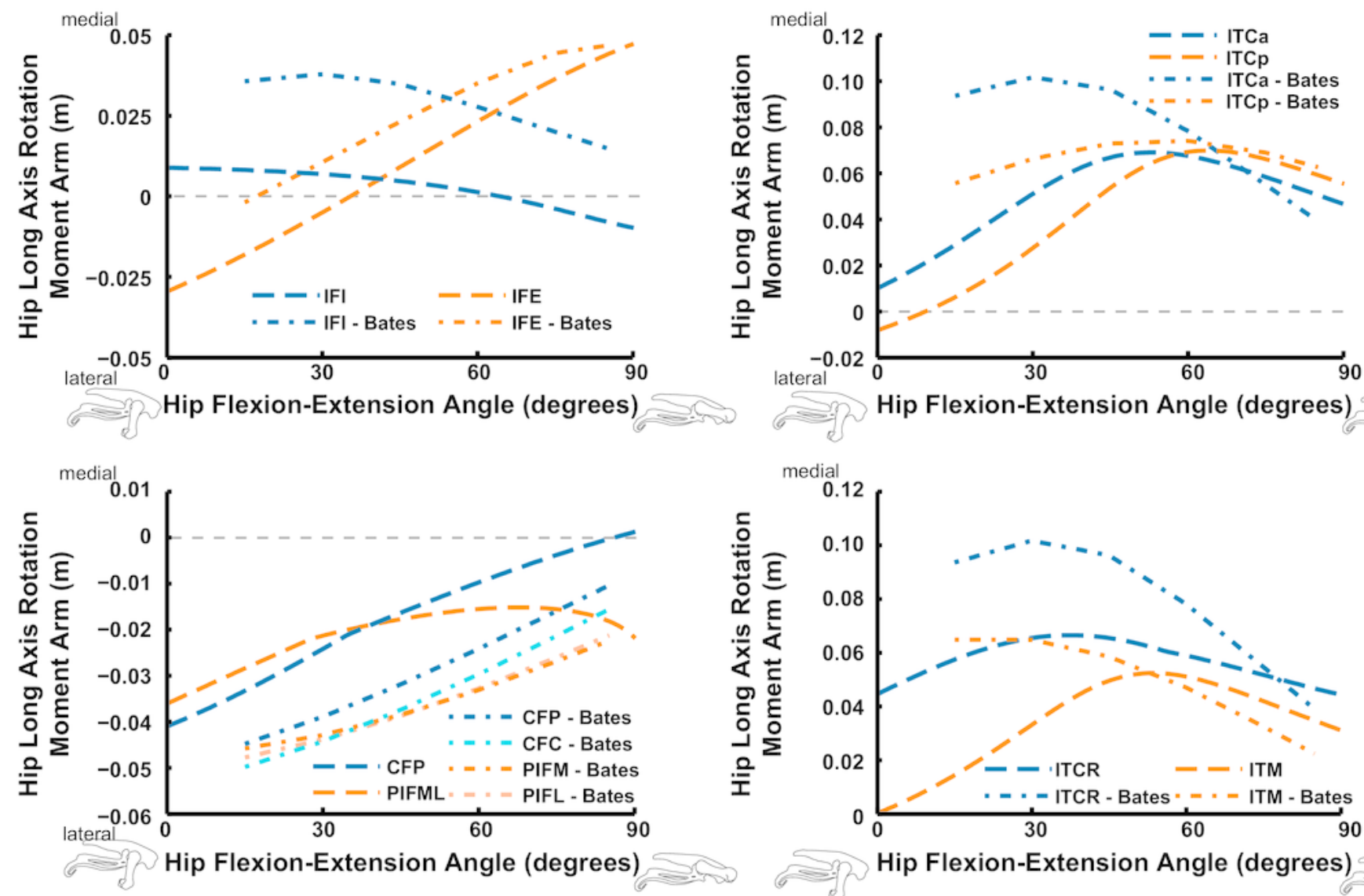
Hip Flexion-Extension Angle (degrees) 
Figure 14

Hip abduction/adduction moment arms plotted against hip flexion/extension joint angle for key proximal thigh muscles. See caption for Figure 9.
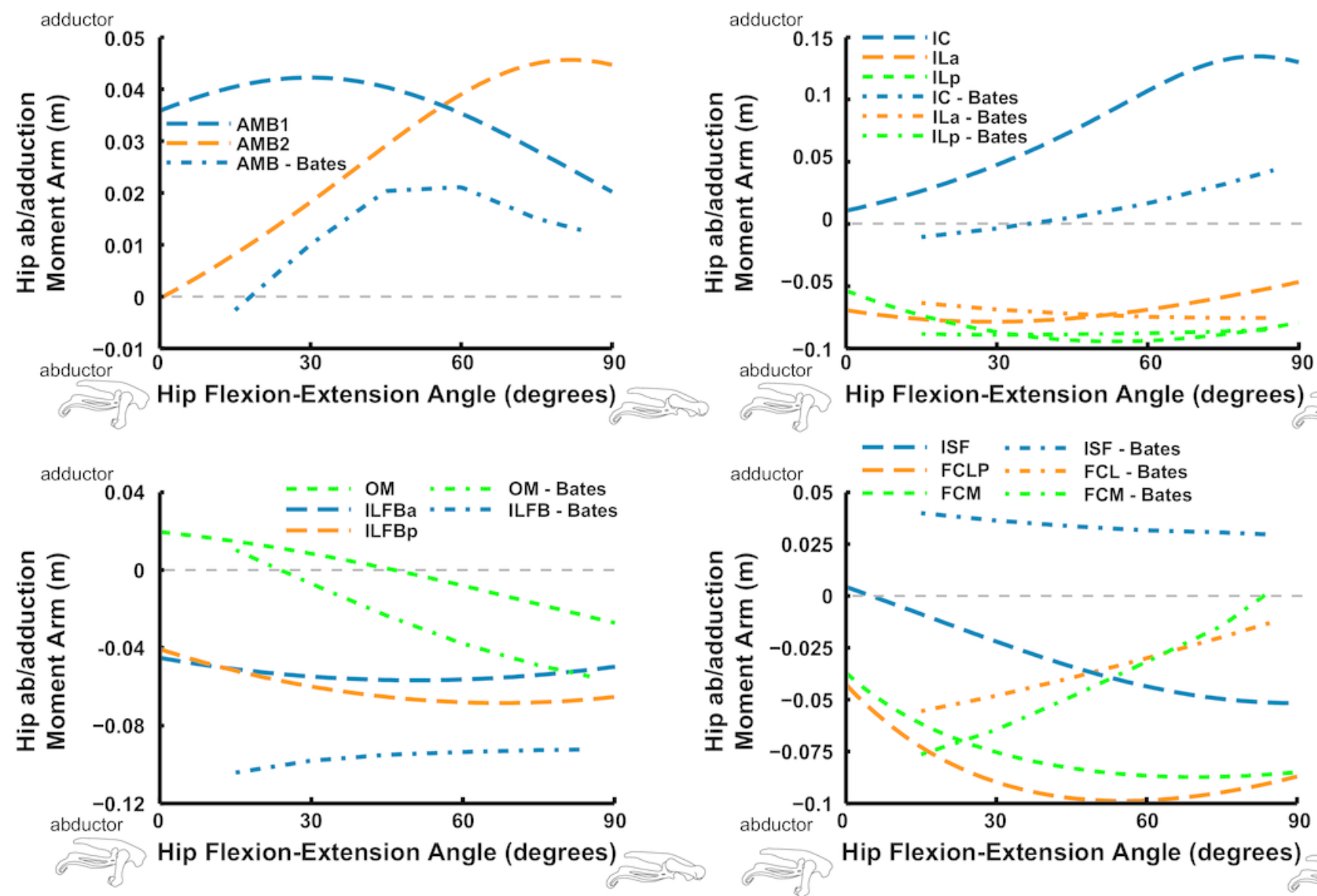

Hip Flexion-Extension Angle (degrees) 


\section{5}

\section{Figure 15}

Hip abduction/adduction moment arms plotted against hip flexion/extension joint angle for key proximal thigh muscles. See caption for Figure 9.
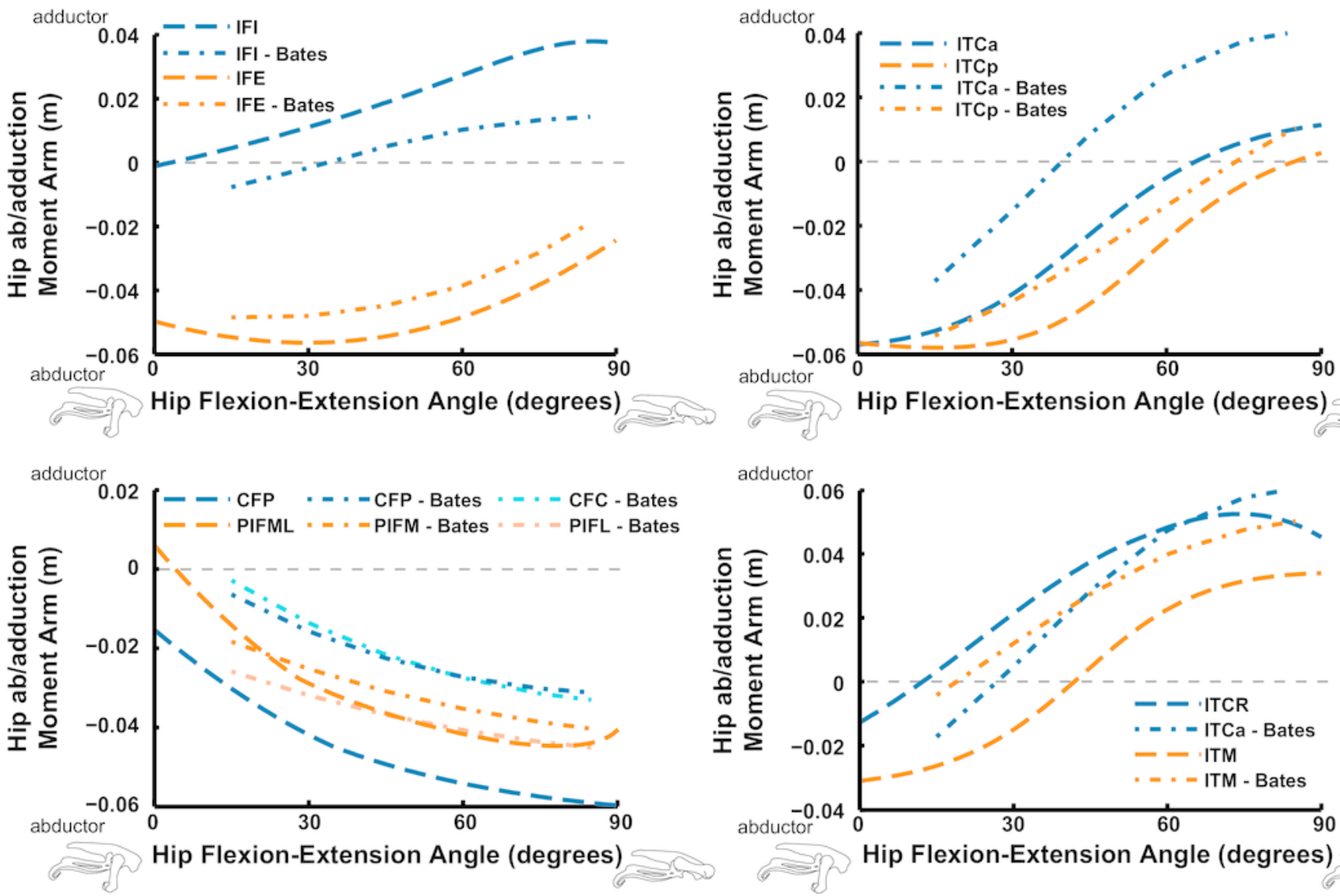


\section{6}

Figure 16

Knee flexor/extensor moment arms plotted against knee flexion/extension joint angle for key thigh muscles. See caption for Figure 9.
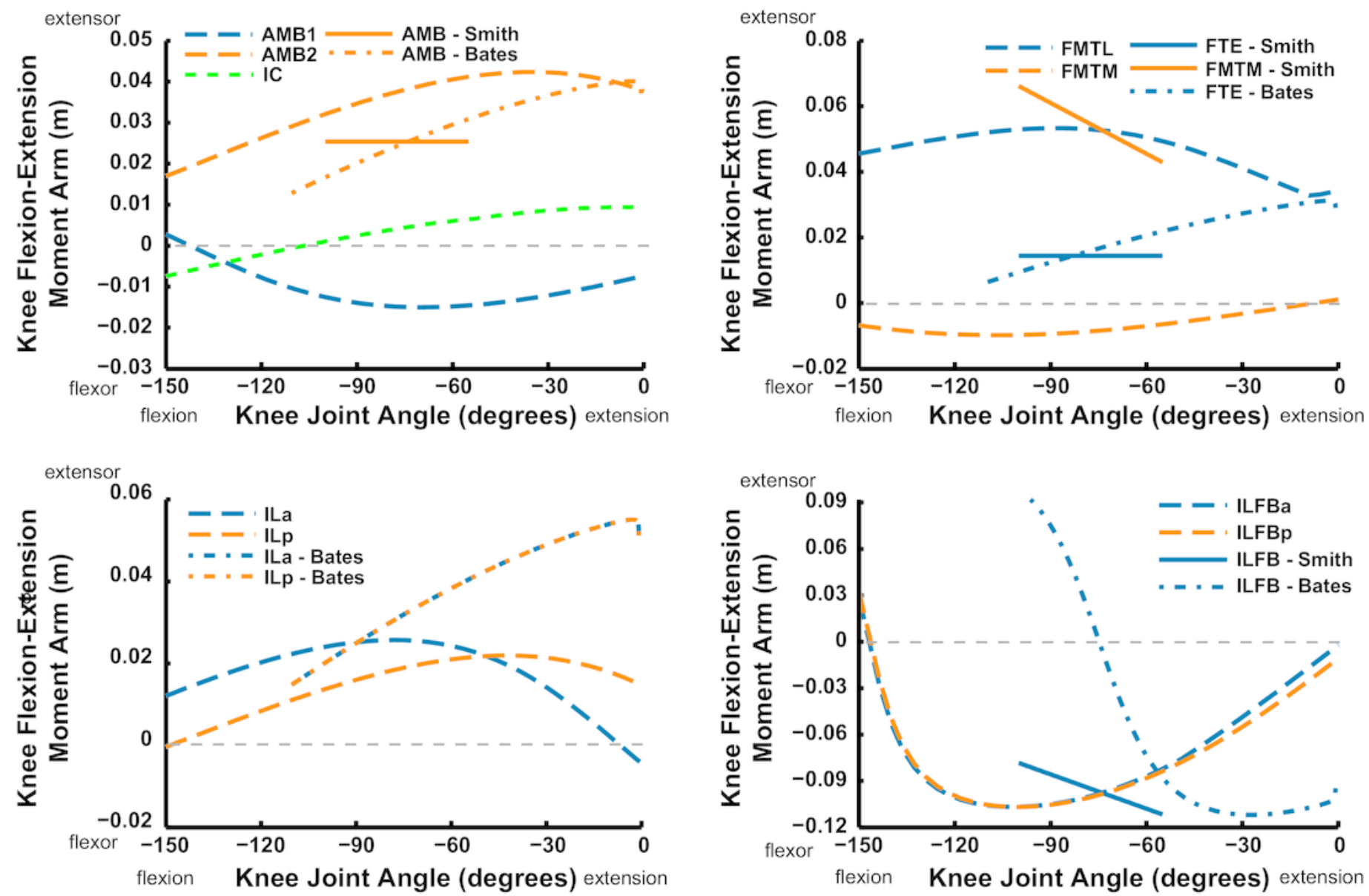
Figure 17

Knee flexor/extensor moment arms plotted against knee flexion/extension joint angle for key thigh and distal knee muscles. See caption for Figure 9.
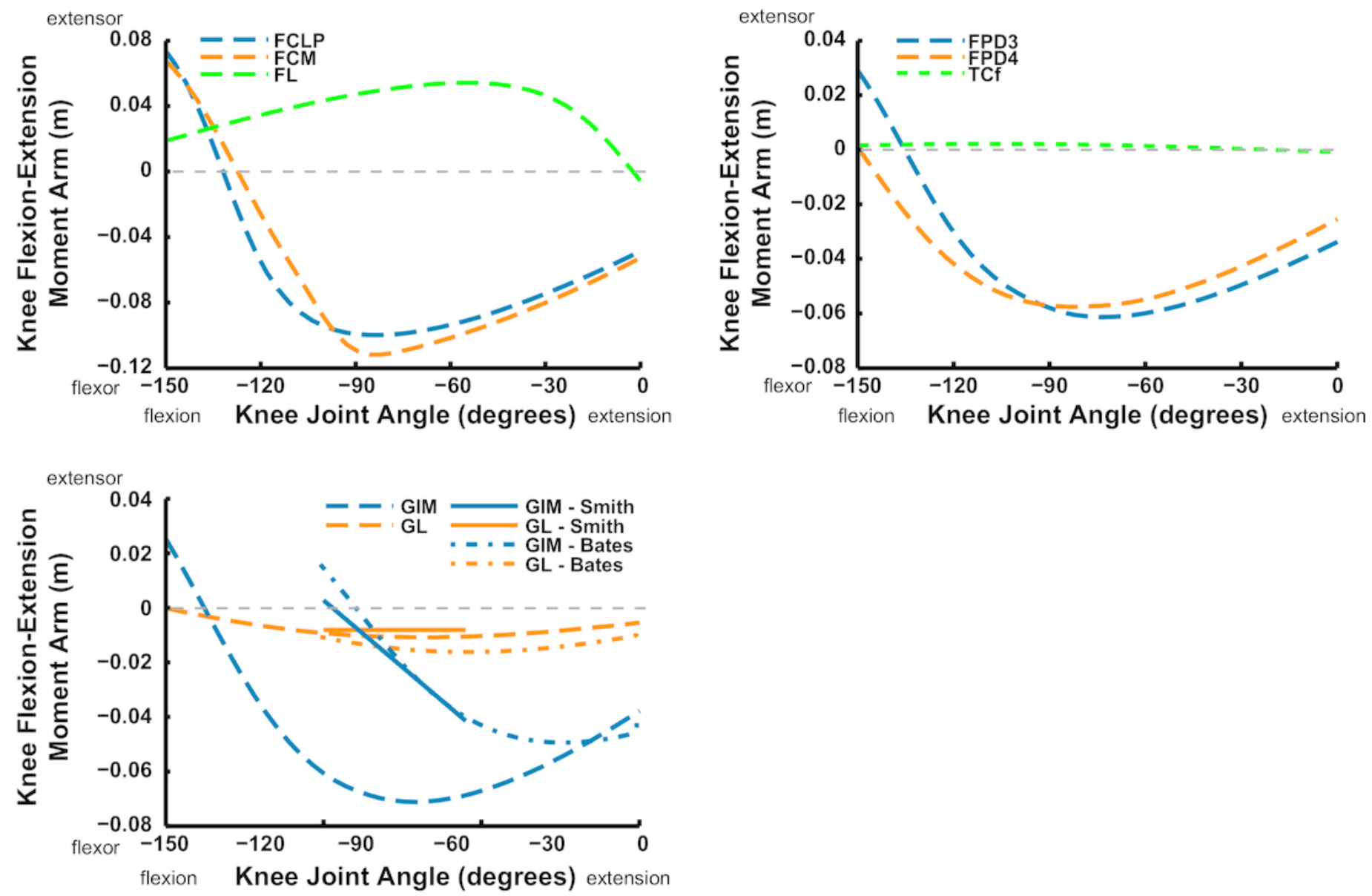


\section{8}

Figure 18

Ankle flexor/extensor moment arms plotted against ankle flexion/extension joint angle for key muscles crossing the ankle. See caption for Figure 9.
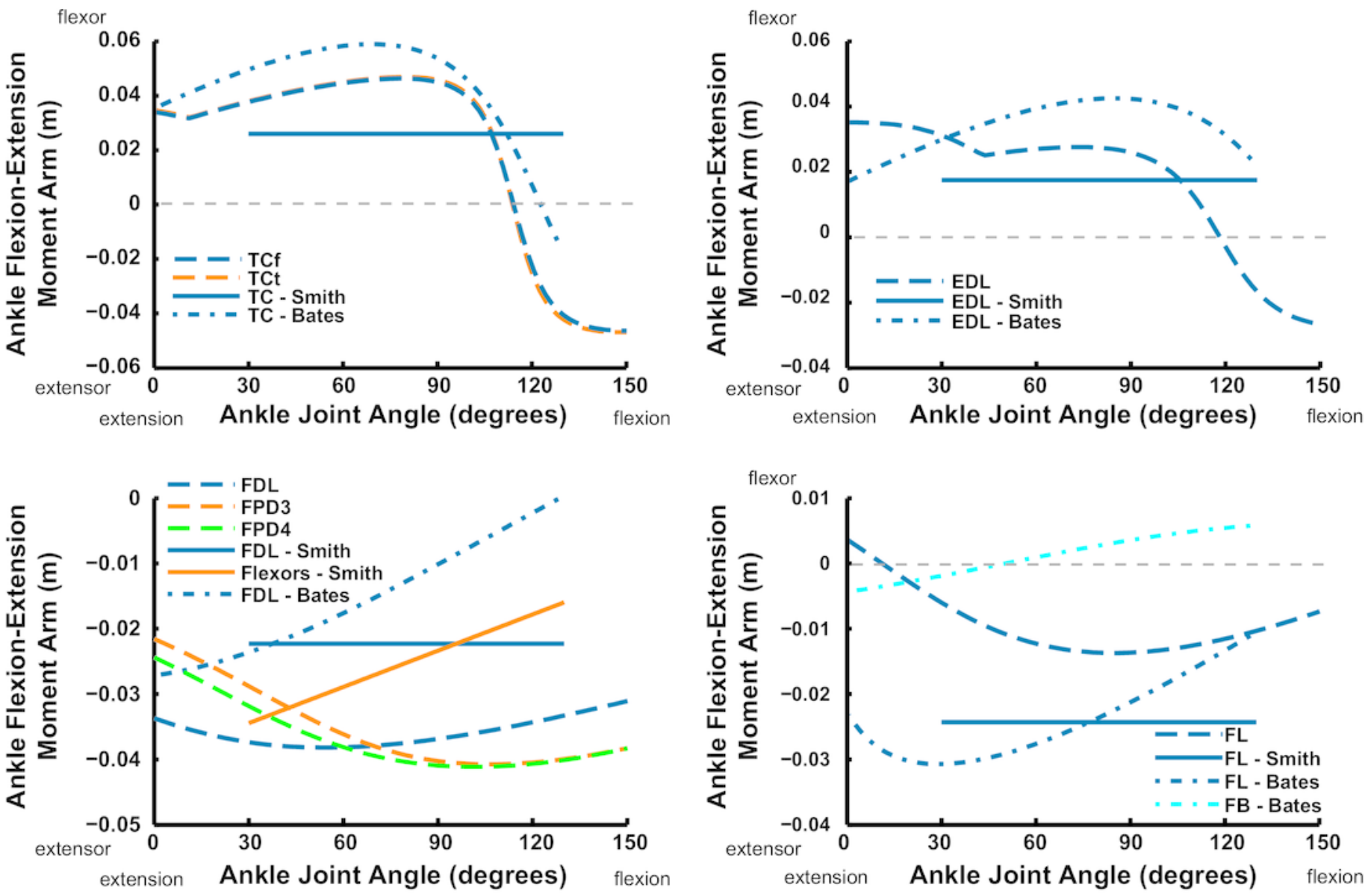


\section{9}

Figure 19

Ankle flexor/extensor moment arms plotted against ankle flexion/extension joint angle for the M. gastrocnemius muscle group. See caption for Figure 9.

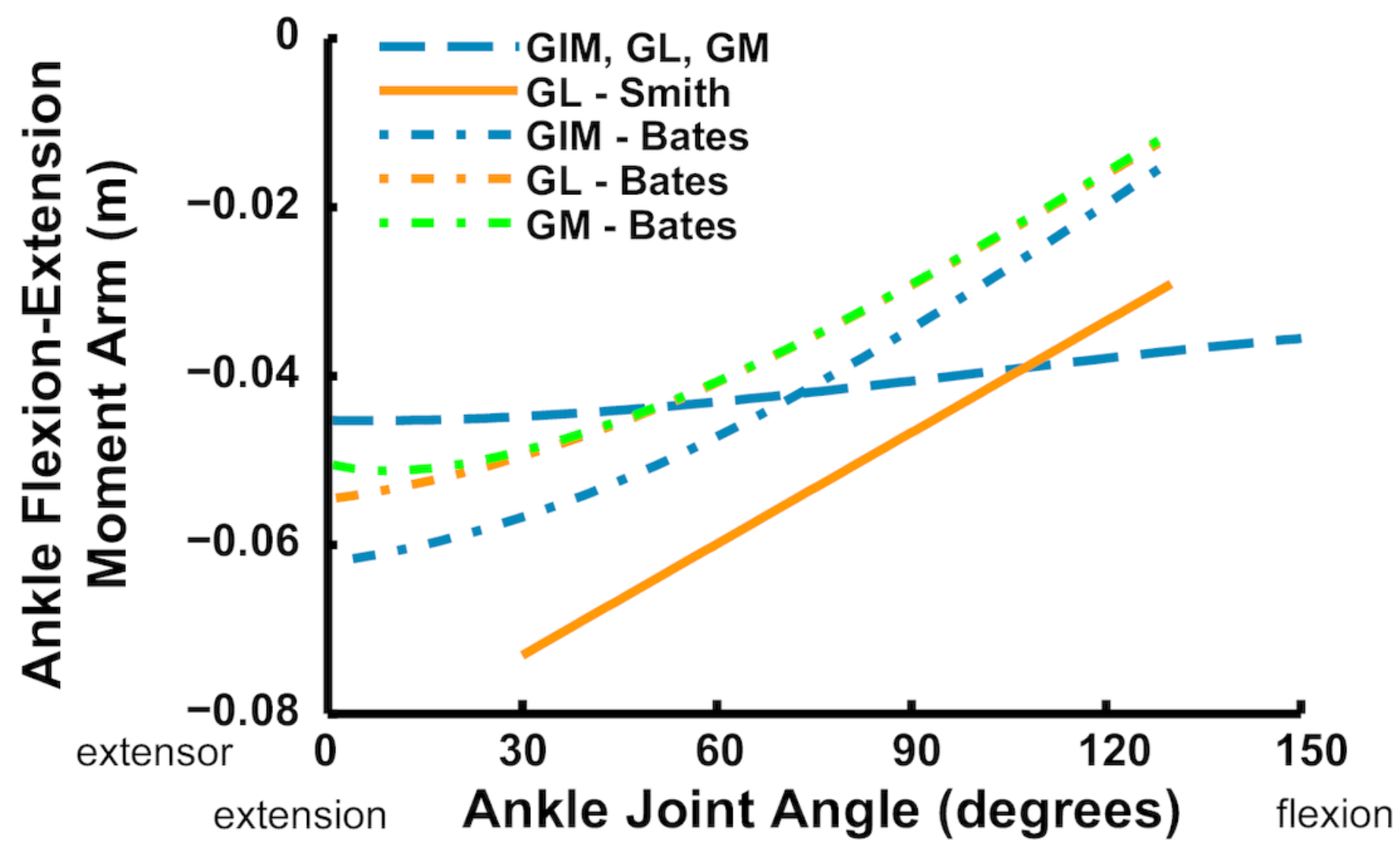


20

Figure 20

Metatarsophalangeal (MTP) joint flexor/extensor moment arms plotted against MTP flexion/extension joint angle for digital flexors (left) and extensors, plus tendinous connection of M. fibularis longus (right). See caption for Figure 9.
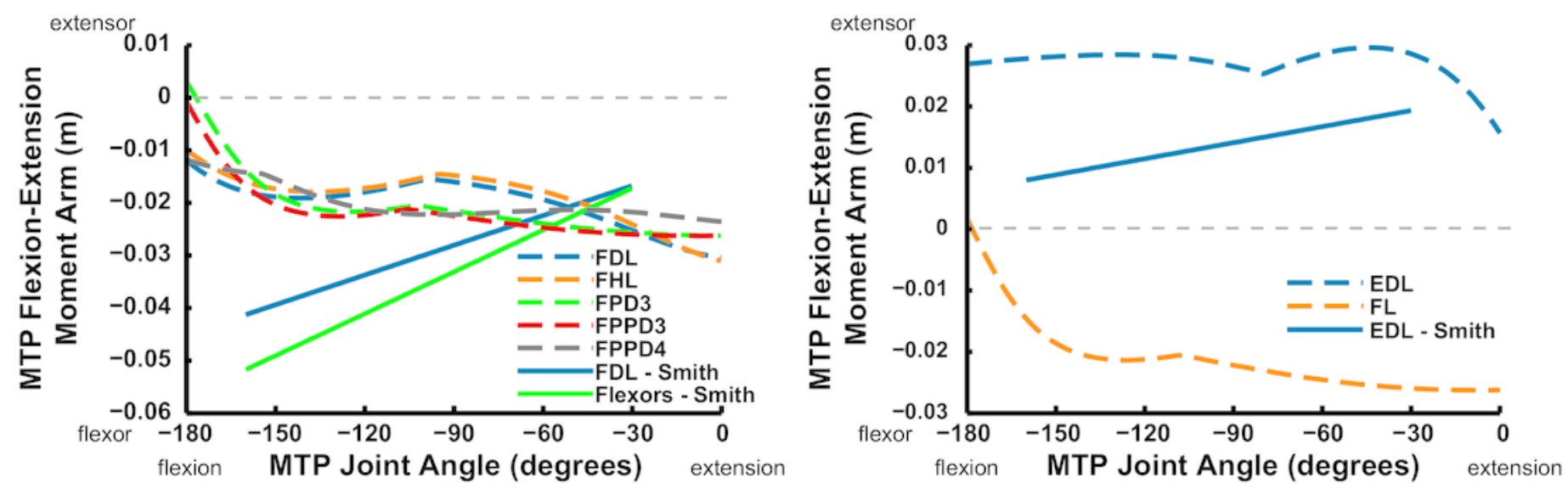